\title{
Auto-scaling Techniques for Clouds Processing Requests with Service Level Agreements
}

by

\author{
Anshuman Biswas
}

A thesis submitted to the Faculty of Graduate and Postdoctoral Affairs in partial fulfillment of the requirements for the degree of

\author{
Doctor of Philosophy \\ in
}

Electrical and Computer Engineering

Carleton University

Ottawa, Ontario

(C) 2019

Anshuman Biswas 


\section{Abstract}

Auto-scaling mechanisms allow applications running on Cloud environments to maintain a guaranteed Quality of Service while efficiently utilizing resources and keeping operational costs low for the service providers. However, creating such an auto-scaling framework may be challenging due to the need to precisely estimate resource usage while the workload patterns vary significantly.

The research presented in this thesis focuses on automatic provisioning of compute resources in the Cloud performed by an intermediary enterprise for a single client enterprise. The enterprise hosting a broker uses techniques for dynamically controlling the number of resources used by the client enterprise. The research introduces three autoscaling techniques: a reactive, a proactive and a hybrid technique. These techniques allow resources to be scaled based on user demand.

The primary goal of these auto-scaling techniques is to achieve a profit for the intermediary enterprise while maintaining the desired grade of service for the client enterprise. A secondary goal is to generate a lower cost for the client enterprise in comparison to the situation in which the client acquires resources directly from the cloud provider. The techniques support both on-demand requests as well as requests with service level agreements (SLAs). The effectiveness of the proposed auto-scaling techniques is demonstrated through experiments performed on proof of concept prototypes and simulations. The experimental results show that for a number of different combinations of system and workload parameters experimented with, the proposed algorithms lead to a significant broker profit and a lower user cost in comparison to a conventional non-autoscaling system. 


\section{Acknowledgments}

First and foremost, I would like to express my sincere gratitude to my thesis cosupervisors, Professor Shikharesh Majumdar and Professor Biswajit Nandy. Their passion for pushing the boundaries, in addition to their guidance, encouragement, and continuous support throughout the completion of my research and this thesis has left me forever indebted to them. I am greatly appreciative for the endless hours they've invested in me throughout my studies. This has not only shaped and guided my research but has also taught me the value of persistence and resilience in pursuing one's passion professionally. I would also like to thank Carleton University, the Government of Ontario, the Natural Science and Engineering Council (NSERC), and Telus Canada for providing financial support for this research. I would also like to acknowledge Ali El-Haraki from Telus Canada for his support towards this research.

Finally, I would like to thank my family, the most important people in my life who have always been there supporting me every step of the way. My wife, Tanya, has been a pillar of support throughout graduate school. Little did I know that my decision to travel halfway across the world to pursue a graduate degree would also lead me to find my life partner. Making the decision to spend the rest of my life with her is probably my greatest achievement throughout my graduate career. Without her by my side, cheering me on, completing this thesis would be hard to accomplish. From day one, my parents, Aloke and Sunita, have instilled the value of education, and the lifelong thirst of learning. I owe all that I am to them. I thank them for always encouraging me to complete this thesis, and their contribution cannot be captured just by this work alone. To my sister, Nayantara, I am indebted to her for always believing in me, and passing along her positive attitude, 
especially during those hard days. My heartfelt gratitude to my mother-in-law, Soma, for her unwavering support from the first day I met her. The teaching from my research extends well beyond what is published here. Graduate school has been a long, emotional, and at times challenging experience. However, it was worth every second that I spent on it, as I learned to appreciate that there truly are no limits to the what the mind can achieve. I hope this message comes through in parts of my research work. 


\section{Table of Contents}

Chapter 1 Introduction ................................................................................................1

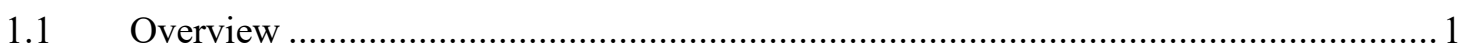

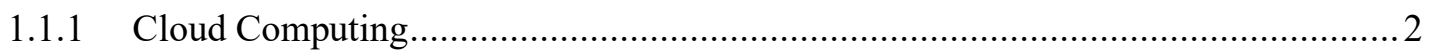

1.1.2 Service Level Agreements and QoS Guarantees ............................................. 3

1.1.3 Service Models .......................................................................................... 4

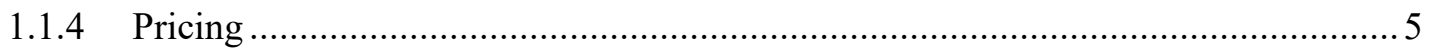

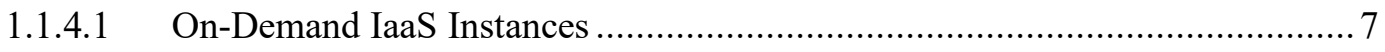

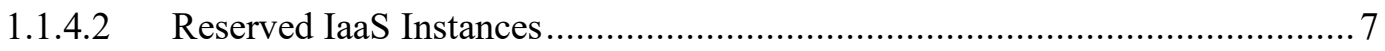

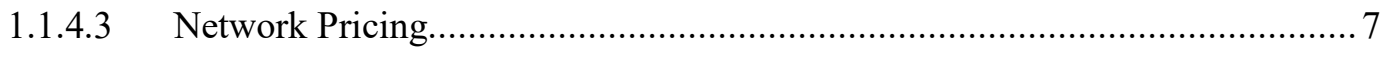

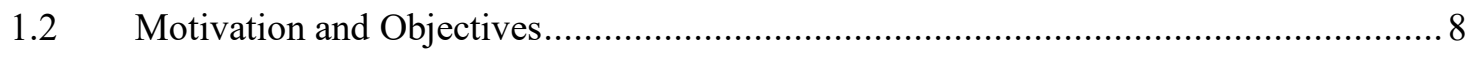

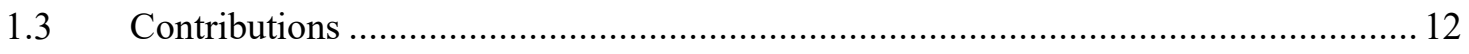

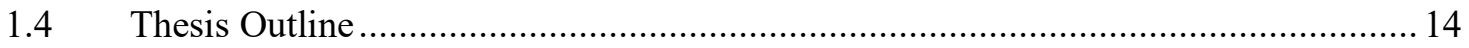

Chapter 2 Related Work..................................................................................16

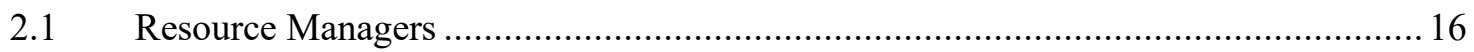

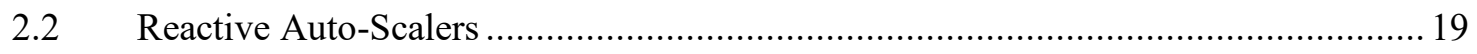

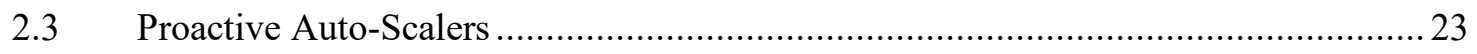

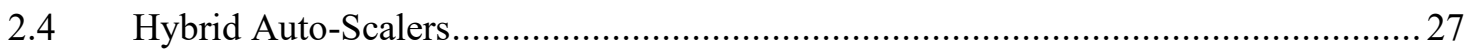

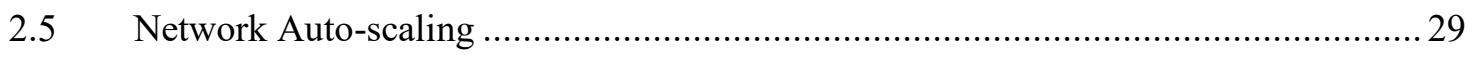

Chapter 3 The Auto-scaling System ........................................................................34

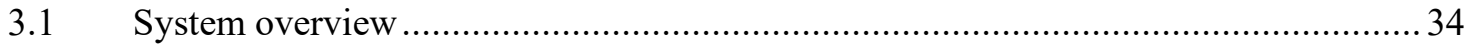

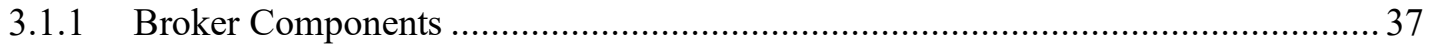

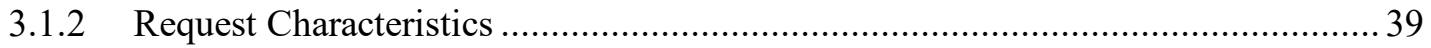

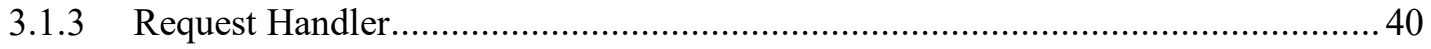

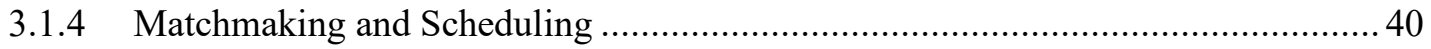




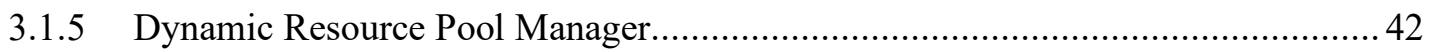

3.2 Broker Pricing Model .................................................................................. 43

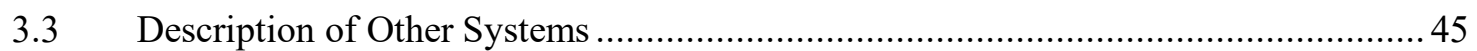

\section{Chapter 4 The Reactive Auto-scaling Technique ....................................................47}

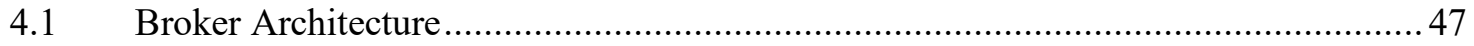

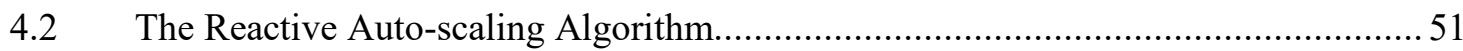

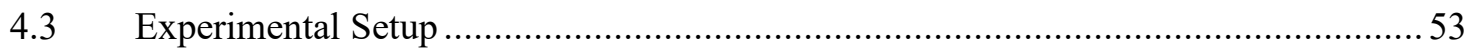

4.3.1 Programming Language/Framework.......................................................... 53

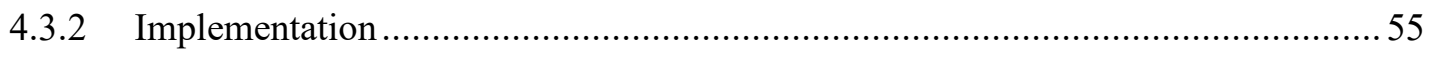

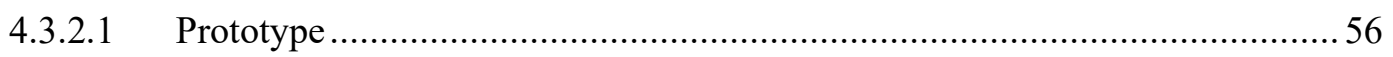

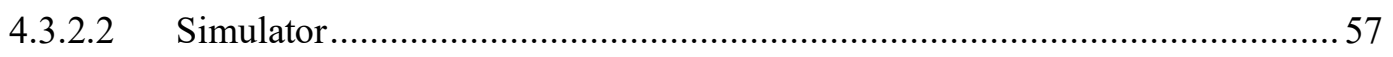

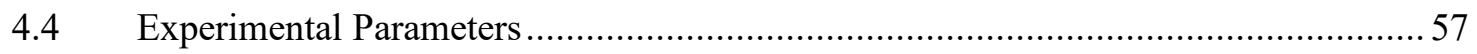

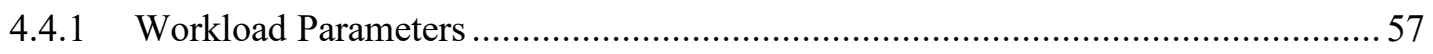

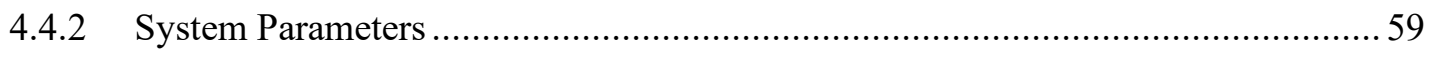

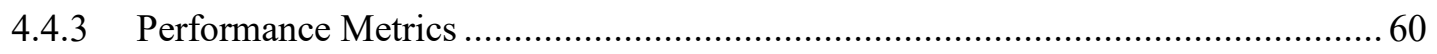

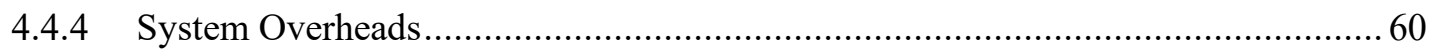

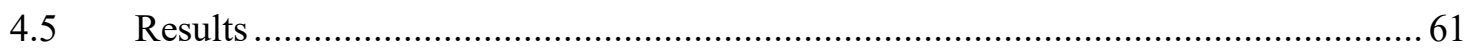

4.5.1 Demonstration of Auto-scaling Performed on the System .................................. 62

4.5.2 Comparison between the Simulator and the Prototype ......................................63

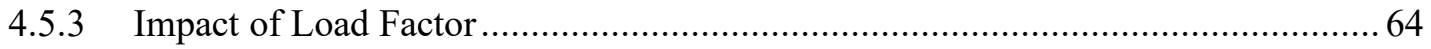

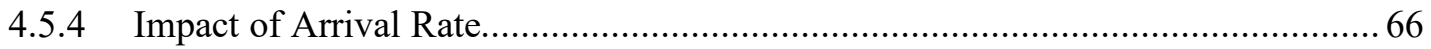

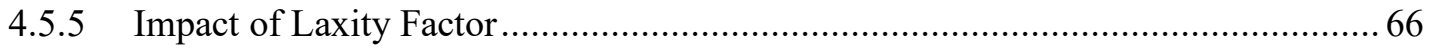

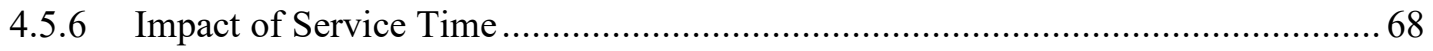

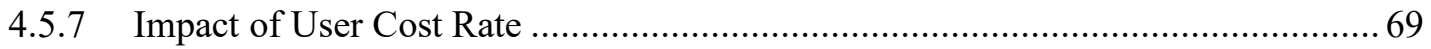

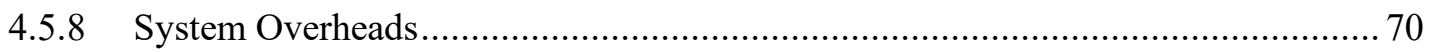

4.5.9 Impact of Other factors on Total User Cost................................................ 70 
4.6 Observations

\section{Chapter 5 The Proactive Auto-scaling Technique..............................................74}

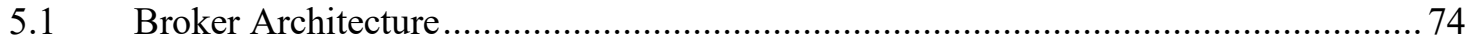

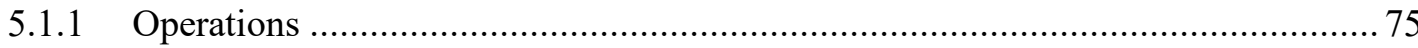

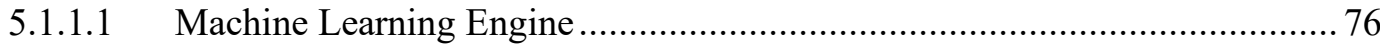

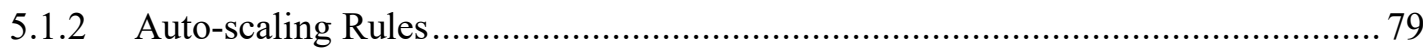

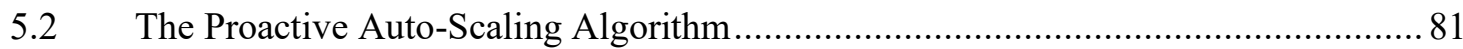

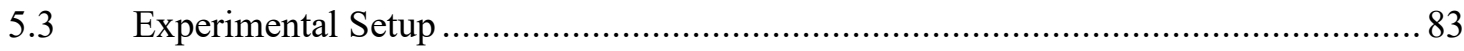

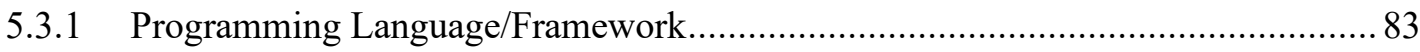

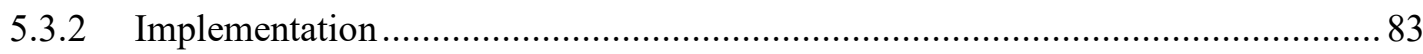

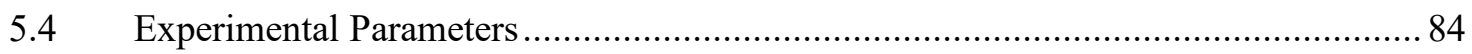

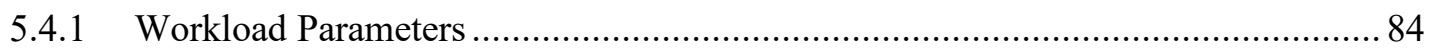

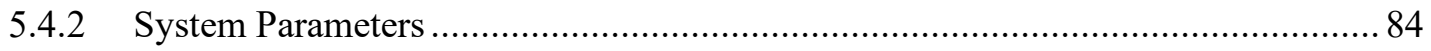

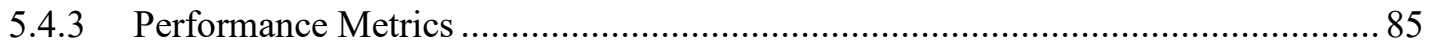

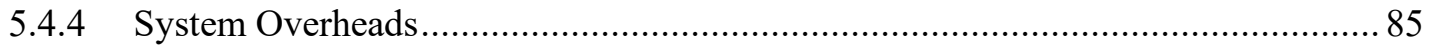

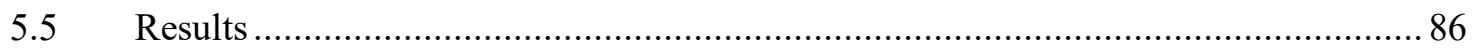

5.5.1 Comparison between the Simulator and the Prototype …................................... 86

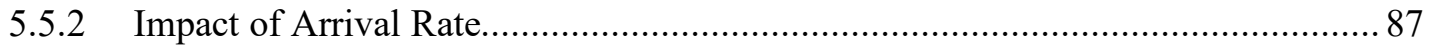

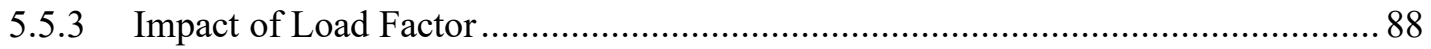

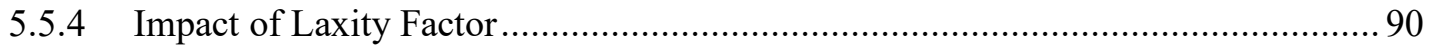

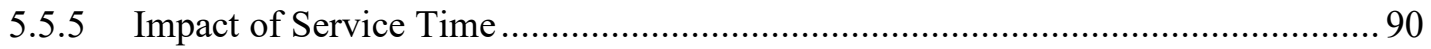

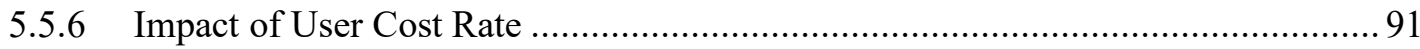

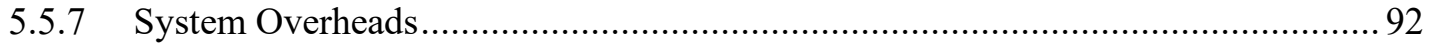

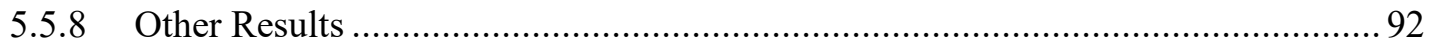

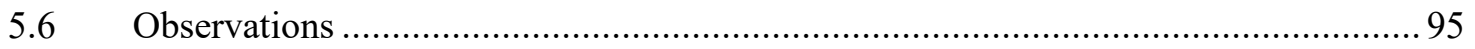


Chapter 6 The Hybrid Auto-scaling Technique.....................................................97

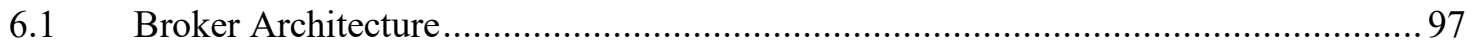

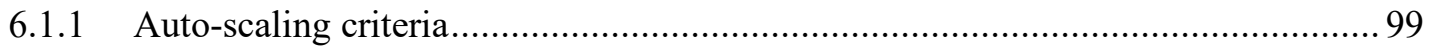

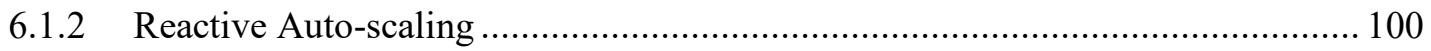

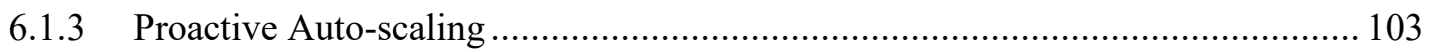

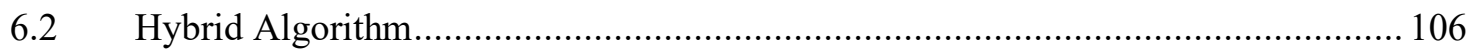

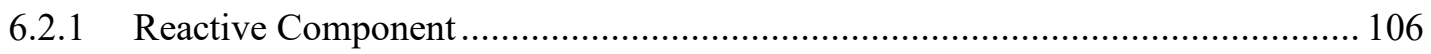

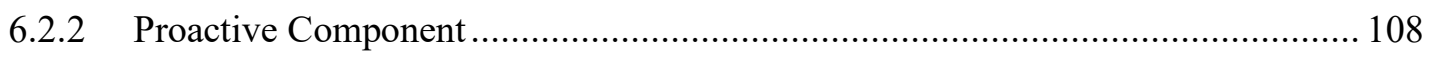

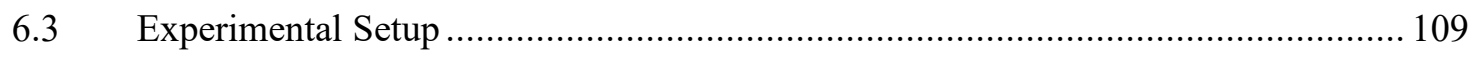

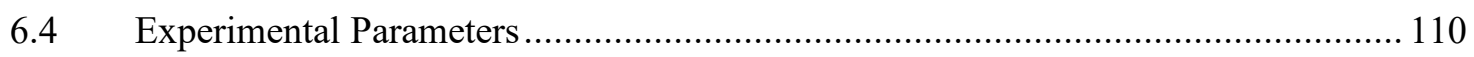

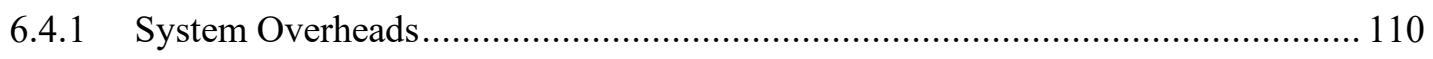

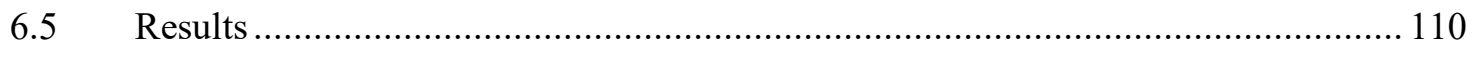

6.5.1 Comparison between the Simulator and the Prototype ................................... 111

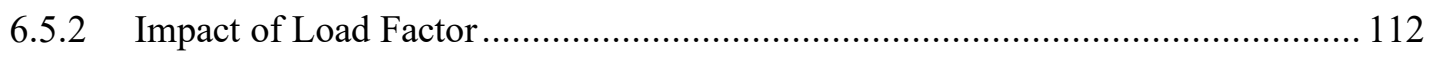

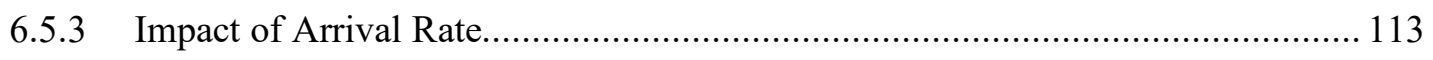

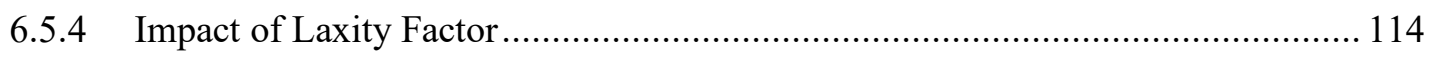

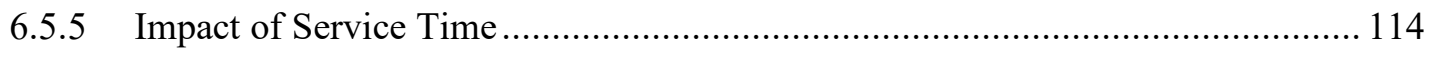

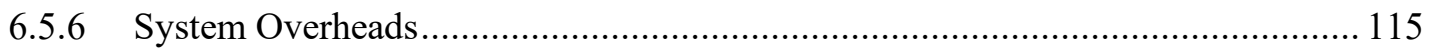

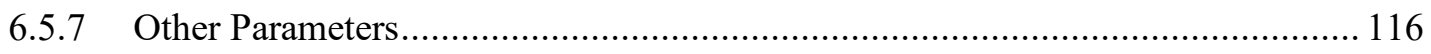

6.5.7.1 Impact of Number of Requests between Predictions ............................... 116

6.5.7.2 Impact of Machine Learning Algorithm ............................................ 117

6.5.8 Comparison with Pure Reactive and Pure Proactive Approaches........................ 117

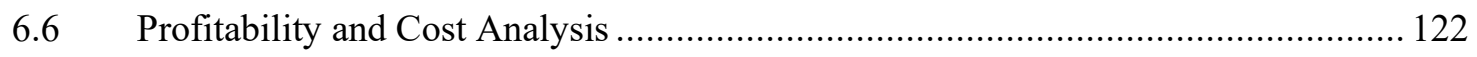

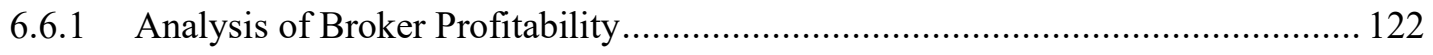

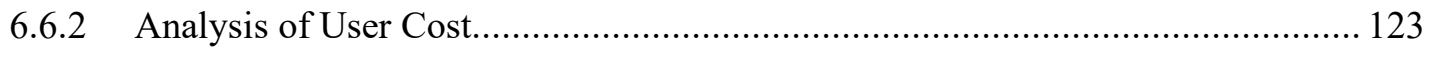

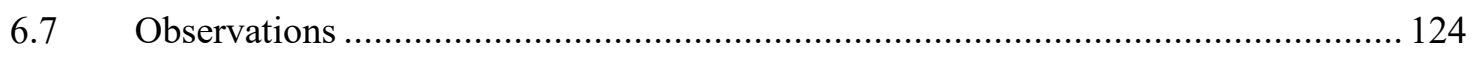




\section{Chapter 7 Extending the Auto-Scaling Approach to Network Resources .........126}

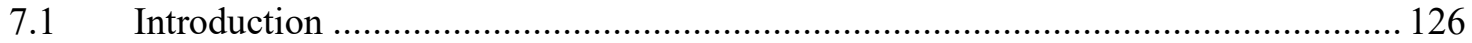

7.1.1 Supporting Technology for enabling auto-scaling network resources ................. 129

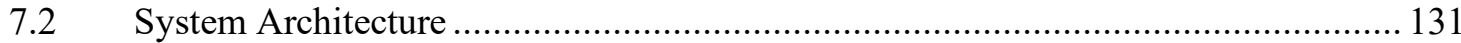

7.2.1 Request Handling and Updated Pricing ........................................................ 133

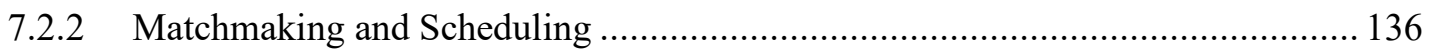

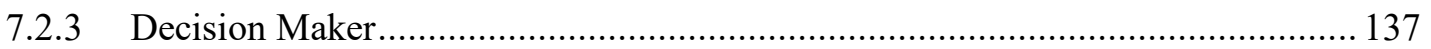

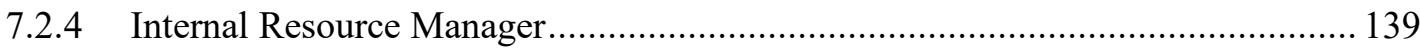

7.3 The Auto-scaling Algorithm with Network Resources .......................................... 139

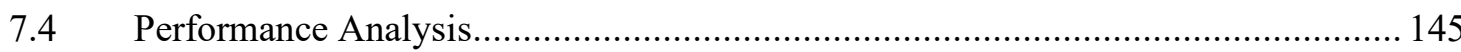

7.4.1 Experimental Parameters.......................................................................... 145

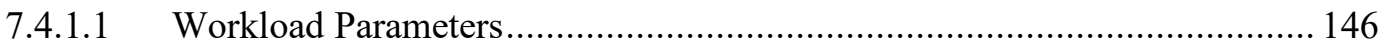

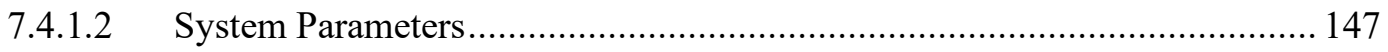

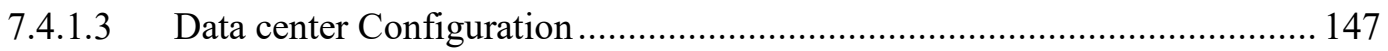

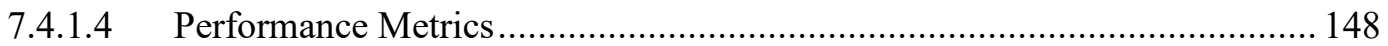

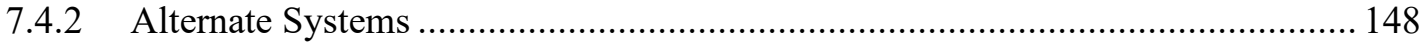

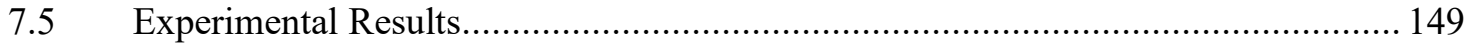

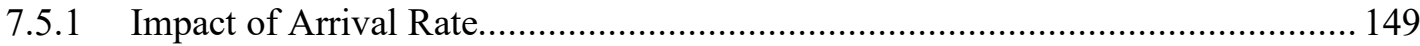

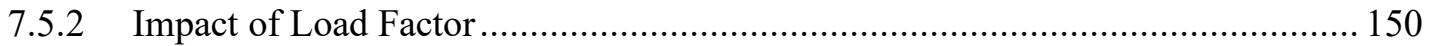

7.5.3 Measure of East-West Traffic .............................................................................. 151

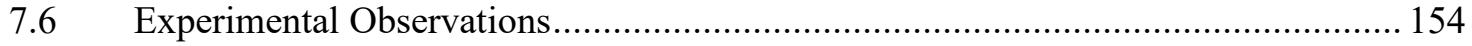

Chapter 8 Summary and Conclusions.....................................................................157

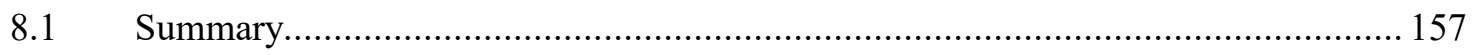

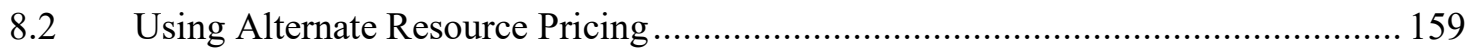

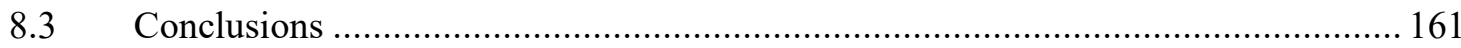

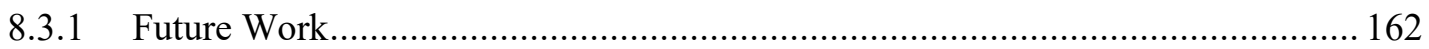




\section{List of Tables}

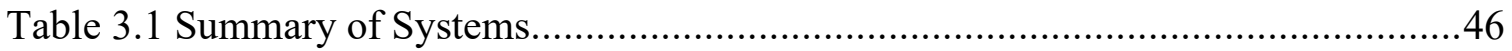

Table 4.1 Reactive Auto-Scaling Algorithm............................................................52

Table 4.2 Summary of Workload Parameters for Reactive System .................................59

Table 4.3 Summary of System Parameters for Reactive System....................................59

Table 4.4 Highest Percentage Cost Savings of System I-R Over System II and System III

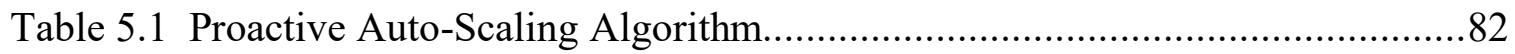

Table 5.2: Summary of System Parameters for Proactive System..................................85

Table 5.3: Average Percentage Error in Prediction using LR and SVR..........................94

Table 6.1 Hybrid Auto-Scaling Algorithm: Part 1 ................................................... 107

Table 6.2 Hybrid Auto-Scaling Algorithm: Part 2a ................................................... 109

Table 6.3 Hybrid Auto-Scaling Algorithm: Part 2b (SelectResource) ...........................109

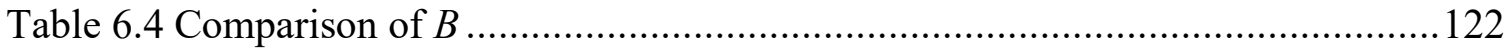

Table 7.1 Auto-Scaling Algorithm with Network Resources ......................................144

Table 7.2 Summary of Workload Parameters ..............................................................146

Table 7.3 Summary of System Parameters ………….............................................147

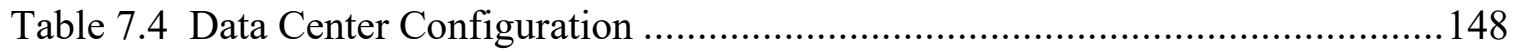




\section{List of Figures}

Figure 1.1: Service models for Cloud Computing [11] .......................................

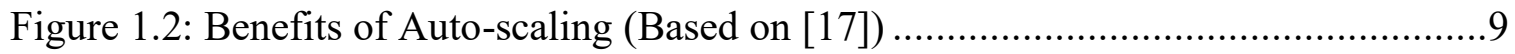

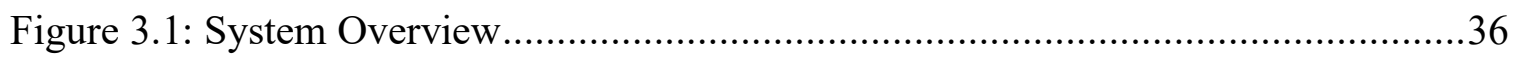

Figure 3.2: Broker Architecture .......................................................................... 38

Figure 4.1: Reactive Broker Architecture ..............................................................48

Figure 4.2: Relationship between the Number of Resources and Number of Active

Requests for the Reactive System .................................................................6

Figure 4.3: Comparison of the Simulator and Prototype for the Reactive Auto-scaler.....64

Figure 4.4: Effect of $f$ on Broker Profit for the Reactive Auto-scaler ..........................65

Figure 4.5: Effect of $f$ on User Cost for the Reactive Auto-scaler ..............................66

Figure 4.6: Effect of $\lambda$ on Broker Profit for the Reactive Auto-scaler ..........................67

Figure 4.7: Effect of $L f$ on Broker Profit for the Reactive Auto-scaler ........................67

Figure 4.8: Effect of $S$ on Broker Profit for the Reactive Auto-scaler ........................69

Figure 4.9: Effect of $c \_p v t$ on Broker Profit for the Reactive Auto-scaler..................... 70

Figure 5.1: Proactive Broker Architecture ..................................................... 75

Figure 5.2: Proactive Broker: Collaboration Diagram …...................................... 76

Figure 5.3: Proactive Broker: Sequence Diagram............................................ 78

Figure 5.4: Comparison of the Simulator and Prototype for the Proactive Auto-scaler ...88

Figure 5.5: Effect of $\lambda$ on Broker Profit for the Proactive System ...............................8 88

Figure 5.6: Effect of $f$ on Broker Profit for the Proactive System ................................89

Figure 5.7: Effect of $f$ on User Cost for the Proactive System..................................90

Figure 5.8: Effect of $L f$ on Broker Profit for the Proactive System .............................91 
Figure 5.9: Effect of $S$ on Broker Profit for the Proactive System..............................92

Figure 5.10 Effect of $c \_p v t$ on Broker Profit for the Proactive System ........................93

Figure 5.11: Effect of $P$ on Broker Profit for the Proactive System .............................93

Figure 5.12: Effect of changing the $M L A$ on Broker Profit for the Proactive System ......94

Figure 6.1: Hybrid Broker Architecture ...........................................................98

Figure 6.2: Comparison of the Simulator and Prototype for the Hybrid Auto-scaler ..... 112

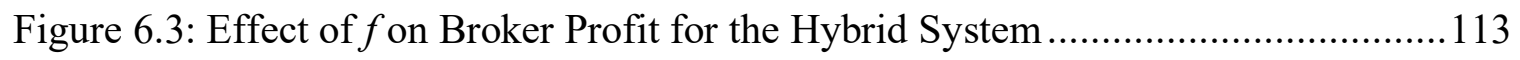

Figure 6.4: Effect of $f$ on User Cost for the Hybrid System .................................. 113

Figure 6.5: Effect of $\lambda$ on Broker Profit for the Hybrid System............................. 115

Figure 6.6: Effect of $L f$ on Broker Profit for the Hybrid System ..............................115

Figure 6.7: Effect of $S$ on Broker Profit for the Hybrid System ................................116

Figure 6.8: Effect of $P$ on Broker Profit for the Hybrid System.............................. 117

Figure 6.9: Effect of changing the MLA on Broker Profit for the Hybrid System......... 118

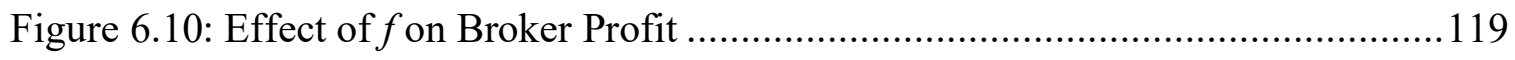

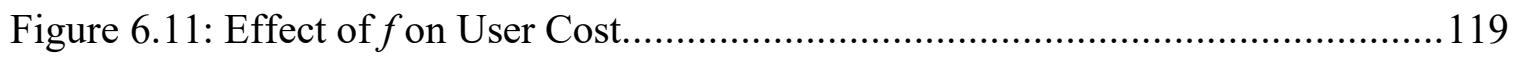

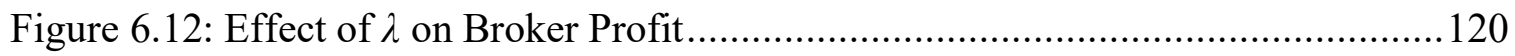

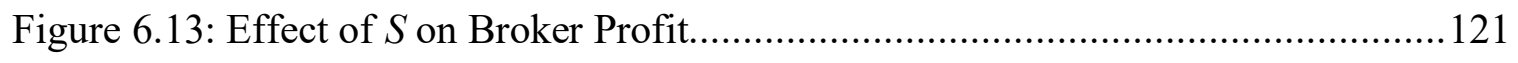

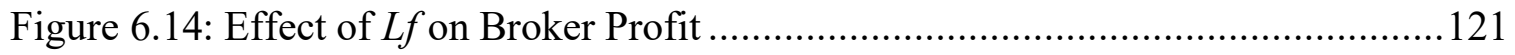

Figure 7.1: Traditional Data Center Topology..................................................... 129

Figure 7.2: Broker Architecture for Handling Network Resources ........................... 133

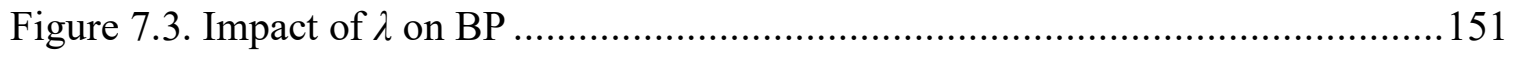

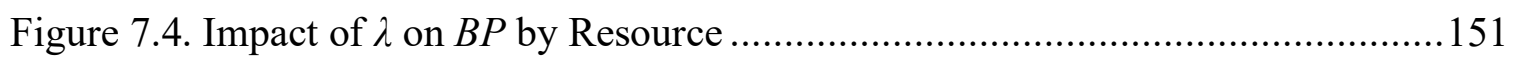

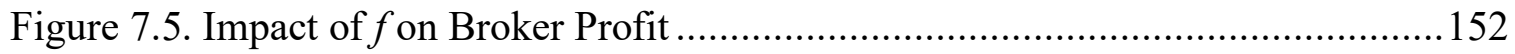




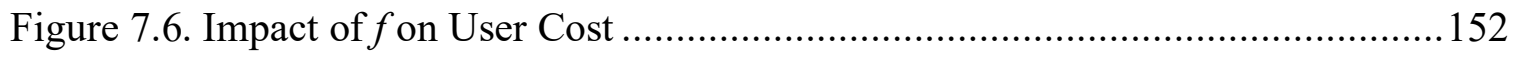

Figure 7.7. Comparison of Match-making Algorithms ......................................... 153

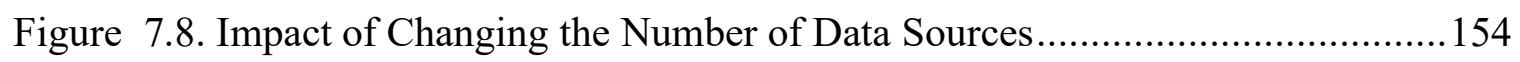




\section{List of Symbols}

\begin{tabular}{|c|c|}
\hline$B C^{p_{i}}$ & Predicted Broker Cost for the $\mathrm{i}^{\text {th }}$ request \\
\hline$B^{p}$ & Predicted Probability of Blocking \\
\hline$B P^{a}$ & Actual Broker Profit \\
\hline$B P_{i}$ & The profit accrued by the broker for servicing the $\mathrm{i}^{\text {th }}$ request \\
\hline$B P^{p}{ }_{i}$ & Predicted Broker Profit for the $\mathrm{i}^{\text {th }}$ request \\
\hline$B_{Q}$ & Reserved Bandwidth of network link Q \\
\hline$B_{\text {spec }}$ & Specified Probability of Blocking \\
\hline c_pub/c_pub ${ }_{\text {compute }}$ & User cost rate for the compute resource \\
\hline c_pub $b_{\text {network }}$ & User cost rate for the network resource \\
\hline c_pvt/c_pvt compute & Broker cost rate for the compute resource \\
\hline c_pvt $t_{\text {network }}$ & Broker cost rate for the network resource \\
\hline$c_{i}$ & Service Time for the $\mathrm{i}^{\text {th }}$ request \\
\hline$d_{k}$ & Total data transferred for $\mathrm{k}$ requests \\
\hline$D S I_{i}$ & Set of input data stores for the $\mathrm{i}^{\text {th }}$ request \\
\hline $\mathrm{DSO}_{i}$ & Output data store for the $\mathrm{i}^{\text {th }}$ request \\
\hline$f$ & Load Factor \\
\hline G & Constant used by each auto-scaler \\
\hline$L f$ & Laxity Factor \\
\hline$l_{k j}$ & $\mathrm{j}$ link demands for $\mathrm{k}$ requests \\
\hline$n d_{j}$ & Network demand for the $j^{\text {th }}$ link \\
\hline $\operatorname{Res}_{N}$ & $\mathrm{~N}$ number of resources controlled by the broker \\
\hline$S$ & Mean Ser \\
\hline
\end{tabular}




$\begin{array}{ll}s_{i} & \text { Predicted Service Time for the } \mathrm{i}^{\text {th }} \text { request } \\ t_{r} & \text { Type of Request } \\ T_{r} & \text { Time for which compute resource } \mathrm{r} \text { is executing requests } \\ U C^{p}{ }_{i} & \text { Time for which the compute resource } \mathrm{r} \text { has been rented } \\ U_{r} & \text { Predicted Total User Cost for the } \mathrm{i}^{\text {th }} \text { request } \\ \lambda & \text { Mean Arrival Rate }\end{array}$




\section{List of Acronyms}

\begin{tabular}{|c|c|}
\hline API & Application Programming Interface \\
\hline $\mathrm{AR}$ & Advance Reservation \\
\hline $\mathrm{AR}$ & Aggregation Router \\
\hline ARMA & Auto-regressive Moving Average \\
\hline AS & Aggregation Switch \\
\hline AT & Arrival Time \\
\hline AWS & Amazon Web Services \\
\hline $\mathrm{BC}$ & Broker Cost \\
\hline $\mathrm{BF}$ & Best Fit \\
\hline BP & Broker Profit \\
\hline CAPEX & Capital Expenditure \\
\hline $\mathrm{CR}$ & Core Router \\
\hline $\mathrm{CS}$ & Total Cost Savings \\
\hline $\mathrm{DC}$ & Data Center \\
\hline $\mathrm{DCN}$ & Data Center Network \\
\hline DL & Deadline \\
\hline $\mathrm{DM}$ & Decision Maker \\
\hline DRPM & Dynamic Resource Pool Manager \\
\hline DS & Data Store \\
\hline DU & Data Center Network Utilization \\
\hline EDF & Earliest Deadline First \\
\hline ES & Earliest Start Time \\
\hline
\end{tabular}




$\begin{array}{ll}\text { FF } & \text { First Fit } \\ \text { GoS } & \text { Grade of Service } \\ \text { IaaS } & \text { Infrastructure as a Service } \\ \text { IE } & \text { Intermediary Enterprise } \\ \text { IRM } & \text { Internal Resource Manager } \\ \text { JSON } & \text { JavaScript Object Notation } \\ \text { LR } & \text { Linear Regression } \\ \text { MLA } & \text { Machine Learning Algorithm } \\ \text { MLE } & \text { Machine Learning Engine } \\ \text { MMS } & \text { Matchmaking and Scheduling } \\ \text { ND } & \text { Network Demand } \\ \text { NIST } & \text { National Institute of Standards and Technology } \\ \text { NN } & \text { Neural Networks } \\ \text { OD } & \text { On-demand } \\ \text { OPEX } & \text { Operational Expenditure } \\ \text { PaaS } & \text { Platform as a Service } \\ \text { QoS } & \text { Quality of Service } \\ \text { RA } & \text { Reactive Auto-scaler } \\ \text { REST } & \text { Representational State Transfer } \\ \text { RH } & \text { Request Handler } \\ \text { SaaS } & \text { Software as a Service } \\ \text { SCE } & \text { Singlenterprise } \\ \text { SDelopment Kit }\end{array}$




$\begin{array}{ll}\text { SLA } & \text { Service Level Agreement } \\ \text { SST } & \text { Scheduled Start Time } \\ \text { SVR } & \text { Support Vector Regression } \\ \text { TAG } & \text { Tenant Application Graph } \\ \text { TCP } & \text { Transmission Control Protocol } \\ \text { ToR } & \text { Top-of-Rack } \\ \text { TUC } & \text { Total User Cost } \\ \text { VDC } & \text { Virtual Data Center } \\ \text { VM } & \text { Virtual Machine } \\ \text { VN } & \text { Virtual Network } \\ \text { VPC } & \text { Virtual Private Cloud } \\ \text { XML } & \text { Extensible Markup Language }\end{array}$




\section{Chapter 1 Introduction}

The cloud computing paradigm has become pervasive because of an increase of applications deployed on configurable resources (e.g., networks, servers, storage, applications, and services) that can be rapidly provisioned. The cloud environment offers an organization the ability to shift its IT operations from a traditional Capital Expenditure (CAPEX) model [1], where the organization procures dedicated hardware that depreciates over time to an Operational Expenditure (OPEX) model. This allows the use of a shared cloud infrastructure on-demand, with the benefit of only paying for the amount of resources used up by the organization. This flexible pricing scheme offers the ability for cloud users to acquire resources by paying a fixed price per unit time for a cloud resource, also known as an on-demand or a pay-as-you-go pricing model [2]. This chapter presents an overview of cloud computing leading up to the necessity to introduce auto-scaling, and subsequently highlighting the benefits of employing auto-scaling techniques in cloud computing environments. Several use cases that establish the relevance of the auto-scaling problem are presented. The chapter also describes the challenges faced while employing autoscaling strategies. Finally, the chapter concludes with a summary of the important contributions of the thesis.

\subsection{Overview}

Cloud computing has become a mainstream part of the Information Technology (IT) world, with far-reaching impacts for many enterprises. The cloud offers a solution for capacity planning issues that have plagued organizations and IT departments in the past. Capacity planning is the ability for estimating the computer hardware, software and connection infrastructure resources that will be demanded by the various applications run 
by the enterprise, over a period of time. This application demand, also known as workload demand [3], is the demand placed on a system resources, such as CPU, memory, I/O throughput and network throughput, by the enterprise's application. More enterprises are looking to migrate their workloads running on-premises systems to an equivalent cloudprovided platform. To realize this business opportunity, Gartner predicts [4] that the number of cloud managed service providers is predicted to triple by 2020 while the cloud computing services will be a $\$ 300$ billion business by 2021 . While enterprises are keen on migrating their applications to expand into cloud services and making them an integral part of their digital business initiatives, three major concerns need to be addressed, according to research firm, International Data Corporation [5]. The first two are based on the perceived risks of unauthorized access to sensitive or restricted information, and the possibility that the cloud provider may not be able to provide access to information indefinitely as a result of disruption in operations. These are concerns that the enterprises have based on the reputation and trust of a cloud provider. The final concern is based on application performance and effectively estimating the amount of resources that are required to be leased. This is similar to the capacity planning scenario, with the exception of the cloud capacity not being typically limited by the resources made available to the enterprise. Capacity planning is required to manage enterprise costs while also ensuring that the workload demands are met. This thesis focusses on devising techniques to handle application performance while efficiently utilizing cloud resources.

\subsubsection{Cloud Computing}

The National Institute of Standards and Technology (NIST) defines cloud computing as a model for enabling ubiquitous, convenient, and on-demand network access 
to a shared pool of configurable computing resources [6], providing highly available, reliable, and elastic services to cloud users. Elasticity is defined as the degree to which an application or system deployed inside a cloud infrastructure, autonomously adapts its capacity to workload demands over time [7]. To utilize the benefits of elasticity with ondemand cloud resources, a technique known as auto-scaling is used, where the number of resources utilized in supporting job execution in a cloud environment, changes automatically based on system load. While cloud providers allow resources to be provisioned based on demand changes, deciding on the appropriate number of resources is not a trivial task [8]. Auto-scaling with compute resources forms the focus of this research, with Chapter 3, Chapter 4, Chapter 5 and Chapter 6 describing auto-scaling techniques using only compute resources. Auto-scaling network resources in conjunction with compute resources is discussed in Chapter 7.

\subsubsection{Service Level Agreements and QoS Guarantees}

The on-demand nature of a cloud environment leads to significant cost savings for the organization as compared to deploying applications on-premise. However, the organization is also interested in ensuring that their requests, specific to the applications deployed on the infrastructure, are completed in a timely manner. One way to accomplish this is to introduce Service Level Agreements (SLAs) for the workload by allowing requests to specify an earliest start time and deadline for the requests. These types of requests are also referred to as advance reservation (AR) requests in the literature [9]. ARs are an important feature of clouds and distributed systems [10]. The requests used in this research have SLAs which include adhering to some request characteristics and have been described in more detail in Section 3.1.2. However, some requests may not have any 
deadlines associated with them. These requests are known as on-demand (OD) requests.

Although cloud computing has simplified capacity provisioning, it poses several challenges in the area of Quality of Service (QoS) management. QoS denotes the levels of performance, reliability, and availability offered by an application and by the platform or infrastructure that hosts the application. The challenge for a cloud provider offering its infrastructure to its customers is to maintain a QoS for guaranteeing metrics such as a low response time, high throughput and high service availability. QoS is imperative for cloud users expecting cloud providers to deliver the advertised quality characteristics, and for cloud providers, to determine the tradeoffs between QoS levels and operational costs. The SLAs that are considered by the resource management algorithms presented in this thesis capture the requirements of specific requests. Since an SLA is related to an individual request, this work also introduces the Grade of Service (GoS) criterion, which considers the overall QoS for the entire user application, encompassing all user requests. Further details about the GoS criterion may be found in Section 3.1.

\subsubsection{Service Models}

NIST defines three service models for cloud computing, as illustrated in Figure 1.1 and described next.

\section{Cloud Infrastructure as a Service (IaaS)}

This is a service that allows provisioning compute, storage and networks resources where the user can deploy and run arbitrary software, which may include operating systems and applications. IaaS resources are usually controlled via an API that allows cloud resources to be provisioned, removed or monitored.

2. Cloud Platform as a Service (PaaS) 
This is a service that allows users to deploy software applications onto the cloud provider's infrastructure. The software applications are written with popular programming languages and tools supported by the cloud provider. PaaS providers present users with an environment in which the operating system, an interpreter or a compiler to run the program are already pre-configured. The PaaS providers also provision the underlying server hardware and network infrastructure, allowing users to focus on the application development of their product or service.

\section{Cloud Software as a Service (SaaS)}

This is a service that provides access to a provider's applications running on a cloud infrastructure. The applications are accessible from various client devices through a web browser (e.g., web-based email). SaaS moves the responsibility of managing software and its deployment from end-users to third-party services.

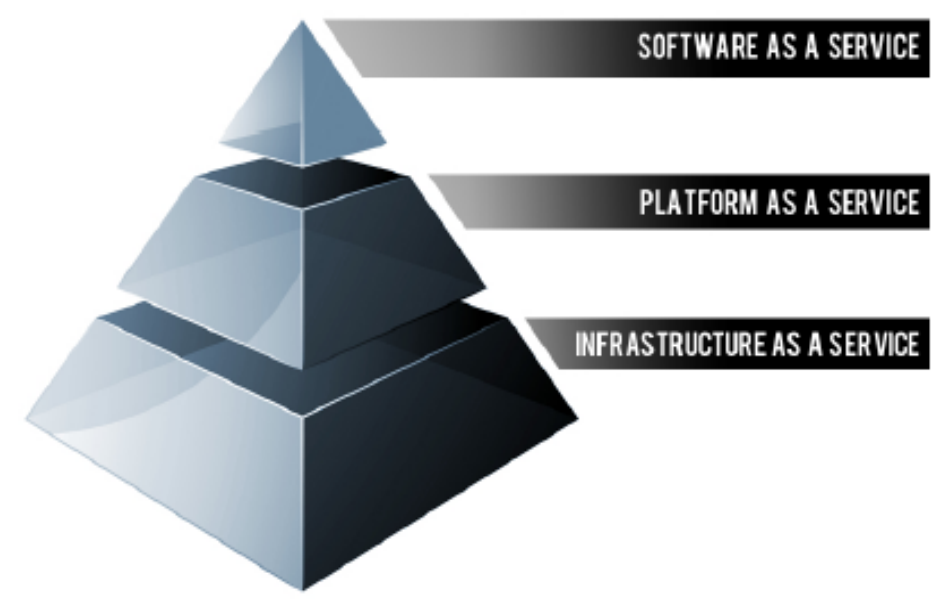

Figure 1.1: Service models for Cloud Computing [11]

\subsubsection{Pricing}

Cost is an integral component when optimizing the cloud environment. Pricing of cloud services allow users to choose a cloud service provider that best suits their 
requirement, by either reserving cloud resources in advance or by buying those resources on-demand. A cloud provider is equipped with a price-setting mechanism which sets the current price for the resource based on market conditions, user demand, and current level of utilization of the resource.

Pricing can either be fixed or dynamic depending on market conditions prevalent in the cloud environment [19]. For fixed pricing, instances are charged by the hour by many cloud providers including Amazon [2] and Microsoft [12]. In such cases, a fraction of an hour is counted as a whole hour. Therefore, part of the resource time remains unutilized if machines are released before a whole hour operation. In addition to the full hour principal, clouds now usually offer various instance types, such as high-CPU and high I/O instances [2]. Though fixed pricing is the dominant strategy today, dynamic pricing is used to handle unpredictable demand [13]. Regarding dynamic pricing, the authors of [14] present a revenue maximization framework to tackle the dynamic pricing problem in an IaaS cloud by modelling a stochastic dynamic program to calculate optimality conditions for setting the prices.

This research tackles the problem of handling this unutilized time due to idling resources by allowing users to rent resources by paying per second rather than per hour. This allows users to pay only for the time that the resource is utilized by their request. The broker earns a profit by allowing multiple requests to share the same resource, thereby minimizing the amount of idle for each resource. The charging for the resources and invoicing the end-user is the responsibility of the cloud vendor. With respect to cloud usage, the cost to the end-user is a combination of communication cost and the charges the user pays to the cloud vendor. Other pricing models in which users can pay only for a part 
of an hour of resource usage have also been recently introduced by some cloud service providers and are considered in Chapter 7.

\subsubsection{On-Demand IaaS Instances}

On-Demand instances from an IaaS allows a customer to pay for compute capacity by the hour with no long-term commitments. This enables the customer to operate without the overhead from the costs and complexities of planning, purchasing, and maintaining hardware and transforms what are commonly large fixed costs into much smaller variable costs.

\subsubsection{Reserved IaaS Instances}

Reserved Instances by an IaaS gives the customer an option to make a low, onetime payment for each instance the customer wants to reserve, typically for a period of 1year or 3-years. The customer, in turn, receives a significant discount on the hourly usage charge for that instance. After the one-time payment for an instance, that instance is reserved for the customer, and the customer has no further obligation after the upfront payment. Though reserved instances allow a cloud customer the option to make a one-time upfront payment for each instance, they are not suitable for users that want to lease resources for a short period of time.

\subsubsection{Network Pricing}

There are different pricing mechanisms utilized by different cloud providers when pricing network resources. A single data center network supports two types of traffic: (a) traffic flowing between external end systems and internal servers (known as North-South traffic [15]), and (b) traffic flowing between internal servers (known as East-West traffic [15]). Most cloud providers charge for network traffic entering and leaving the data center. 
Additionally, cloud providers are incentivized to reduce East-West traffic as it may lead to network bottlenecks. However, most public cloud providers do not allow end-users to reserve network bandwidth. Some software platforms for cloud management such as OpenStack [16], provide the capability to end-users to reserve bandwidth. Such capabilities for reserving bandwidth require wider adoption by major public cloud providers. Moreover, network resources used by public cloud providers are shared between many users; hence there are no guarantees for latency, bandwidth and failure handling.

\subsection{Motivation and Objectives}

The objective of this thesis is to introduce auto-scaling techniques that manage resources for the organization that have a variable workload demand. This thesis focusses on a homogeneous environment in which all compute resources are identical. Investigating a system with multiple types of computing resources forms an important direction for future research. The auto-scaling systems are deployed inside an entity referred to as the broker. The techniques focus on generating a profit for the broker while reducing the costs for the organization using the broker to auto-scale the resources acquired for the organization. In this thesis, the organization has been referred to as a Single Client Enterprise, that utilizes the services of the broker by sending user requests that need to be serviced by acquiring cloud resources, in accordance with the request SLA that includes a deadline. The motivation behind adopting an auto-scaling strategy is having the resources available when demand increases in addition to minimizing idling resources when demand decreases. Traditional approaches to handling peak demand involve over-provisioning or allocating a large number of resources. During off-peak hours, these resources would be idling, although the organization will still incur the cost of resource renting. Employing auto-scaling strategies address this challenge, reducing the costs incurred. Instead of forcing an organization to 
acquire the resources before deploying an application, resources may be incrementally added or removed as the demand for the application fluctuates over time. Moreover, instead of over-provisioning the application's infrastructure to meet the peak workload demands, the organization may employ auto-scaling techniques to control expenditure during low usage periods while still being able to satisfy peak demands during high usage periods. As shown in Figure 1.2, the organization may choose to over-provision (see Figure 1.2 (a)). This guarantees resource demands to always be met, in addition to the organization paying for the excess capacity which remains unutilized during low demand periods. The organization may choose to embrace the on-demand nature of the cloud by acquiring and releasing resources using sub-optimal techniques that result in paying more when capacity is excess and being unable to handle peak demand when capacity is lower than demand as shown in Figure 1.2 (b). The object of the thesis is to come up with a technique that only provisions as many resources as required and removes resources when no longer required, thereby meeting the organizations demands while minimizing spending (see Figure 1.2 (c)).
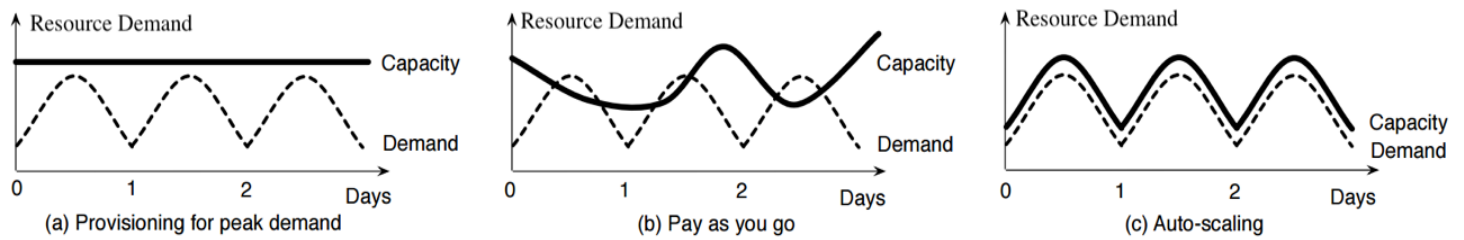

Figure 1.2: Benefits of Auto-scaling (Based on [17])

There are multiple objectives of this research. One of the objectives is to devise techniques for auto-scaling cloud resources using different approaches that are outlined next. 
- Reactive

- This technique auto-scales resources as resource demand fluctuates. The decision to auto-scale is based on certain criteria and the broker is made to 'react' to the request arrival. In this case, auto-scaling occurs after a specific change in system state has occurred.

- Proactive

- This technique auto-scales resources prior to when a change in resource demand occurs on the system. The decision to auto-scale is computed based on a time-series analysis made periodically using the history of request characteristics to forecast future request arrivals and their resource demands. In this case, auto-scaling occurs before the change in system state has occurred.

- Hybrid

- This technique uses a combination of the proactive and the reactive techniques to perform auto-scaling operations. When a new request arrives at the system and the resources controlled by the system are not adequate to service the request, new resources may be acquired reactively. Resources are also acquired proactively, based on the future demand computed by the system.

The three auto-scaling techniques are then used to accomplish a key objective of increasing the broker profit while reducing the user cost for the organization. The hybrid auto-scaling technique is developed in order to achieve the benefits of both the proactive and the reactive approaches in one system and is expected to improve the overall system 
performance in comparison to the pure reactive and pure proactive systems.

The second objective is to ensure that a certain Service Level Agreement (SLA) for the requests sent by the organization are met. The SLA is a contract set up between the organization and the auto-scaling entity, in this case, the broker. This is similar to a legal contract, that once accepted by all parties, must be met by the broker, even if it leads to the broker incurring a loss.

Chapter 3, 4, 5 and 6 in this thesis focus on auto-scaling only compute resources. However, an additional objective of the thesis is to auto-scale network resources in conjunction with compute resources. This is to address user requests which may require some data to be processed when executing the request. This data typically resides on a separate storage resource and needs to be transferred to the compute resource where the job is executing using the network. Controlling the data center network resource is not allowed when using typical public cloud providers like AWS or Azure. However, other cloud providers using OpenStack, for example, may allow their users to set bandwidth reservation as specified in their request [18]. Auto-scaling network resources is considered in Chapter 7.

The motivation for auto-scaling network resources is similar to auto-scaling compute resources. Hence, the broker earns a profit when selling the network resources to the organization. However, this work introduces a new goal of attempting to reduce the East-West traffic flowing internally inside the Data Center. Thus, the techniques investigated in this research allow compute resources to be placed near storage resources in order to minimize network activity, thereby reducing costs for SCE as well as costs for the public cloud provider. 


\subsection{Contributions}

To investigate the behaviour of the system for the proposed algorithms introduced in Section 1.2, an event-driven simulation was developed. Additionally, proof-of-concept prototypes were also developed for selected algorithms using resources from the Amazon public cloud. This research includes a detailed performance analysis of the proposed algorithms for various combinations of system and workload parameters using the simulator and the prototype. The contributions of this research to the state of the art are summarized next.

- Three novel auto-scaling algorithms that handle SLA driven AR requests as well as OD requests for compute resources.

- A reactive auto-scaling algorithm that scales the number of resources for the user in response to a change in the system workload.

- A proactive auto-scaling algorithm that uses past workload for the system to predict the characteristics of the requests that are expected in the future, subsequently using this forecast to decide on the number of resources to be deployed when these predicted requests arrive.

- A hybrid auto-scaling algorithm that combines the advantages of both the reactive and proactive techniques.

- A Grade of Service Criterion: Auto-scaling operations always consider the Grade of Service for the user's workload and maintain a strict criterion of accepting a given proportion of user requests that are guaranteed to be executed.

- Insights into system behavior and performance are obtained from experiments performed on proof-of-concept prototypes as well as simulations. Performance 
evaluation demonstrates that for the set of system and workload parameters experimented with, a profit is accrued by the broker. Furthermore, the broker reduces the user cost for SCE in comparison to a system in which users directly acquire their resources from the public cloud provider. A number of key insights resulting from the performance evaluation of the auto-scaling techniques is presented.

- Results demonstrate that the reactive techniques perform better in some cases while the proactive techniques perform better in other cases.

- Using a hybrid technique results in a higher broker profit in comparison to using only a proactive or a reactive technique.

- The thesis has focused primarily on compute resources. The research also demonstrates the ability of extending these auto-scaling techniques to handle network resources: it introduces a reactive auto-scaling technique that manages both compute and network resources while still enforcing the SLA and QoS requirements.

- A performance analysis demonstrates that a profit is accrued by the broker while decreasing the user cost.

- The auto-scaling technique helps reduce the East-West traffic in the Data Center that, in turn, reduces the utilization of the Data Center Network.

The results of this thesis are useful to tier 2 service providers and researchers.

The following publications have resulted so far from the research:

1. A. Biswas, S. Majumdar, B. Nandy and A. El-Haraki, "Automatic Resource Provisioning: A Machine Learning based Proactive approach," in Proc. of International Conference on Cloud Computing Technology and Science (CloudCom), Singapore, pp. 168-173, December 2014. 
2. A. Biswas, S. Majumdar, B. Nandy and A. El-Haraki, "An Auto-Scaling Framework for Controlling Enterprise Resources on Clouds," in Proc. of 15th IEEE/ACM International Symposium on Cluster, Cloud and Grid Computing (CCGrid), C4BIE Workshop, Shenzhen, China, pp. 971 - 980, May 2015.

3. A. Biswas, S. Majumdar, B. Nandy and A. El-Haraki, "Predictive Auto-scaling Techniques for Clouds Subjected to Requests with Service Level Agreements," in Proc. of IEEE World Congress on Services (SERVICES), New York City, NY, U.S.A, pp. 311 - 318, June 2015.

4. A. Biswas, S. Majumdar, B. Nandy and A. El-Haraki, "A Hybrid Auto-scaling Technique for Clouds Processing Applications with Service Level " in Journal of Cloud Computing, Volume 6, Issue 1, December 2017.

\subsection{Thesis Outline}

The layout of the thesis is presented as follows. Chapter 2 presents a literature survey of the related work that deals with auto-scaling and resource management techniques in the cloud environment. Chapter 3 discusses the architecture for the major components of the auto-scaling system.

Chapter 4, Chapter 5 and Chapter 6 present the techniques for reactive, proactive and hybrid auto-scaling respectively. These chapters introduce the respective broker architecture as well as outlining the algorithms for each of the techniques. These chapters also discuss the system and workload parameters used when performing experiments using the auto-scaling techniques. The performance of the respective technique and discussion of the performance results are also presented. Chapter 7 introduces the concept of autoscaling with network resources in addition to compute components. Chapter 8 concludes 
the thesis and outlines the directions for future research. 


\section{Chapter 2 Related Work}

The problem of dynamic provisioning of resources in computing clusters has been studied extensively. Cloud elasticity and auto-scaling may be viewed as a generalization of the same problem, extended to a cloud environment. Section 2.1 discusses a representative set of existing researches on resource managers in clouds, which are used to perform auto-scaling operations. Research related to the various auto-scaling techniques for a reactive, proactive and a hybrid auto-scaler for managing compute resources are described in Section 2.2, Section 2.3 and Section 2.4 respectively. Finally, Section 2.5 discusses the research related to auto-scaling the network component in a cloud/datacentric environment.

\subsection{Resource Managers}

An important aspect to consider when designing resource managers for the cloud environment is to utilize available resources efficiently.

Xu et. al. [19] describes a resource management framework called Anchor where resource management policies are separated from the management mechanism. The authors propose an algorithm for matching demands with VMs having different CPU, memory and storage resources based on user policies. The key contribution of this work is decoupling the user policies from resource management. OpenNebula [20], another resource management system for virtualized infrastructures, also decouples management policies with mechanisms for provisioning VMs. Auto-scaling decisions are made based on the conditions set by the service and not by individual requests. Haizea, proposed by Sotomayor et. al. [21] is a resource lease manager, providing leasing capabilities, such as handling ARs by allowing resource preemption. To satisfy SLAs, requests for resources 
have to be prioritized, queued, pre-reserved, deployed on external clouds, or even rejected.

Cost of VMs is an important consideration while acquiring and releasing resources. A framework called Aneka is proposed by Buyya et. al [10]. It provides users with an API for managing resources and expressing the business logic of applications by using programming abstractions. Aneka acquires, and releases resources based on the completion times of requests. If the current set of resources are unable to complete the newly arriving request before its deadline, additional resources may be acquired. Resources are released when certain threshold conditions are met. The provisioning algorithm of Aneka performs cost-optimization: allocating the minimum number of resources that enable the deadline to be met. Another research considers the cost of VMs is presented by Wang et. al. [22], who propose a system which employs a cloud brokerage service to reserve resources from a public cloud provider instead of acquiring them on demand. Their broker reserves resources at a bulk price that is lower than the on-demand price. The broker saves on costs by allowing multiple requests to be executed by one instance as well as maintaining a price gap between on-demand and reserved instance. Thus, the broker transfers these benefits to cloud users with heavy discounts while gaining profits from the achieved cost savings. To exploit the price benefits of reserved instances, the authors propose a set of dynamic strategies to decide when and how many instances to reserve, while maintaining a guaranteed performance for the user's application.

Each AR request in this thesis research has a deadline which needs to be met by the resource manager. When scheduled, requests are prioritized based on their deadlines to ensure SLAs are met. The research matches a request to a VM based on profit for the resource manager, referred to as a broker. The authors of [19], [20], and [22] are concerned 
with the management of resources in a cloud environment but do not use deadline based resource allocation. As in the research presented in this thesis, the work in [21] and [10] consider requests associated with deadlines as the same types of request as used in this thesis, namely AR and OD requests. However, none of these works [10], [19], [20], [21] and [22] use broker profit in making auto-scaling decisions.

This thesis also maintains a Grade of Service (GoS) in addition to auto-scaling resources, by applying the auto-scaling operation to the whole service by maintaining an overall proportion of requests that must be accepted. [21] also decouples the auto-scaling policy from the auto-scaler and considers QoS as a criterion that impacts request rejections A similar criterion is also used by [20], which acquires resources by measures the quality of service of an application. The other papers do not use a criterion similar to GoS when auto-scaling resources.

One of the goals of the thesis is to reduce user cost. This thesis proposes cost benefits using on-demand resources. Only [22] considers user cost as a metric when auto-scaling and attempts to reduce the user cost but does not consider profit for the resource manager that is considered in addition to user cost in the techniques described in this work. This research discusses dealing with network resources in addition to compute resources, when auto-scaling, whereas all other research papers described earlier only focus on scaling compute resources. Finally, this research uses machine learning in some techniques to predict future workloads in order to auto-scale resources. None of the other research papers discussed in this subsection consider proactive resource scaling.

Automatic resource provisioning may be classified into reactive rule-based mechanisms, proactive mechanisms and hybrid techniques. None of the other research 
papers discussed in this subsection have used machine-learning for performing autoscaling.

\subsection{Reactive Auto-Scalers}

Traditional techniques for reactive auto-scaling consist of creating rules for changing the number of resources. This requires a significant effort from the application manager by selecting a suitable metric to base the scaling decisions on. In some cases, additional metrics may be combined to arrive at a scaling decision. For example, an application may be utilizing the CPU and memory of a compute resource. If the workload demand for the application increases, it may utilize more CPU and memory from one or more compute resources. Scale-up rules in such a case would depend on the application manager setting a rule to scale-up, once a threshold for a metric has been crossed. However, selecting the corresponding thresholds requires a deep understanding of workload trends for each application required to be auto-scaled. Reactive auto-scaling mechanisms generally rely on such thresholds for automatically scaling of resources. Amazon's CloudWatch [23] utilizes such target metrics that are specified by its users to trigger action on reaching their thresholds. CloudWatch helps track metrics set by its users, collect and monitor log files and set alarms. CloudWatch also allows a system wide visibility into resource utilization, application performance and operational health which enables users to spot trends and make automated decisions based on the user's cloud environment.

Threshold based auto-scaling is also described by Dutreilh et. al. [24]. The authors suggest that thresholds need to be carefully tuned in order to avoid oscillations in the number of system resources (e.g. in the number of VMs or in the amount of storage assigned, a phenomenon known as thrashing). Oscillations may occur if the auto-scaling 
system keeps changing the number of resources in order to satisfy the threshold rules. To prevent this problem, [24] also introduces a cool-down or calm period, a time during which no further scaling decisions can be committed, once a scaling action has been carried out. Conditions in the rules are usually based on a single, or at most two metrics such as the average $\mathrm{CPU}$ load of the VMs, the response time, or the input request rate. The average response time of the application is the metric used; for example, in [24].

An auto-scaling algorithm [25] introduced by the company RightScale, proposes combining regular reactive rules with a voting process. If the majority of the VMs agree on that they should scale up or down, that action is taken; otherwise, no action is planned. After each scaling action, RightScale recommends a 15 minute-period of calm time because new machines generally take between 5 to 10 minutes to be operational. Chieu et al. [26] initially established reactive rules based on the number of active sessions, but this work was extended by following the RightScale approach: if all VMs have active sessions above the given upper threshold, a new VM is provisioned. If the number of VMs with active sessions is below a given lower threshold and with at least one VM that has no active session, the idle one will be shut down. As RightScale's voting system is based on rules, the technique is highly dependent on user-defined threshold values, and also, on the characteristics of the input workload.

Lim et. al. [27] advocates to narrow the range between thresholds when more frequent decisions are needed. instead of using static thresholding for their control policy. This framework has a target range for meeting the goal by defining a high and low threshold. The system makes scaling decisions when measured thresholds fall inside the target range. The authors also suggest that an external controller should be empowered to operate their 
own dynamic controllers, outside of the cloud platform itself, or perhaps as extensions to the cloud platform. This causes a decoupling of operations of the resource manager from the cloud provider's mechanism to prevent cloud platforms from becoming weak as user workloads vary.

Kouki et. al. [28] presents a system known as SCAling, which uses an analytic model that looks at a global objective when auto-scaling. SCAling is an approach for auto-scaling driven by SLAs. The global objective is to manage cloud elasticity, i.e., ensure that the number of used instances increases seamlessly during demand spikes to maintain performance, and decreases automatically during low demand periods to minimize costs. They create a control loop that follows a MAPE-K (Monitor, Analyze, Plan, Execute and Knowledge) control loop model. The SCAling Monitor function gathers information about infrastructure (e.g., number of instances at each tier, instance lifecycle), workload and SLA parameters. The gathered information is raised periodically to the SCAling function which analyzes whether it is necessary to re-plan capacity. It handles the trade-off between profit for the broker and customer satisfaction levels without requiring manual intervention.

Previous work by the author of this thesis and other researchers presented by Melendez et. al. [29] has described reactive auto-scaling of resources based only on blocking ratio (B). More details about B are discussed in Section 4.1. A scale up operation is performed when the value of B goes above a particular threshold set by the user and a scale down operation is performed when B goes below a certain threshold. As with [27], a high and low threshold is used to avoid thrashing.

Wang et. al. [22] proposes a system which employs a cloud brokerage service to reserve resources from a public cloud provider instead of acquiring them on demand. Their broker 
reserves resources at a bulk price that is lower than the on-demand price. The broker shares the resources with multiple users while offering them per second billing. The goal of the thesis is to present different techniques to reduce user cost. Novak et. al [30] also present a technique for reactively auto-scaling compute resources that reduce costs for the user. They present two different approaches for reducing user cost, one that is cloud provider dependent and the other being cloud provider independent. Costs are reduced by decreasing the time to launch a VM even as user demand increases, thereby allowing users access to resources when they desire.

The thesis introduces a grade of service that ensures that the blocking ratio for the system remains below a desired level specified by the users. Thus, the resource manager maintains the specified grade of service set by the single client enterprise (SCE), thereby providing SCE with a service level GoS guarantee. However, [23], [22], [25], [27] and [30] do not provide a guarantee for overall service performance when auto-scaling. Although [29] provides a blocking ratio, and [28] uses a global objective similar to the grade of service, they do not consider broker profit as a metric used in auto-scaling which forms the basis for resource management described in this thesis.

The problem of oscillation is described earlier in this section with [24] solving the issue by introducing a cool-down period between successive auto-scaling operations. This thesis presents techniques that do not use a cool-down period by instead perform auto-scaling operations based on the expected profit for the broker. Hence, the broker controls the resources and acquires only when resources are deemed profitable. However, the resources are only released at the end of the "paid hour" (more details may be found in Section 4.1). Hence, resources are not acquired and released in succession. 
One of the goals of this research is to reduce the cost for the user. Reduction of cost is also a focus of [22], [29] and [30]. While [29] considers ARs, [22] and [30] do not consider ARs while provisioning resources.

\subsection{Proactive Auto-Scalers}

Proactive resource management systems attempt to predict future workload while making scaling decisions. Moore et. al. [31] uses a proactive technique for resource provisioning that implements a real-time performance monitoring framework known as Platform Insights. which is responsible for performing auto-scaling decisions for their cloud platform. The proactive elasticity controller is designed to extend the functionality offered by reactive auto-scaling rules by generating predictive models based on them. The proactive controller comprises of three models: a time series forecaster and two incrementally updateable Naive Bayes models. For the time-series predictions, the authors use a Support Vector Regression (SVR) regression algorithm.

Time-series analysis is the most used approach to build proactive auto-scaling techniques [32]. The accuracy in prediction (e.g. the upcoming requests) depend on the selected prediction technique and choosing the appropriate parameters. For example, the prediction technique needs to maintain an appropriate history window comprising of past events to make predictions. If the history window is too short, predictions will be inaccurate and if the history window is too long, the prediction operation will increase the time complexity of the algorithm. Additionally, the prediction technique needs to select a prediction interval which determines how often the technique is invoked. Time series analysis techniques are desirable for implementing auto-scalers, as they can effectively predict future demands arriving to elastic applications. Predictive techniques are desirable because they enable 
resource to be acquired in advance, thus avoiding additional waits due to the time required to start new resources.

This thesis uses a proactive auto-scaling technique for workload prediction using a time series analysis. Time-series analysis can be used to find repeating patterns in the workload or to forecast future values. In order to use time-series analysis in auto-scaling, a performance metric (such as the number of requests per time unit) is periodically sampled at fixed intervals. The result will be a time-series containing a sequence of last observations. Time-series algorithms extrapolate this sequence to predict future value. Some of the techniques used for this purpose in the literature are Moving Average, Autoregression (also referred to as ARMA), exponential smoothing, and machine learning approaches [8]. Bhagavathiperumal et. al [33] also use an approach based on ARMA to devise a proactive workload predictor that attempts to provisions resources prior to the increase in demand during peak hours.

Nikhravesh et. al. [34] uses a proactive auto-scaling technique with time series analysis which proves a hypothesis on prediction accuracy of predictive auto-scaling systems for the IaaS layer of cloud computing. According to the hypothesis, prediction accuracy of predictive auto-scaling systems can be increased by choosing an appropriate time-series prediction algorithm based on incoming workload pattern. The authors utilize two timeseries prediction techniques known as Support Vector Regression (SVR) and Neural Networks (NN). The results of their experiments show that SVR has a better prediction accuracy in the environments with periodic and growing workload patterns, while $\mathrm{NN}$ outperforms SVR in forecasting unknown workload patterns. The authors also propose an architecture for a self-adaptive prediction framework using an autonomic approach which 
can choose the best prediction technique based on the workload pattern, which leads to more accurate prediction. The authors emphasize more on the prediction techniques used when predicting the time-series for the workload.

Some of the proactive auto-scaling techniques that use time-series analysis deal with pattern identification for detecting patterns in the workload. Gong et. al. [35] introduces an auto-scaling system known as PRESS, which tracks resource requirements of a cloud application and predicts resource demands in the near future using two complementary techniques. The first technique employs a signal processing technique to identify repeating patterns called signatures that are used for its predictions. If no signature is discovered, PRESS employs a second technique to detect short-term patterns in resource demand using a discrete-time Markov chain to predict demand for the near future. The resource prediction models are updated when resource consumption patterns change. Additionally, the authors of [35] also use a broker model with the broker charging the user and the broker paying a charge to the cloud provider. The profit-rate of the broker is calculated by starting with the revenue obtained from renting out the resources to the user and subtracting any penalties for SLA violations.

Regression models are able to predict characteristics of a time-series such as the input workload as described in the other prediction approaches. In this thesis, a regression model is used to predict the future workload. However, other variables in a cloud data center, such as CPU usage may also be used for prediction. Such an alternative approach has been adopted by Fang et al. [36], where the authors predict future CPU usage of resources. Their model has high prediction accuracy and uses a fine-grained/coarse-grained approach to solve the resource provisioning problem in cloud data centers. A fine-grained prediction 
approach, while being more accurate is computationally more intensive than a coursegrained approach. The resource usage of the CPU for a duration of time is extracted as a time series data and inputted into a load predictor model. To predict the short-term resource demands with the CPU utilization, the compute resource's usage is represented as a time series, each value in the series being between 0 and 100 percent, representing the CPU usage at a time instant. However, the prediction model results in a large amount of computations being performed, forcing the algorithm to have a much higher time complexity that other prediction techniques. The authors utilize a coarse-grained resource provisioning approach to reduce the time complexity of their algorithm when confronted by a sudden spike in their workload.

The particular contribution of this thesis is to devise a proactive auto-scaling technique that learns on-line while performing the prediction operations. However, the scaling decisions are based primarily on profit for the auto-scaling controller (broker) whereas the other works discussed in this sub-section do not incorporate cost-awareness in their autoscaling decisions. Moreover, this research handles AR requests that allow users to specify an SLA individually for each request. Jain [35] only includes scaling metrics of workload arrival rate and QoS while auto-scaling resources and do not consider resource costs. This thesis focusses more on the decision-making aspect of auto-scaling that deals with managing resources and deciding on when to auto-scale. This research uses Linear Regression (LR) as the main time-series prediction technique while also comparing its performance with that of SVR.

As in this research, the work presented by Guo et. al. [38] also use a broker based model and allows a request to miss deadlines employing a penalty for the service provider for 
violating SLA. The techniques described in this thesis also uses a broker, but all requests that are accepted are guaranteed to be executed. These techniques are free from SLA violations and guarantee the execution of all accepted requests by their respective request deadlines. The resource manager described in this thesis also considers both compute and network resources whereas all the other research papers discussed in this sub-section only focus on scaling only one type of resource, compute or network.

\subsection{Hybrid Auto-Scalers}

Proactive auto-scaling techniques described in Section 2.3 can be combined with reactive techniques like those described in Section 2.2. For example, Moore et. al. [37] propose a real-time cloud capacity framework, offering a hybrid elasticity controller employing both reactive rule-based and proactive model-based elasticity mechanisms in a coordinated manner. The approach is validated by using traces based on two real datasets. In both cases, the system provisions resource for the application server tier more appropriately than the reactive controller alone, yielding very few QoS violations and consistently maintaining a high CPU utilization. The hybrid controller examines the scale up condition which in a pure reactive auto-scaling environment would be used to acquire new resources and builds incrementally updateable predictive models to enable the system to proactively scale up before the scale up condition is met. The predictive model is also used to calculate the minimum number of resources required during the next 30-minute time interval in order to have a probability of less than $5 \%$ chance of reaching the scale up condition determined by the reactive component of the controller. This methodology allows the system to scale in as well as out. Whenever the predictive model fails, autoscaling decisions are left to the reactive controller. The research presented in [37] 
demonstrates that, compared to a pure reactive controller, or a pure proactive controller, the hybrid controller is able to make better provisioning decisions.

Urgaonkar et. al. [38] proposes an alternate hybrid control mechanism that incorporates both a proactive controller and a reactive controller. The proactive controller maintains the history of the session arrival rate seen. Provisioning is done prior to each hour based on the worst load seen in the past. Their workload predictor is based on a technique that uses past observations of the workload to predict peak demand that will be seen over a period of $T$ time units. With $T=1$, the predictor estimates the peak demand that will be seen over the next one hour, at the beginning of each hour. A histogram is generated with the past workload for each hour using observations for that hour from the past several days. Each histogram yields a probability distribution of the arrival rate for that hour. The peak workload for an hour is used for estimating the arrival rate distribution for that hour. The proactive provisioning technique allocates capacity to handle the worst-case load using the tail of the arrival rate distribution to predict peak demand. The reactive controller acts on short time scales to increase the resources allocated to a service in case the predicted value is less than the actual load that arrived. The reactive technique is invoked once every few minutes and may even be invoked on-demand by the application if the proportion of requests services drops beyond a threshold.

Iqbal et al. [39] proposes a hybrid scaling technique that utilizes reactive rules for scaling up (based on CPU usage) and a regression-based approach for scaling down. The authors assume a multi-tired web application with a web server and a database server that needs to be auto-scaled. After a fixed number of intervals in which the response time requirement is satisfied, they calculate the required number of application-tier and 
database-tier instances using a polynomial regression technique. The authors propose a reactive system for scale-up operations and a proactive system for scale-down operations. This is different from all other approaches that have been discussed thus far. The system uses a heuristic approach coupled with active profiling of the CPUs of virtual machinehosted application tiers for identification of bottlenecks. Resources are auto-scaled reactively based on thresholds placed on CPU utilization. If the system satisfies the response time requirements for $k$ consecutive intervals, it uses the predictive model to identify any over-provisioned resources and if appropriate, scales down the overprovisioned tier(s).

This thesis presents a hybrid approach that also improves performance as compared to a pure proactive or pure reactive approach. However, the scaling decisions are based primarily on profit for the auto-scaling controller whereas the other researchers do not incorporate cost-awareness in their scaling decisions. Moreover, this thesis handles AR requests that allow users to specify a SLA individually for each request, which are not considered in [38] and [39]. Although, [37] handles advanced reservations it does not handle requests with completion time deadlines that are an important component of the SLA considered in this research. In [38], the authors monitoring the demand for each hour of the day to capture time-of-day effect of a variable workload pattern and observe two distinct workload demands. A similar workload is used in this thesis to simulate the diurnal rate of request arrival. However, this thesis also auto-scales by using a differential pricing mechanism for end users to achieve a profit for the intermediary enterprise (using a broker).

\subsection{Network Auto-scaling}

Network auto-scaling is generally not supported on today's public cloud. Network auto- 
scaling and associated supporting technology have recently started receiving attention form researchers. A representative set of works is presented. Papers [40] and [41] focus on network auto-scaling and [42], [43] focus on auto-scaling of both compute and network resources. Bandwidth reservation, an important technique required for network autoscaling is the subject of attention in Section 7.1.1.

Auto-scaling of compute resources can be effectively performed using the research techniques described in Sections 2.1, 2.2, 2.3 and 2.4. However, auto-scaling the network resources inside a data center with bandwidth guarantees on shared network infrastructure is different from the auto-scaling of compute resources. This is because a request to use a compute resource requires that instances may be leased to one tenant for a fixed period of time. However, in the case of the network resources, the same request may have different network bandwidth reservation requirements on different links, depending on the placement of the compute resource relative to other resources, which are communicating with the compute resource over the network links.

An early network autoscaling framework, known as B4, is devised by Jain [40]. This paper presents an approach to separate the network's control plane from the data plane to enable rapid deployment of new network control services. A control plane carries information to the routers, whereas a data plane transfers network traffic. To establish a relative priority, B4 creates a contract between an application and itself. This contract specifies the bandwidth allocation to an application given the network demand's priority, which the paper terms as "fair share." The network auto-scaling service in B4 then autoscales bandwidth among these competing applications based on priority and variable network demands. The bandwidth reservation model attempts to minimize the East-West 
traffic in the Data Center while also improving the network utilization for the Data Center.

One of the main challenges with network auto-scaling is achieving network isolation, which isolates the traffic generated by one user from other traffic. Dalton [44] creates a network management framework called Andromeda that separates the data and control planes, similar to [40]. For isolation, they run separate control stacks in every cluster. The paper defines a cluster as a collection of collocated machines with uniform network connectivity that share the same hardware failure domain. Andromeda introduces a software called "Hoverboard" which allows on-demand network requests to provision network links between VMs, and auto-scales network resource as and when required. Andromeda consumes a few percent of the CPU and memory on a physical host. The Andromeda control plane maintains information of the current location of every VM, which helps make efficient routing decisions, thereby minimizing East-West traffic.

Rahman et. al. [41] have looked at auto-scaling network resources to reduce leasing costs while maintaining the QoS of the application. They employ a machine-learning based approach to predict future workloads to reserve bandwidth ahead of time. Their machinelearning algorithm looks at historic auto-scaling decisions for network resources and has a high accuracy for the prediction of network demand. The authors also measure the QoS of the network in terms of a metric they define as "Degraded QoS," which is the cumulative time (in minutes) a network resource spends in deployment, not able to meet network demands, and results in QoS violation. Their goal is to reduce the network leasing cost, and they show improvement over prior work. However, they don't provide a bandwidth guarantee every time, especially when their prediction algorithm makes an error. To solve the bandwidth, guarantee issue, Guo et. al. [42] propose a framework called SecondNet, 
which focuses on providing bandwidth guarantees among multiple VMs in a multi-tenant virtualized data center. The authors propose a virtual data center (VDC) as the unit of resource allocation for multiple tenants in the cloud. SecondNet achieves high scalability by moving information about bandwidth reservations from switches to server hypervisors. Additionally, SecondNet allows resources (compute and network) to be dynamically added to or removed from VDCs. SecondNet supports scaling down network resources by releasing unneeded network resources. Scaling up network resources is not as trivial, and there are two cases: increasing network resources for existing VM-pairs, or adding new VMs since they work with the VDC as a whole. The paper specifies an SLA for computation and bandwidth requirements for the VMs, and respective SLAs are monitored by a VDC manager.

Hasan et. al. [43] describes a reactive auto-scaling system called integrated and autonomic cloud resource scale, or IACRS. The IACRS framework correlates metrics from three domains: network, compute and storage in making scaling decisions. IACRS supports a thresholding mechanism that integrates performance metrics from multiple domains in making scaling decisions and scales network resources in combination with compute resources. For example, CPU load is correlated with response time over network links. Auto-scaling of resources occurs when the threshold for rules set by the users of the cloud system are crossed. The paper concludes by stating that spurious auto-scaling may be prevented by grouping together multiple metrics belonging to resources that are related to each other.

This thesis allows a controller to provision the network bandwidth in a way that provides a guaranteed bandwidth between servers. The focus of this thesis is to present various auto- 
scaling techniques whereas other researchers in [41] and [42] present their frameworks for representing the network resources as a shareable resource when making network autoscaling decisions. The main limitation of [42] is that its performance may depend on the physical topology of a network. This thesis auto-scales both compute and network resources without any limitations of the physical connectivity of the network. Hence, network bandwidth is reserved only if the request is accepted by the broker based on broker profit or grade of service criterion. [41] monitors a QoS parameter (response time) to maintain the desired quality for an application. While this maintains the QoS for a single workload, the authors do not look at the performance of the service as a whole. In this research, the auto-scaling system maintains the desired service QoS specified by the user, even at the expense of losing revenue. Research presented in [40] and [44] auto-scales the network resources by moving the control requests through a control plane. However, the network reservations are made using an API provided by the cloud provider, which may reserve the network resources using a separate control plane. Both the papers, [40] and [44] also aim to reduce the East-West traffic, which is also a motivation for the research presented in this thesis. However, the two papers do not discuss cost implications for the user, as well for an entity like the broker, which aims to accrue a profit. Finally, [43] employs a reactive auto-scaling strategy similar to the one discussed in this thesis, since the SLA requirements for the request include at both the compute and network demands, when auto-scaling. However, [43] does not look at resource costs or attempt to reduce the East-West traffic flowing through the Data Center Network. 


\section{Chapter 3 The Auto-scaling System}

This chapter describes the auto-scaling system, along with its different components. As introduced in Chapter 1, the auto-scaling systems are deployed inside an entity referred to as the broker. This chapter discusses the mechanisms that concern the broker's interaction with the client enterprise and the public cloud provider. The objective of the chapter is to present the common features used when discussing the auto-scaling research techniques that are covered in the following chapters. Each of the chapters, Chapter 4, Chapter 5, Chapter 6 and Chapter 7 that follow this one present research techniques that are based on the material covered in this chapter. This chapter introduces concepts that are used when auto-scaling is performed with only compute resources. Some additional background is provided in Chapter 7 when discussing auto-scaling network resources with compute resources.

This chapter is divided into the following sub-sections. Section 3.1 provides an overall system overview, while Section 3.2 presents a description of different pricing mechanisms used in this thesis. Section 3.3 presents a description of the other systems which have been used for comparison with the auto-scaling systems in the subsequent chapters.

\subsection{System overview}

An overview of the system is presented in Figure 3.1. An Intermediary Enterprise (IE) is responsible for handling requests sent by users from an organization known as the Single Client Entity (SCE). IE uses the broker which controls resources acquired from the public cloud to form a "private" cloud which is also referred to as a Virtual Private Cloud (VPC) in the literature [45]. From here on in, this thesis will be referring to IE as the private 
cloud provider. Typically, the broker runs in one of the instances of the public cloud acquired by the intermediary enterprise or in an external location controlled by the intermediary enterprise. However, the broker may choose to run in an alternate location as well, outside the cloud provider. In such scenarios, the broker must possess the ability to communicate with the resources of the VPC. Users inside SCE send their requests to the broker over the Internet. The broker allows the users to execute their requests on instances acquired from the public cloud provider. The broker charges the users of the private cloud a higher price per unit time than the public cloud provider in order to earn a profit. However, the broker charges users per second rather than on an hourly basis as used in the charging scheme of the public cloud provider. This ensures that users only pay for time their request is executing. The goal for this broker is to maximize profit for the private cloud provider (IE) while attempting to reduce the cost for the client enterprise (SCE) that comprises the users for the private cloud. The reduction of cost is determined by comparing with a system where, instead of renting instances from the private cloud provider, the user rents resources directly from a public cloud provider. This is discussed in more detail in Section 3.3.

The described approach allows the broker to share resources between different user requests. The broker has two responsibilities, which include handling the matchmaking and scheduling of requests along with managing the auto-scaling of the resources. The operations of matchmaking and scheduling deal with finding a suitable resource for an incoming user request and adding this request to a schedule which determines the start time of the request (further described in Section 3.1.4). Utilizing the principles of auto-scaling as described in Section 1.2, the broker dynamically controls the number of resources using 
a threshold-based mechanism or utilizing a prediction system based on a machine learning approach used to predict future workloads. The two techniques of using a threshold, or predicting the workload are described in detail in Chapter 4 and Chapter 5, respectively. Chapter 6 covers the use-case of combining the threshold-based mechanism with the prediction mechanism to improve auto-scaling performance.

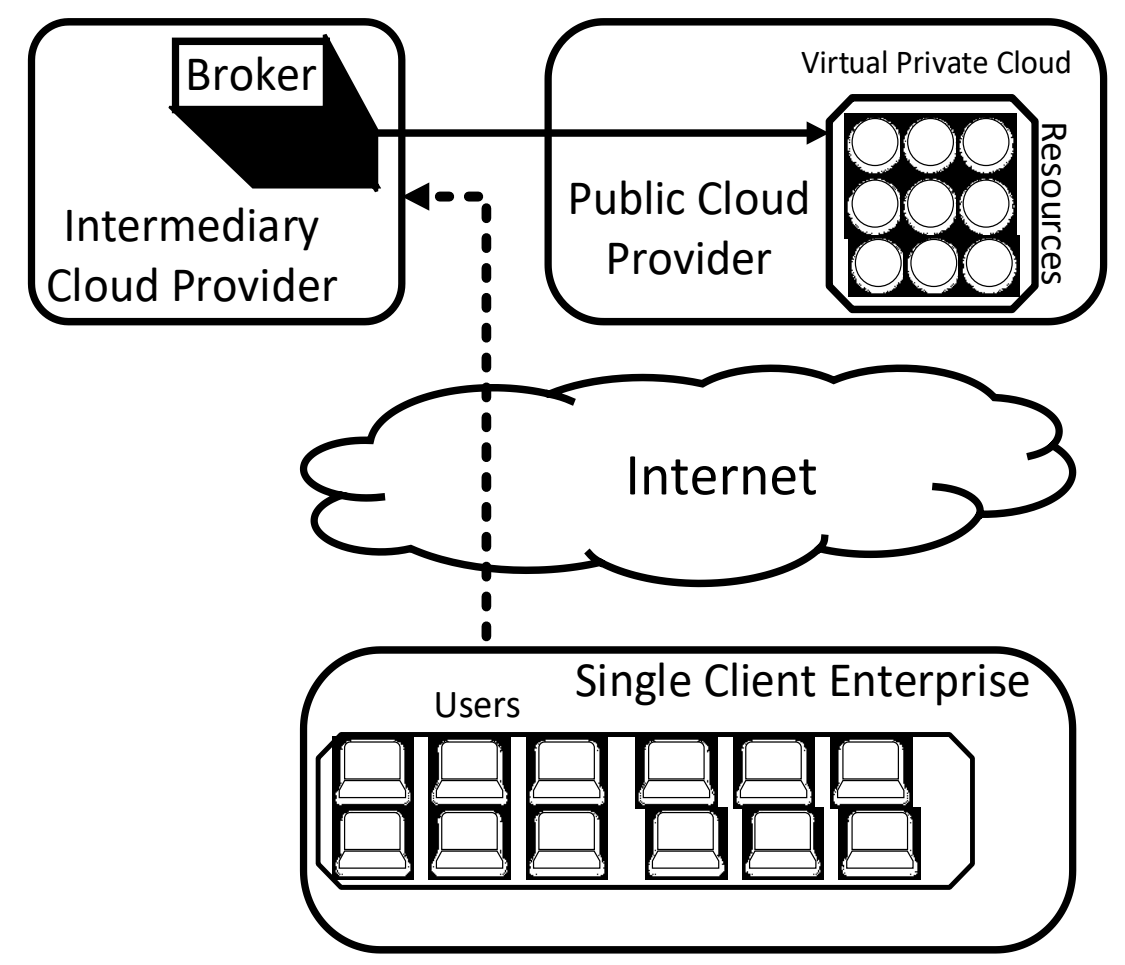

Figure 3.1: System Overview

The primary criterion used in this research is to auto-scale resources based on profit accrued by IE. In addition, another criterion for acquiring resources based on a Grade of Service (GoS) specified by the client enterprise is used. Adhering to the GoS criterion assures the client enterprise that a given proportion of their requests is guaranteed to be executed, even though such executions may result in a loss for the broker. Although the system described in this research uses the blocking ratio $(B)$ for describing the GoS, the framework can be adapted to other metrics as well. $B$ is the proportion of requests that 
cannot be completed before the expiry of their deadlines and are therefore rejected by the system. Maintaining the GoS implies keeping the blocking ratio below a certain threshold, thereby guaranteeing execution for a given proportion of the requests. Similar GoS criterion have been used by other researchers [46]. Auto-scaling using GoS was researched in a previous work [29] by the author of this thesis along with his supervisors dealing with reactive auto-scaling. In the paper, the system scaled up resources when the value of B went above a specified threshold and scaled down when the value of B went below the threshold.

\subsubsection{Broker Components}

The various components internal to the broker that aid in making auto-scaling decisions are described next. The broker controls $N$ resources at a given point in time: $\left\{\operatorname{Res}_{1}, \operatorname{Res}_{2}, \ldots \operatorname{Res}_{N}\right\}$, where $N$ changes dynamically with user demand for resources. This sub-section presents the common components used inside the broker. Although the different auto-scaling techniques proposed in this thesis differ in the implementation of the respective auto-scaling algorithms, all three of the proposed reactive, proactive and hybrid approaches use a number of common components that are described next.

- Decision Maker (DM) - This component is responsible for making the decision of when to perform the auto-scaling operation and is shown as one of the components of the broker in Figure 3.2. This component comprises of:

○ Auto-scaler - This sub-component implements the auto-scaling algorithm for the respective reactive, proactive or hybrid system.

- Dynamic Resource Pool Manager - This sub-component is 
responsible for the process of acquiring and communicating with cloud resources. Further details may be found in Section 3.1.5.

- GoS Module - This sub-component is responsible for maintaining the value of $B$ lower than the GoS set by SCE. This component also maintains the current value of $B$ achieved by the system and decides whether the request must be accepted even though it does not accrue a profit for the broker. Further details are found in Section 4.1.

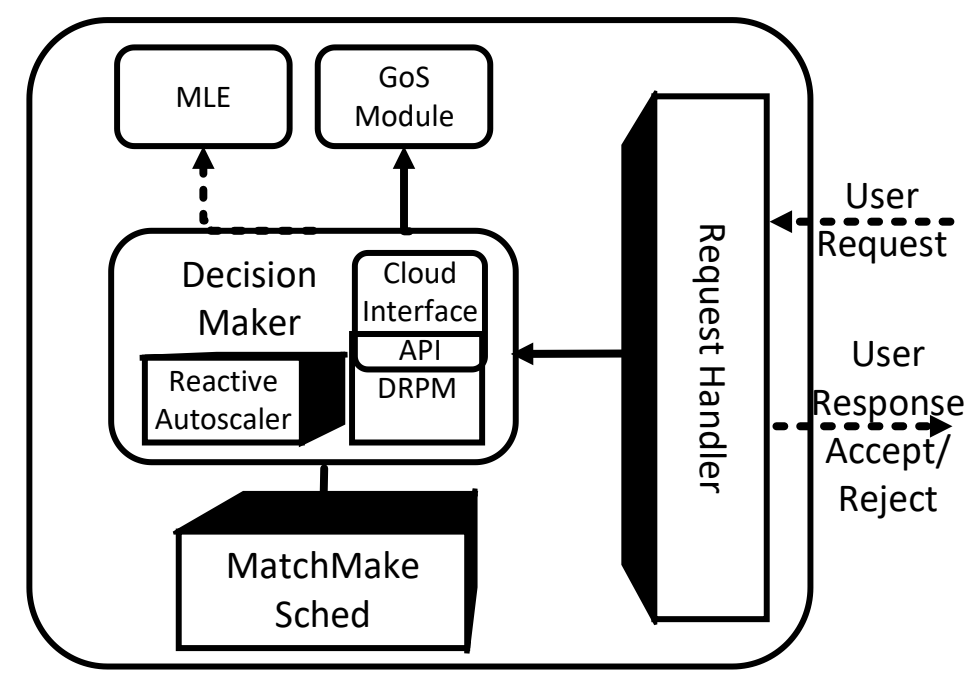

Figure 3.2: Broker Architecture

- Machine Learning Engine - This sub-component (used in the proactive and hybrid auto-scaling algorithms) is responsible for predicting the upcoming requests by using an analyzing the trends from past request arrival patterns. Further details are provided in Section 5.1.

- Request Handler $(\mathrm{RH})$ - This component handles incoming requests and forwards them to DM. The component also sends the response of DM back to the user that sent the request. A detailed discussion of this component is provided in Section 3.1.3. 
- Matchmaking and Scheduling (MMS) - This component is responsible for selecting a resource and scheduling the request on the resource. MMS is discussed further in Section 3.1.4.

\subsubsection{Request Characteristics}

The requests are sent by SCE to the broker, which subsequently decides to accept or reject the request based on certain criteria as determined by the matchmaking and scheduling algorithm. The characteristics of the requests include:

- $\quad$ Arrival Time $(A T)$ - The time at which the request arrives on the system.

- $\quad$ Earliest Start Time (EST) - The earliest time the request is allowed to begin execution.

- $\quad$ Service Time (ST) - The time taken by the request to execute.

- $\quad$ Deadline $(D L)$ - The time by which the request must complete its execution. DL is an important component for the SLA for the request.

- $\quad$ Type (T) - The type of the request: AR or OD.

Similar characteristics for requests have been used by other researchers as well [47]. When a request is scheduled, MMS computes two additional characteristics of the request so that it may utilize this information to facilitate scheduling requests:

- $\quad$ Scheduled Start Time (SST) - The time at which the request is scheduled to begin execution.

- $\quad$ Scheduled Finish Time (SFT) - The time at which the request is scheduled to complete execution.

In addition, as explained in Section 1.1.2, a request of OD type does not have an earliest start time or a deadline. OD requests may start at any time after they arrive on the 
system. OD requests can thus be associated with a start time that is equal to its EST. Also, to matchmake and schedule them by using a resource manager that handles ARs, OD requests are given a very large deadline that can never be reached. Matchmaking and scheduling are discussed further in Section 3.1.4

\subsubsection{Request Handler}

The request handler $(\mathrm{RH})$ is the first component that interacts with a user request. The broker is operated as a service on the public facing side in the public cloud environment. Users from a single client enterprise send their requests to IE via the broker over the Internet. Once the requests reach the broker, they are directed to RH. The primary responsibility of $\mathrm{RH}$ is to receive the requests from users and forward those requests to MMS which decides whether to accept or reject the request. OD request are always accepted because they do not have deadlines and can be executed at any as explained in Section 3.1.2. The decision of whether or not to accept an AR request is made by the matchmaking and scheduling algorithm that is discussed in the following section. The decision of whether the request will be accepted or rejected is communicated back to the user.

\subsubsection{Matchmaking and Scheduling}

Once a request enters MMS, a matchmaking algorithm determines a resource on which the request can be executed. A matchmaking algorithm allocates requests to be executed on a resource that is selected from a resource pool. A scheduling algorithm determines the order in which the requests allocated on a given resource are executed. The component uses a first fit (FF) strategy for matchmaking and an Earliest Deadline First (EDF) strategy for scheduling [48]. The architecture for MMS allows any other algorithm 
for matchmaking and scheduling to be used as an alternative. The first fit algorithm scans the resources acquired by the system sequentially and allocates the request to the first resource capable of meeting the request deadline. Each time a resource is considered by the matchmaker, the system runs the scheduling algorithm. This is due to each resource having a queue of requests that are already scheduled to be executed at a pre-determined time. When such a resource is selected, the new request is scheduled for execution along with the existing requests that were already queued for execution in the resource, ensuring that all AR requests in the queue are compliant with their earliest start times and deadlines.

With EDF, the requests allocated on a given resource are scheduled in accordance with their deadlines. A request with the shortest deadline is executed first followed by the one with the next shortest deadline and so on. If the newly arrived request can be allocated and scheduled on an existing resource such that its deadline can be met, the request is accepted. If the requested cannot be scheduled, then the auto-scaler is invoked to determine if a new resource should be acquired. OD requests are treated as a special case of AR requests in the system where the deadlines are flexible as described in Section 3.1.2. Since OD requests have flexible deadlines, they may be scheduled after all AR requests have completed execution. OD requests are scheduled similar to AR requests but may be rescheduled to allow AR requests to execute before OD requests are executed. The matchmaking and scheduling algorithms are not formulated by the author of this thesis but have been described in literature. Further details of these algorithms are discussed in the Appendix, but the interested reader is additionally referred to [47] that describes similar scheduling and matchmaking algorithms. Once requests have been scheduled, they must be completed. Thus, the broker guarantees execution of a request which has been accepted 
by the system, within the specified deadline.

Both the matchmaking and scheduling algorithms described in this section are based on user estimates of job execution times. Such estimates are often error prone [49]. Handling these inaccuracies is a responsibility of the matchmaking and scheduling algorithm used by the system and not auto-scaling algorithm that this thesis focuses on. Farooq et. al. [50] have described methods for handling such inaccuracies by using a prescheduling mechanism based on overbooking and a run time exception handler. Such mechanisms can be incorporated in the matchmaker and scheduler deployed by MMS that is not the subject of attention of this paper. Using the auto-scaling techniques in conjunction with such matchmaking techniques available in the literature, such as [50] forms an interesting direction for future research.

\subsubsection{Dynamic Resource Pool Manager}

The Dynamic Resource Pool Manager (DRPM) handles the task of communicating with the public cloud provider for acquiring or releasing resources using a cloud interface API as shown in Figure 3.2. DRPM possesses the ability to acquire resources from a cloud provider, as long as it is authorized to do so. This component alleviates the responsibility of the other broker components from interacting individually with the public cloud provider, and DRPM offers a single API to the broker to request for additional resources. Once a request is received, DRPM uses the API exposed by the cloud provider to acquire the resources and transfer the details of the newly acquired resource to the broker.

This research assumes that a request made by the broker at any time for an additional resource will be fulfilled by the public cloud provider. However, additional considerations are required for public cloud providers that bound the maximum number of 
on-demand resources that can be acquired at the same time. DRPM, in order to enforce an admission control mechanism, imposes a limit on the number of resources that may be controlled by the broker at any instant. In this research, the limit for the maximum number of resources acquired at the same time is set to 1000 resources. This limit is never reached with the workload used for running the experiments described in this thesis. In the unlikely event a higher workload demand forces the number of resources acquired by the broker to reach the limit imposed by DRPM, resources will no longer be available to the broker. Consequently, requests will be rejected by the broker, that may result in a violation of the GoS criterion set by SCE. Thus, the maximum number of resources that can be acquired by DRPM at any time will determine the upper bound on the workload intensity that can be supported by the broker.

\subsection{Broker Pricing Model}

The broker, which is hosted in the intermediary enterprise managing the private cloud, accesses resources from a public cloud provider. As discussed in Section 3.1, the broker acquires a number of resources from the public cloud provider, which forms into a resource pool making up the VPC and manages those resources. As discussed earlier, the goal of the system is to earn a profit for the broker (intermediary enterprise) while offering cost savings to the User. The system achieves this by using a differential pricing mechanism and an effective and efficient auto-scaling of resources. The public cloud provider charges the broker $c \_p u b$ dollars an hour per resource. However, the broker charges the user $c \_p v t$ dollars per second. The first is referred to as broker cost rate while the second as the user cost rate. Given the value of $c \_p u b$, the value of $c \_p v t$ must be chosen carefully, in order to ensure broker profit while saving user cost. If the value of $c \_p v t$ is too 
low, situations of the broker incurring a loss may arise. Since the total user cost incurred by the client enterprise is also the earning for the intermediary enterprise provider (broker), the broker profit is calculated using the following equation:

$$
\text { Broker profit }(\mathrm{BP})=\text { Total User Cost }(\mathrm{UC}) \text { - Broker Cost }(\mathrm{BC})
$$

Here, $\mathrm{BC}$ is the cost incurred by the broker for acquiring resources from the public cloud provider. The resource cost for running the auto-scaling algorithms is assumed to be negligibly small in comparison to the cost of the resources acquired for running the user requests. As indicated earlier, in addition to broker profit the research also concerns cost savings for the users. For a given set of $k$ requests with service times $\left\{c_{1}, c_{2}, \ldots, c_{k}\right\}$ seconds, the total cost savings is calculated as:

$$
\text { Total Cost Saving }(\mathrm{CS})=\sum_{i=1}^{k}\left(\left\lceil\frac{\boldsymbol{c}_{i}}{3600}\right\rceil \times \boldsymbol{c}_{-} \boldsymbol{p} \boldsymbol{u} \boldsymbol{b}-\boldsymbol{c}_{\boldsymbol{i}} \times \boldsymbol{c}_{-} \boldsymbol{p} \boldsymbol{v} \boldsymbol{t}\right)
$$

The total cost savings are derived by the summation of the cost savings for each request sent by the user by determining the dollar amount saved by the user for a request that is serviced by the broker in comparison to the broker's charge to the cloud provider for executing the same request. The first term is the amount the broker pays to the cloud provider for acquiring the resource, which is the same as the amount the user would have to pay if they rented the resource directly from the cloud provider. The second term is the amount the user pays to the broker for executing the request. Thus, the summation of the difference between the two terms for all requests is equal to CS. Note that \lceil\rceil denotes the ceiling function that rounds off a real value to its closest highest integer. Using the ceiling function is integral as the public cloud provider charges per hour of resource usage. Also, note that the broker allows multiple requests to share the same resource. The client enterprise saves costs by paying only for the time (in seconds) the resource was used for. 
It is assumed that the pubic cloud providers used in this analysis usually charge per hour [51] which requires the users pay for the full hour regardless of their actual usage, even if it is for only a few minutes. Other cost models used by the cloud service provides are discussed elsewhere in Section 8.2.

\subsection{Description of Other Systems}

Different systems are used in the analysis of cost and profit presented in the later chapters. These are briefly described in this section. The proposed system, incorporating the proposed algorithms also referred to as System I in this research, is compared with other alternative systems such as that described in [27]. The authors of [27] describe a greedy approach that acquires resources as and when required. This system is modified by incorporating a mechanism to remove idling resources for improving its performance. This modified system uses a broker that scales up when it needs an additional resource and conversely scales the VPC down if there are no requests pending execution on a particular resource. This enables the broker to increase its profits by ensuring idling resources are returned to the service provider. This system is called System II. In the system referred to as System III, users get the resources directly from the public cloud provider without going through any broker intervention. With this system, the users incur the additional overhead of starting and stopping resources, as well as handling the matchmaking and scheduling operations. For System III, only total user cost is compared with those achieved with System I and System II, as no broker is actively involved, hence it not being possible for broker profit to be accrued.

The performance of the three systems is compared with one another. The thesis also proposes a hybrid auto-scaling technique, which combines the benefits of the reactive and 
proactive auto-scaling techniques (see Chapter 6). The hybrid auto-scaling system is then compared to a purely proactive system (discussed in Chapter 5), and a purely reactive system discussed in Chapter 4. Apart from performing the prediction for the proactive system, the research introduces the GoS as it is used in the hybrid as well as the reactive system. The proactive system is referred to as System I-P and the reactive system as System I-R. The hybrid system is referred to as System I-H. The system that handles both compute and network resources is referred to as System I-RN and is discussed in Chapter 7. Both user cost and broker profit are compared for all four systems: System I-R, System I-P, System I-H and System I-RN. The various systems have been summarized in Table 3.1.

Table 3.1 Summary of Systems

\begin{tabular}{|l|l|}
\hline System I & $\begin{array}{l}\text { The system proposed in this research and represented as one of System I- } \\
\text { R, System I-P, System I-H or System I-RN }\end{array}$ \\
\hline System II & $\begin{array}{l}\text { An alternative system with a broker which is used to compare with the } \\
\text { proposed system }\end{array}$ \\
\hline System III & $\begin{array}{l}\text { An alternative system used to compare with the proposed system that } \\
\text { acquires resource directly without using a broker }\end{array}$ \\
\hline System I-R & The proposed system running a reactive auto-scaler \\
\hline System I-P & The proposed system running a proactive auto-scaler \\
\hline System I-H & The proposed system running a hybrid auto-scaler \\
\hline System I-RN & $\begin{array}{l}\text { The proposed system running a reactive auto-scaler that also handles } \\
\text { network resources along with compute resources }\end{array}$ \\
\hline
\end{tabular}




\section{Chapter 4 The Reactive Auto-scaling Technique}

This chapter focuses on reactive auto-scaling. The reactive auto-scaling system described in this thesis includes an auto-scaling algorithm that is run on every request arrival. The algorithm decides whether (i) to accept the incoming request (by invoking MMS) and consequently, (ii) to acquire an additional resource to satisfy the request. Upon acceptance of a request, it must be completed by the deadline included in the SLA. This chapter describes the auto-scaling algorithm and components of the framework that implements the reactive auto-scaling algorithm. Section 4.1 describes the auto-scaling framework while the reactive auto-scaling algorithm is described in Section 4.2. The experimental setup for performing the performance analysis of this auto-scaling technique is presented in Section 4.3 while the workload and system parameters for the experiments are described in Section 4.4. Next, in Section 4.5, the results of performance analysis for the auto-scaling framework are presented. The insights into system behaviour and performance are presented in Section 4.6.

\subsection{Broker Architecture}

Figure 4.1 outlines the different components of the broker. The request handler and MMS have been described in Sections 3.1.3 and 3.1.4, respectively. This section describes the operations performed by DM as well as the GoS module.

As explained in Section 3.1.1, the broker operates in the intermediary enterprise (IE) and has access to the resources of a public cloud. The Request Handler (RH) is the component responsible for handling user requests. It can be implemented as a web service endpoint where users can send their requests to process. The decision of whether the request is accepted or rejected is communicated back to the user. Initially, RH forwards the 
newly arrived request to DM which decides whether to accept or reject the request based on two operations:

- DM sends the request to the MMS component responsible for matchmaking and scheduling the request.

- If MMS cannot schedule the request, DM then invokes the auto-scaler component.

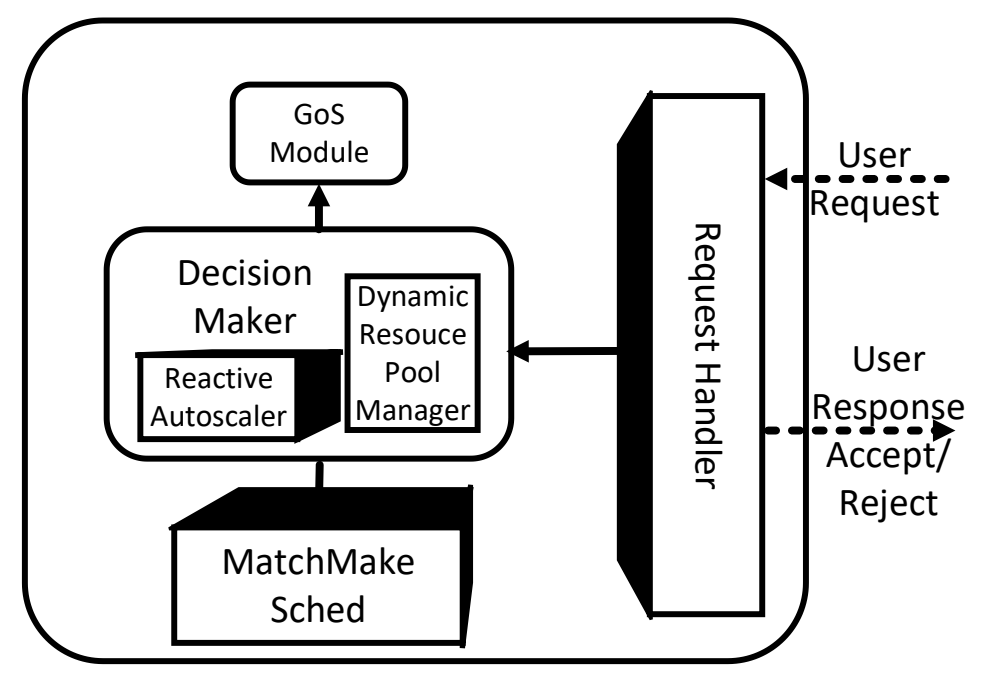

Figure 4.1: Reactive Broker Architecture

If the request is accepted by MMS, it is scheduled to be executed on the selected resource at the time computed by MMS. However, if the request cannot be scheduled in the existing resources by MMS, DM invokes the Reactive Auto-scaler (RA) to decide whether the request would be profitable for the broker. For the $i^{\text {th }}$ request having a service time of $\mathrm{c}_{\mathrm{i}}$ seconds, RA checks it against the following condition:

$$
B P_{i}>0
$$

Where $B P_{i}$ is defined as:

$$
B P_{i}=\boldsymbol{G} \times\left(\boldsymbol{c}_{\boldsymbol{i}} \times \boldsymbol{c}_{-} \boldsymbol{p} \boldsymbol{v t}\right)-\left(\left\lceil\frac{\boldsymbol{c}_{\boldsymbol{i}}}{\mathbf{3 6 0 0}}\right\rceil \times \boldsymbol{c} \_\boldsymbol{p u b}\right)
$$

Here, $G$ is a constant greater than 1 . This constant allows the reactive broker to account 
for additional requests including time-sharing the resources amongst various requests. Since the broker operates by sharing the acquired resources among other requests, $G$ is introduced to account for the possibility of the resource generating a higher profit from other requests in the time remaining before the resource is released back to the cloud provider. The broker does not allow the value of $G$ to change once the auto-scaling system is operational, and the value of $G$ remains constant for all requests.

For example, consider a situation in which the $i^{\text {th }}$ request has a service time of 1800 seconds. Also, consider the case in which the average amount of time a request executes on a resource, obtained by averaging the run times of all requests on each resource per hour, is 2520 seconds. Without using $G$, when deciding on whether to accept the request and acquire a new resource, the service time of 1800 seconds is used. This may lead to the rejection of the request, since the broker may incur a loss at this stage of the computation. However, using a value of $G$ of 1.4 (that is equal to 2520/1800) may allow the request to be accepted, because $B P_{i}$ will be greater than 0 . Since the broker operates by sharing the acquired resources among other requests, $G$ is introduced to account for the possibility of the resource generating a higher profit for the broker by the arrival of other requests in the time remaining before the resource is released.

As explained in Section 3.1, DM inside the broker controls $N$ resources at a given point in time, where $N$ changes dynamically with user demand. Once requests have been scheduled, each new resource acquired by the system has the following characteristics:

- $\quad$ Start time for the $j^{\text {th }}$ resource $\left(\right.$ start $\left._{j}\right)$ -

$\circ$ Start $_{\mathbf{j}}=\left(\mathrm{EST}\right.$ of the request expected to execute earliest on the $\mathrm{j}^{\text {th }}$ resource) $-T$ seconds 
- Note that resources are not made available immediately after a request is submitted by a public cloud provider and the additional $T$ seconds is for handling the time to start a resource by the cloud provider. This is reflected in the variable $T$. Our observation with Amazon's EC2 was that that the service provider took 120 to 240 seconds to start a resource for t 2 type instances. Generally, researchers agree that provisioning compute instances may take up to 15 minutes [8].

- $\quad$ Stop time for the $j^{\text {th }}$ resource $\left(\right.$ stop $\left._{j}\right)-$

$$
\bigcirc \quad \text { stop }_{\mathbf{j}}=\mathbf{s t a r t}_{\mathbf{j}}+\left\lceil\frac{\text { numSeconds }}{3600}\right\rceil
$$

where numSeconds is the difference in seconds between the expected completion time of the last request on resource $j$ and $s t a r t j$. Since a typical public cloud provider charges by the hour, the resource is held until the end of this "paid hour" period. Each resource $j$ has an internal timer which is set to expire at the time stop $_{j}$.

Acquiring and releasing a resource is based on the following three rules:

- $\quad$ Rule I: When $B P_{i}>0$, acquire $j^{\text {th }}$ resource

- $\quad$ Rule II: When $\left(s t o p_{j}\right)=$ current time, release $j^{\text {th }}$ resource

- Rule III: If $B P_{i}<0$ and rejecting the request would violate the GoS criterion, acquire $j^{\text {th }}$ resource

Rule I states that the $j^{\text {th }}$ resource is acquired if a profit is generated for the broker. Rule II specifies the release of the $j^{\text {th }}$ resource at its stop time.

Rule III states that even if the calculated value of $B P_{i} \leq 0$, DM needs to check whether the GoS criterion is satisfied. Although the system described in this research uses the blocking ratio for describing the GoS, the framework can be adapted to other metrics as 
well. This auto-scaling technique uses the concept of auto-scaling based on broker profit in addition to the GoS criterion described in Section 3.1.1.

DM consults with the GoS module that contains configuration details. The desired value of $B$ is specified by the user beforehand and is denoted by $B_{\text {spec }}$. As described in Chapter 3, $B$ denotes the current ratio of requests rejected by the system to the total number of requests received by the system and is continuously monitored on the system. Irrespective of whether a profit will accrue, a resource is acquired if:

$$
B>B_{\text {spec }}
$$

Rule II may force the broker to accrue a loss for a request that needs to be accepted to keep $B_{\text {spec }}$ at the desired level and maintain the GoS criterion. This may happen when the arrival rate of user requests as well as the service time of the user requests is low. Additionally, a value of $B_{s p e c}$ close to 0 may force the broker to acquire new resources for every request and that may lead to a loss for accepting requests with low job execution times.

\subsection{The Reactive Auto-scaling Algorithm}

The reactive auto-scaling algorithm, as outlined in Table 4.1 is described next. For every incoming request $R e q_{i}$, the algorithm is executed. The algorithm only describes the scale up operation. Resources are scaled down automatically when the current time becomes greater than the stop time as described in Rule II in Section 4.1. The stop times are set in two cases, when a new resource is started or when an existing resource is determined to be profitable for a new request and the stop time is extended. Acquisition of resources suggested by DM follow Rule I. Lines 1- 7 use the MMS algorithm to determine whether the existing set of resources can accommodate the new request. DM sends each 
request to MMS which subsequently decides whether one of the resources in the current set of resources can be allocated to meet the request deadline. If the request cannot be accommodated in the existing set of resources, the auto-scaling algorithm is executed to determine whether a profit may accrue from the request after acquiring a new resource from the public cloud provider. An exception to this case is when the value of $B$ goes above the specified GoS condition $\left(B_{\text {spec }}\right)$. In this case, the resource is acquired, irrespective of whether a profit is obtained by scheduling the new request on a newly acquired resource. Although the broker may accrue a loss occasionally, the results of experiments show a significant overall profit for the broker for the various system and workload parameters experimented with. The process of resource acquisition is shown in lines $8-14$. The start and stop times of the resource to be acquired are set in line 12 and line 13 respectively. Line 14 performs the action of acquiring the new resource. Resources are released when the current time reaches the stop times, as computed in line 13 and stored in their respective timers.

Table 4.1 Reactive Auto-Scaling Algorithm

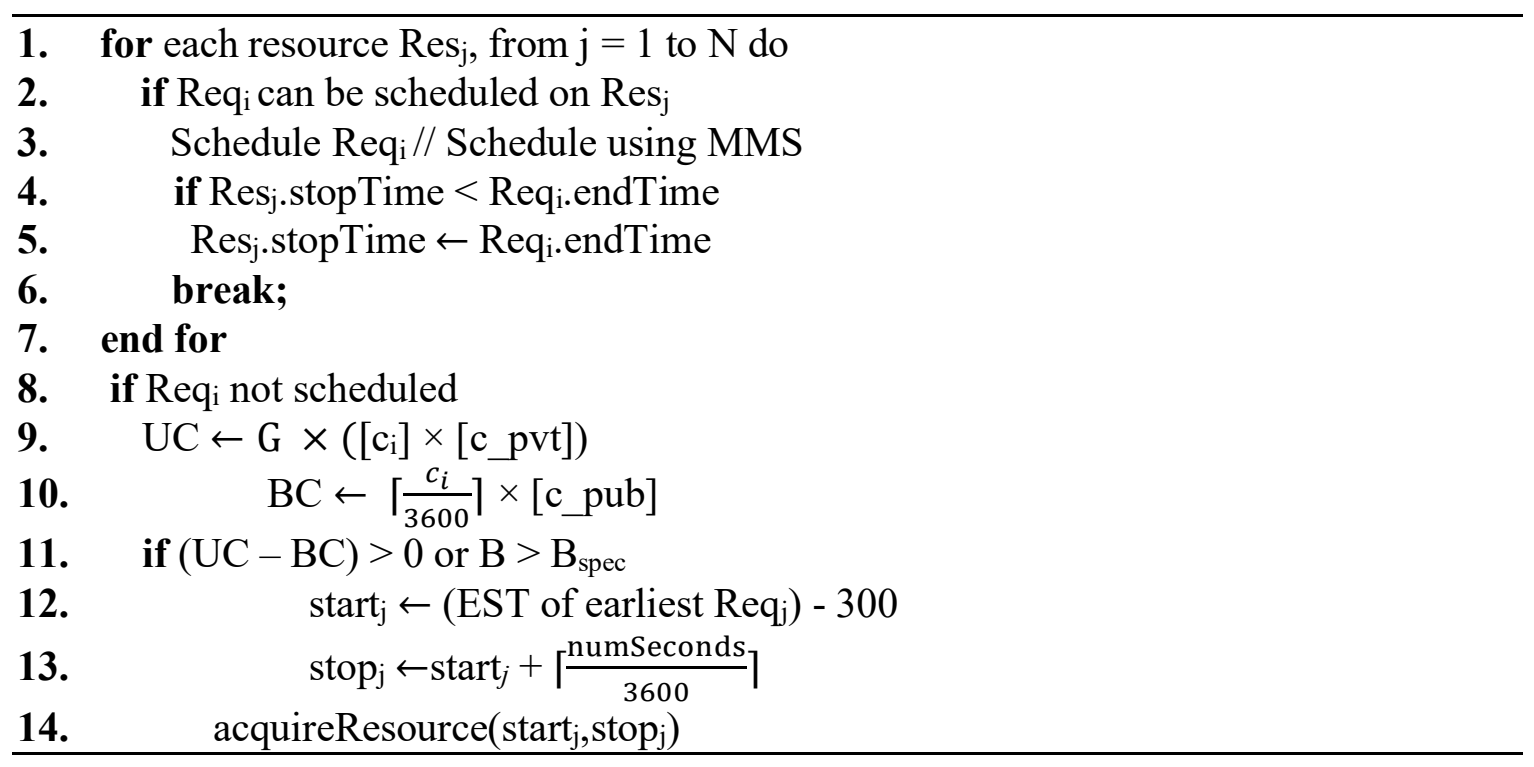




\subsection{Experimental Setup}

This section describes the experimental setup. Section 4.3.1 describes the programming language and framework used in the implementation of the prototype. Section 4.3.2 describes the details about the implementation and challenges faced while deploying the broker on a real cloud environment.

\subsubsection{Programming Language/Framework}

The experimental platform is implemented using the Java programming language. Representational State Transfer (REST) [52] is chosen as the architectural pattern for designing the distributed framework. The system needs two different communication operations:

- Communication between the client enterprise, SCE and the broker.

- Communication between the broker and the resources in the private cloud.

The messages transferred using the REST architecture are in the form of the JavaScript Object Notation (JSON) [24]. JSON syntax is used for storing and exchanging data which is easier to use alternative to Extensible Markup Language (XML). In comparison to XML, JSON is much easier for humans to read or write. Machines also parse JSON faster.

The framework works with different cloud platforms such as Amazon Web Services as well as OpenStack. DRPM, one of the components of the broker, uses a Software Development Kit (SDK) to communicate with each of these two cloud providers. Other cloud providers can also work with this system, provided DRPM has the correct SDK to invoke resource creation operations as well as the correct API keys that allow DRPM access to the cloud provider's resources. More details of DRPM are provided in Section 3.1.5. 
The framework uses Spring [53] as the underlying framework to achieve loose coupling between different components. Spring Context is used for providing the dependency injection design pattern which removes hard-coded dependencies and make the system loosely coupled, extendable and maintainable. The application also uses the Spring Web Module to create web components. The application exposes REST end points as web services to help communication among the internal components of the broker as well as between the broker and SCE and the broker and VPC. Gradle [54] is used for building and deploying the application. It is written as a Groovy script. Gradle also helps to support external dependencies using Maven [55]. The external projects used by this application are listed:

- $\quad \log 4 \mathrm{~J}$ - a Java-based logging utility.

- Date4J - a Java-based date library for working with nano-second time precision, used in all modules that depend on measuring time.

- $\quad$ SSJ - a Java based stochastic simulation library for generating random numbers and distributions.

- $\quad$ AWS - an SDK by Amazon for controlling resources in the Amazon Cloud.

- OpenStack4j - an SDK by OpenStack for controlling resources in the OpenStack Cloud.

Initially, a simulator for the same algorithm was created. One of the main benefits of the simulation framework is that experiments may be completed in a lesser amount of time as compared to the prototype implementation. This is due to the simulator not waiting for requests to finish execution and possessing the ability to skip to a point where a request has finished its execution. This is an event-driven simulator that simulates the resources of the 
public cloud by building a mock interface similar to the cloud API used by OpenStack and Amazon Web Services. More details about the simulator may be found in Section 4.4.

\subsubsection{Implementation}

This research is based on dual implementation; one with a prototype implementation using resources from a real cloud provider and the other using a simulation as explained in Section 4.3.1. For the prototype, synthetic requests with a desired set of request attributes are generated on a desktop computer outside the cloud environment. This computer acts as a source for all requests from the client enterprise and is equipped with an Intel Core i7, 4 CPUs (2.8 Ghz) and a $12 \mathrm{~GB}$ of RAM. Each request is sent to a broker running on the public cloud provider using REST. After generating a single request, this user module waits for the given inter arrival time duration before generating a successive request. The request characteristics such as earliest start time, service time and deadline are generated using the respective distribution discussed in Section 4.4.1

When the broker receives a new request via $\mathrm{RH}$, it uses MMS to determine whether the request can be scheduled on one of the resources available to the broker. Scheduling and matchmaking of the request is performed as explained in Section 3.1.4. The decision to accept or reject a request is sent back to the user using the REST framework. If MMS allows the request to be scheduled, the broker sends the request via REST to the chosen resource, where it is queued. The matchmaker selects the first resource in its queue. The broker starts executing the request after its earliest start time. The execution of a request is emulated by running a for-loop to keep the CPU busy for the predetermined service time duration of the request. Once the request finished execution on the resource, the resource informs the broker of the request completion. 
Both the simulation environment and the prototype are tested with various combinations of system and workload parameters. More details about the prototype and the simulator are found in Sections 4.3.2.1 and 4.3.2.2, respectively.

\subsubsection{Prototype}

The resources in the prototype are acquired from the Amazon's EC2 cloud service. The broker ran inside the EC2 cloud on a $2 . s m a l l$ instance. This instance is kept in a public subnet accessible to the Internet. The rationale behind this is to allow clients to communicate with the broker without any restrictions. If the broker was kept inside the VPC without direct access to the internet, communicating between SCE and the broker would be severely restricted. All ports except 8080 are closed to only allow RH, running as a web service, to be available to SCE. The resource pool, controlled by the broker using DRPM, is placed in a private subnet without Internet access. Each resource was running on an Amazon t2.micro instance.

Running experiments with a prototype required compute resources from Amazon to be acquired and released. In order to complete each experiment in a reasonable amount of time and to reduce the costs required to rent resources from Amazon, the run time for the experiments were scaled down by a scaling factor of 300 approximately. In order to achieve this scaling factor of 300 a request inter arrival time of 450 seconds, for example, was scaled down to an inter arrival time of 1.5 seconds. Similarly, an execution time of 3000 seconds would result in the scaling down of the execution time of the request to 10 seconds. When running experiments, the requests were generated by scaling all request characteristics to their appropriate time durations. Once requests were scheduled on a resource, after the start time of the request, the compute resource was kept busy executing 
the request for the scaled value for the service time specified in the request characteristics.

\subsubsection{Simulator}

For the simulator, the properties of the resources are stored in a java class file and any request submitted to the resource would be queued until the start time of the request. This would be similar to the scheduler on each resource handling a set of requests scheduled to be executed on the specific resource. For the simulation, instead of sending the requests to a broker running on the cloud, they are sent to a broker which is running on the same computer as the one where the requests were being generated from. The resources are simulated inside the same computer.

\subsection{Experimental Parameters}

This section presents the different values for parameters used in experiments to evaluate the performance of the reactive auto-scaler with other comparable systems. The values for each parameter used are given in Table 4.2 and Table 4.3. Each experiment is performed by changing one parameter at a time while holding the other parameters at their default values. Column 2 specifies the set of all values used for the respective parameter. The default values are shown in boldface

\subsubsection{Workload Parameters}

Similar parameter values have been used by other researchers [47] [56]. In these experiments, $20 \%$ of the requests sent by the user were OD requests while the remaining were AR requests. Workloads comprising of a higher and lower proportion of OD requests are observed to lead to a similar set of conclusions. While systems having a single mean arrival rate have been typically studied in the literature, in some systems $\lambda$ can vary, based on the time of the day or day of the week. In addition to a system subjected to a single 
arrival rate, a system subjected to two arrival rates $\left(\lambda_{\text {low }}=0.0027\right.$ requests $/$ second and $\lambda_{\text {high }}$ $=0.0053$ requests/second) was also experimented with.

Each simulation was run long enough such that the system operated in a steady state. The total number of requests processed by the broker for each experiment has been kept at 6000 requests, which allows the system to reach a steady state. Each experiment was repeated a sufficient number of times such that an interval less than or equal to $\pm 5 \%$ was achieved at a confidence level of $95 \%$ for each performance metric. The confidence interval was computed through a Python script that used the method outlined in [57] for computing the statistics related to confidences intervals. At the end of each experiential run, these statistics were computed, and additional runs were performed if the desired levels of these statistics were not achieved. The simulation model comprises of a workload model and a system model. The parameters of the workload model are presented first followed by the parameters of the system Model.

Load Factor $(f)$ - is the ratio of the number of requests generated with an arrival rate of $\lambda_{\text {low }}$ to the total number of requests generated during the experiment. Thus, if the number of total requests is $k$, then the number of requests with an arrival rate $\lambda_{\text {low }}$ is $f \times k$ and the number of requests with arrival rate $\lambda_{\text {high }}$ is: $(1-f) \times k$.

Mean Service Time $(S)$ in minutes - characterizes the execution times of the requests that are uniformly distributed [56].

Earliest Start Time (EST) - The earliest start time for the request is computed by adding a value $V$, which is uniformly distributed between 0 and 12 hours, to the arrival time of the request. Other researchers have used similar distributions [29] [47] for similar request types. 
Deadline $(\boldsymbol{D L})$ - The deadline for the request is computed as:

Deadline for a request $(D L)=$ Earliest Start Time of request + Request Service Time + Laxity

Laxity - is defined as the difference between the deadline of the request and the time duration of the service time of the request added to the earliest start time.

Laxity $=[$ Deadline $-($ Earliest Start Time $)-$ Service Time $)]$

Laxity is also known as slack time of a request, or the time the request has in addition to its service time before it reaches its deadline.

Laxity Factor $(\boldsymbol{L} \boldsymbol{f})$ - is an integer characterizing the slack time of requests.

Laxity Factor $=($ Laxity $\div$ Request Service Time $)$

Mean Arrival Rate $(\lambda)$ in requests per second - is the rate at which requests arrive on the system. A Poisson distribution is used to model the request arrivals [56].

Table 4.2 Summary of Workload Parameters for Reactive System

\begin{tabular}{|c|c|}
\hline Load Factor: $\boldsymbol{f}$ & $0,0.2, \mathbf{0 . 4}, 0.6,0.8,1$ \\
\hline Mean Service Time (in mins): $\boldsymbol{S}$ & $\mathbf{5 0}$ \\
\hline Laxity Factor: $\boldsymbol{L} \boldsymbol{f}$ & $0, \mathbf{1}, 2,3,4$ \\
\hline Mean Arrival Rate (in reqs/sec): $\boldsymbol{\lambda}$ & $0.0083,0.0067, \mathbf{0 . 0 0 5 3}, 0.0037, \mathbf{0 . 0 0 2 7}$ \\
\hline
\end{tabular}

\subsubsection{System Parameters}

The default public cloud provider cost is based on current Amazon charges; 2 cents [2] for one hour of using a micro instance. The constant $G$ is fixed at 1.2 and $B_{\text {spec }}$ is fixed at $20 \%$. The system parameters are given in Table 4.3 . 
Table 4.3 Summary of System Parameters for Reactive System

\begin{tabular}{|c|c|}
\hline User cost rate (in \$ per sec): $\boldsymbol{c} \boldsymbol{p} \boldsymbol{v \boldsymbol { t }}$ & $0.00000694, \mathbf{0 . 0 0 0 0 0 8 3 3}$, \\
\hline Broker cost rate (in \$ per hour) for a micro & $0.00000972,0.0000111$, \\
instance: $\boldsymbol{c} \boldsymbol{p} \boldsymbol{u} \boldsymbol{b}$ & $0.0000125,0.0000139$ \\
\hline Constant: $\boldsymbol{G}$ & 0.02 \\
\hline Specified Probability of Blocking: $\boldsymbol{B}_{\text {spec }}$ & 1.2 \\
\hline
\end{tabular}

\subsubsection{Performance Metrics}

Broker profit $(B P)$ : is the profit a broker earns per hour in dollars.

Total User cost $(U C)$ : is the amount in dollars charged per hour to the user.

A higher $B P$ and a lower $U C$ indicates good system performance.

\subsubsection{System Overheads}

The average overhead of an operation is characterized as the average time spent by all the requests during an experiment on performing the given operation. The overheads considered are presented:

Average Overhead due to Internal Communication: corresponds to the delay in communication between the resources and the broker. This overhead accounts for the difference between the time at which the response to a message is received by the broker and the time at which the request is sent from the broker to a resource. The average overhead is calculated by aggregating all the delays in the message communication between the broker and the resource and dividing it by the number of such delays that 
occurred in the experiment.

Average Overhand due to External Communication: corresponds to the delay in communication between the user and the broker. Initially the user sends the request to the broker. This overhead accounts for the difference between the time at which the response to a message is received by the user and the time at which the request is sent from the user to the broker. The average overhead is calculated by aggregating all the delays in the message communication between the broker and the user and dividing it by the number of such delays that occurred in the experiment.

\subsection{Results}

This section presents the results obtained by simulation framework. In addition to the simulator, a proof of concept prototypes for System I-R, System II and System III was built and run on a system using Amazon EC2 as the public cloud. System II and System III have been described in Section 3.3. The results show that the prototype results are similar to the results obtained through the simulation. In addition, these experiments have $20 \%$ of the requests sent by the user as OD requests while the remaining are AR requests. The value of $G$, which is introduced in Section 4.1, when calculating the profit function, is kept at 1.2. This considers the possibility of additional requests arriving to use the idle time for the resource after the completion of the request that caused the resource to be acquired. The effect of using other values of $G$ was also evaluated. Initially, $B P$ was observed to increase with an increase in $B$. It was observed that increasing $G$ from 1 to 1.2 resulted in a $7 \%$ increase in $B P$. However, increasing of $G$ beyond 2 did not result in any significant increase in $B P$. This gives a $20 \%$ probability of a new request arriving to use the remaining time on the resource. $B_{s p e c}$ is maintained at $20 \%$ which guarantees that at least $80 \%$ of the 
user requests will be executed. Given the $B_{\text {spec }}$ maintained by the system, auto-scaling operations are effective in matching the workload demand by provisioning adequate capacity, as demonstrated in Section 4.5.1.

\subsubsection{Demonstration of Auto-scaling Performed on the System}

The expected relationship between capacity and workload demand is captured in Figure $1.2(\mathrm{c})$, which shows the ideal relationship: capacity always changes to exactly meet the demand. The relationship between the number of active requests that approximates demand and capacity represented by the number of compute resources in the system is presented for the reactive auto-scaler in Figure 4.2. Note that since the proportion of rejected requests B for the experiment is very small $(0.03)$ the number of active requests at any given time (hour number shown on the x-axis) is very close to the incident workload demand that is captured by the number of requests that have arrived on the system in the recent past. Figure 4.2 presents the relationship between the number of active requests and the number of resources on the system by plotting the data observed on the hour for a 25hour period obtained by running the simulation experiment for the default set of system and workload parameters. The plot shows a close agreement: for most of the time instances: the number of resources is either equal to or slightly higher than the number of active requests. The figure demonstrates the effective auto-scaling performed on the system: an increase or decrease in the number of active requests is accompanied by a corresponding increase or decrease in the number of resources. The trend shown in Figure 4.2 has also been observed when using the proactive or the hybrid auto-scaling approaches. 


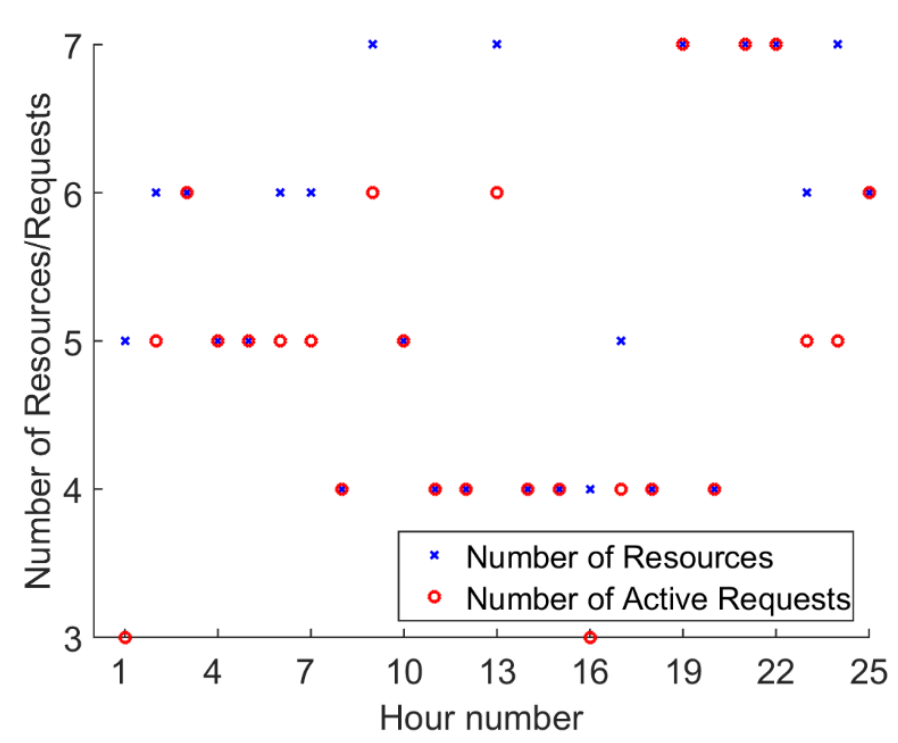

Figure 4.2: Relationship between the Number of Resources and Number of Active Requests for the Reactive System

\subsubsection{Comparison between the Simulator and the Prototype}

On comparing the simulator and prototype results, a close match was found between the measurement results from the scaled prototype and the simulation results. Figure 4.3 presents a comparison of $B P$ with $L f$. The profit is inversely proportional to $f$ : as $f$ increases, profit increases. This is because as $f$ increases, the proportion of requests with lower arrival rate increases. Consequently, on average, the broker has a fewer number of requests to process per unit time, causing the broker profit per hour to decrease. In most cases, a difference of $2 \%$ to $5 \%$ was observed with a largest difference of $6 \%$ between the simulator and the prototype with the simulator performing better than the prototype. The better performance of the simulator may be attributed to the assumption of zero communication latencies whereas the communication between the broker and the virtual resources as well as between SCE and the broker in the prototype are subject to non-zero communication latencies with network latencies due to Amazon's internal data center 
network and external networks having a small impact on broker performance for the prototype. The latencies for communication between the broker and the resources in the simulator are very small.

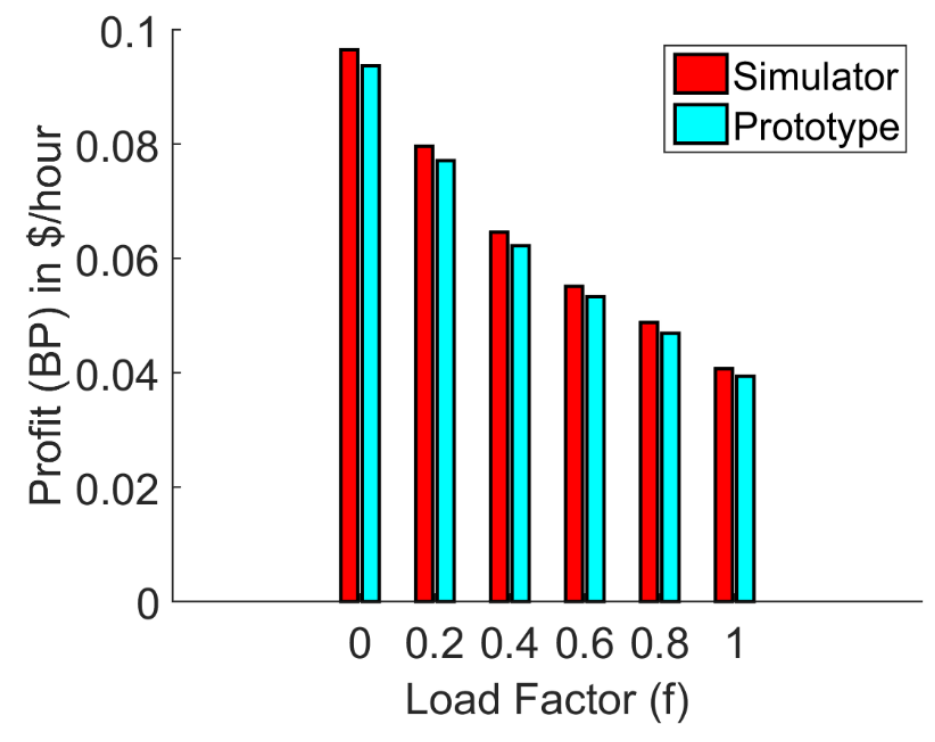

Figure 4.3: Comparison of the Simulator and Prototype for the Reactive Auto-scaler

Since the difference in the results for the simulator and the prototype is small, the remaining experiments in this chapter have been run using the simulator, thereby saving costs associated with acquiring resources on a public cloud as well as saving time to run the experiments in the cloud environment. Note that the system overheads reported in Section 4.5.8 are based on measurements made on the system prototype. Additionally, a close match between the prototype and the simulator results was found for System II as well, and hence only the simulator results for System II have been presented in this chapter.

\subsubsection{Impact of Load Factor}

Figure 4.4, compares the profit that accrues on System I-R and System II for different values of $f$. Note that the profits that are accrued by the broker are dependent on the request arrival rate. Higher profits will be accrued by a larger system that is subjected 
to higher arrival rates. In all cases, for a given $f$, System I-R is able to generate more profit than System II. As seen in Section 4.5.1, the profit is inversely proportional to $f$ : as $f$ increases, profit increases. For example, when $f$ is $0, B P$ is the highest. Note that at $f=0$, the system is subjected to only the higher arrival rate. As $f$ increases, the proportion of time for which the system is subjected to the lower arrival rate increases. At $f=1$ the system is subjected to only the lower arrival rate and the profits earned are lower. Overall, for a given $f, B P$ for System I-R is 3 - 4 times higher than System II.

Figure 4.5 presents a comparison of the total user cost incurred in all the three systems. As shown in the figure, for a given $f$, System I-R leads to a lower total user cost in comparison to the other two systems. All requests are considered to be arriving from a group of users that belong to SCE. As a result, an increase in arrival rate implies a higher cost per hour for the user.

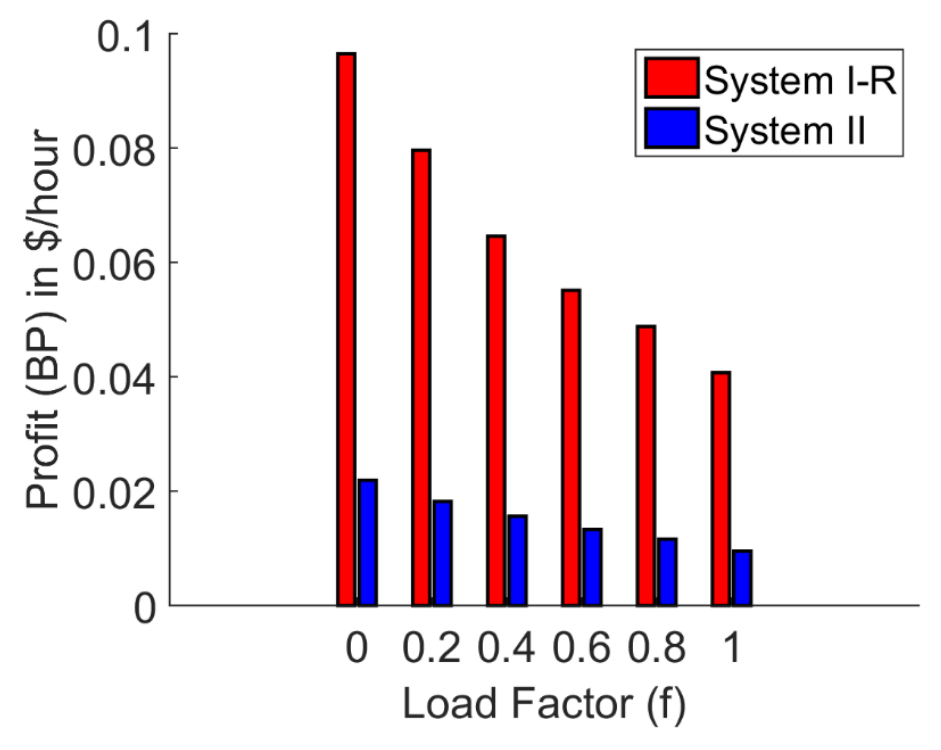

Figure 4.4: Effect of $f$ on Broker Profit for the Reactive Auto-scaler 


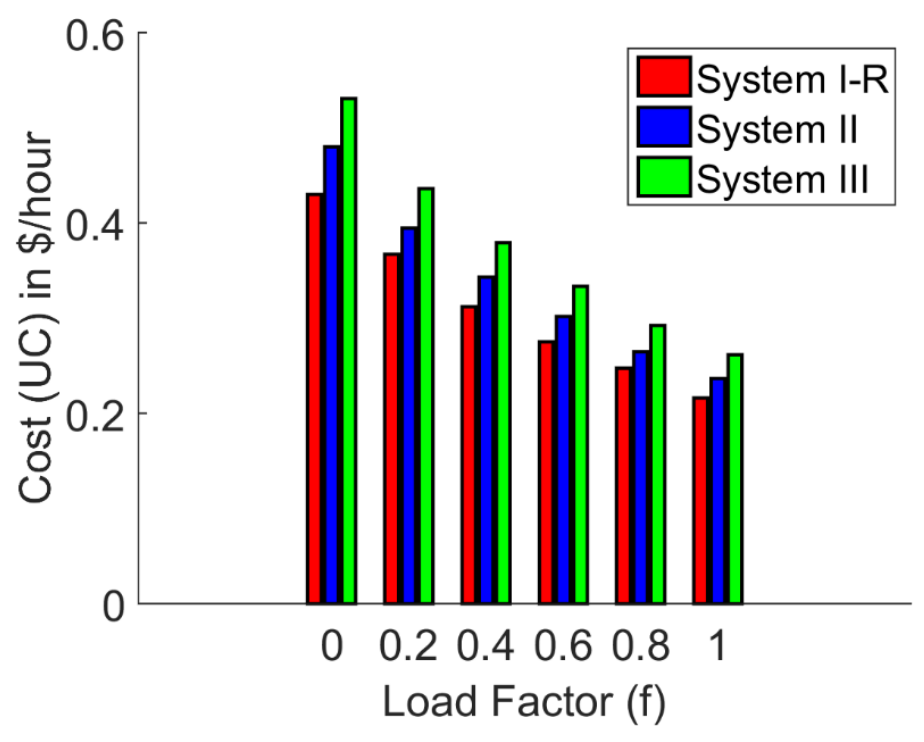

Figure 4.5: Effect of $f$ on User Cost for the Reactive Auto-scaler

\subsubsection{Impact of Arrival Rate}

A performance analysis of a system subjected to a single arrival rate is performed to investigate the impact of $\lambda$ on profit. Figure 4.6, presents the effect of $\lambda$ on the profit generated on System I-R and System II. BP in both systems is directly proportional to the arrival rate. This is because at higher arrival rates, the system receives a higher number of requests per unit time, thus increasing the potential for earning a higher profit/hour. For a given $\lambda$, System I-R earns in between 2 to 6 times the profit earned by System II.

\subsubsection{Impact of Laxity Factor}

In Figure 4.7, Systems I-R and II are subjected to different values of $L f$. A $L f$ of 0 implies that there is no additional slack time for the requests. This renders the auto-scaling technique ineffective because the system cannot schedule multiple requests without missing some of the deadlines. Consequently, the profit accrued is almost 0 . However, as the laxity in the system increases, $B P$ also increases. A $150 \%$ improvement in $B P$ is observed as the $L f$ is increased from 1 to 4 . Overall, for a given $L f$, System I-R perform 3 
to 6 times better than System II. Another interesting observation is that as $L f$ increases from 1 to 2, there is a considerable change in $B P$.

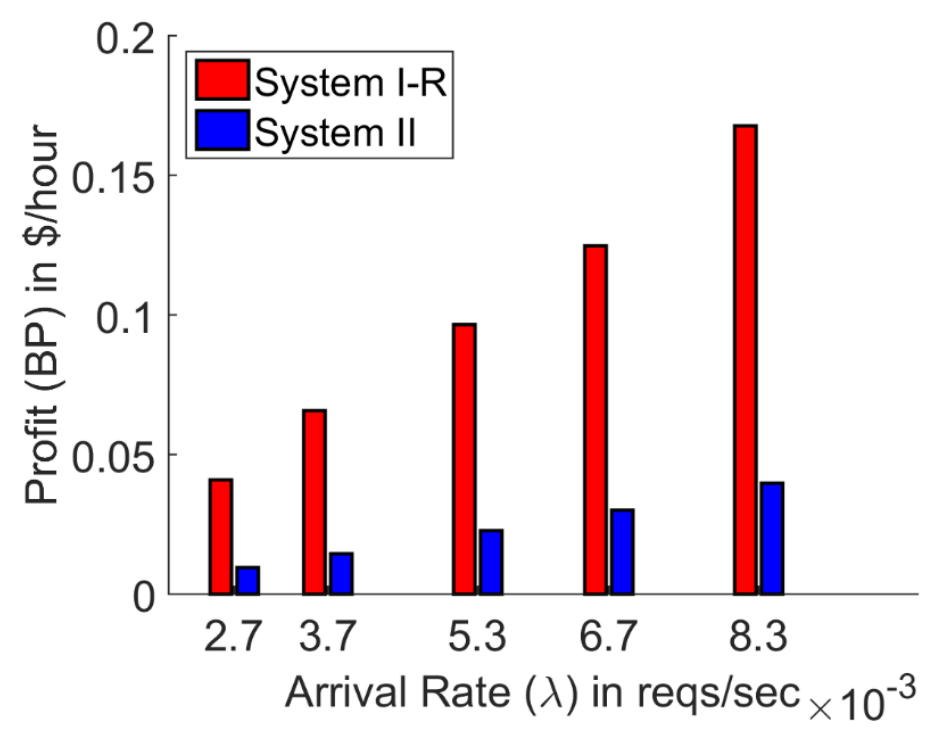

Figure 4.6: Effect of $\lambda$ on Broker Profit for the Reactive Auto-scaler

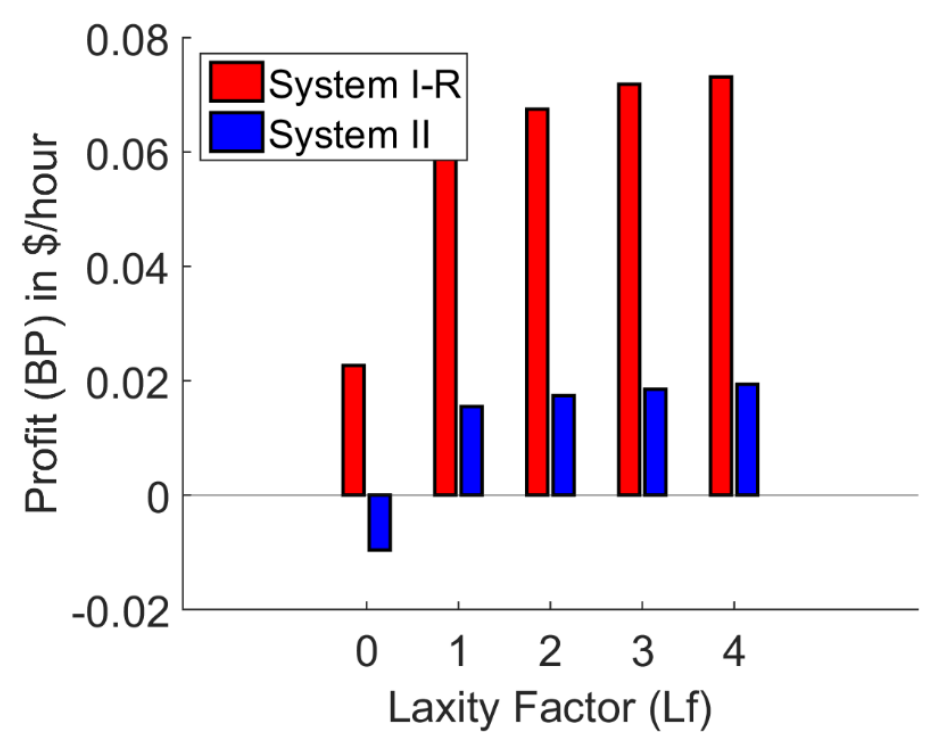

Figure 4.7: Effect of Lf on Broker Profit for the Reactive Auto-scaler

However, the increase in $B P$ seems to flatten as $L f$ is further increased. An explanation for this may be that at lower $L f$ values, the requests have stricter deadlines leading to more requests being rejected. The auto-scaler benefits significantly from an 
increase in $L f$, as the slack time for scheduling the requests is increased thus enabling the broker to schedule more requests. As $L f$ increases, most requests have a longer deadline, which makes it easier for the broker to schedule them. Having extremely large deadlines at the highest $L f$ values do not contribute to the broker scheduling more requests per hour, thus leading to an only small increase in $B P$.

\subsubsection{Impact of Service Time}

Figure 4.8 captures the impact of $S$ on the performance of System I-R and System II. The figure shows that the profit increases with an increase in $S$. This is because the larger the service time of a request, the higher the utilization of the resources. This enables the broker to exploit the pricing difference and earn a greater profit at a higher value of $S$. For a mean service time of 12.5 minutes, however, the system accrues almost no profit. Note that for the parameters used in this experiment it would take at least 40 minutes of requests running on a resource per hour for the system to break even and lead to a broker profit. This is because at the default rate of $0.00000833 \$ / \mathrm{sec}$ charged to the user by the broker, executing requests for 40 minutes earns the broker $\$ 0.02$ which is also the same amount the public cloud provider charges the broker per hour. System II follows a similar trend as displayed by System I-R. For any given $S$ between 25 and 75 minutes, broker profit for System I-R is 1.5 to 4 times that for System II. 


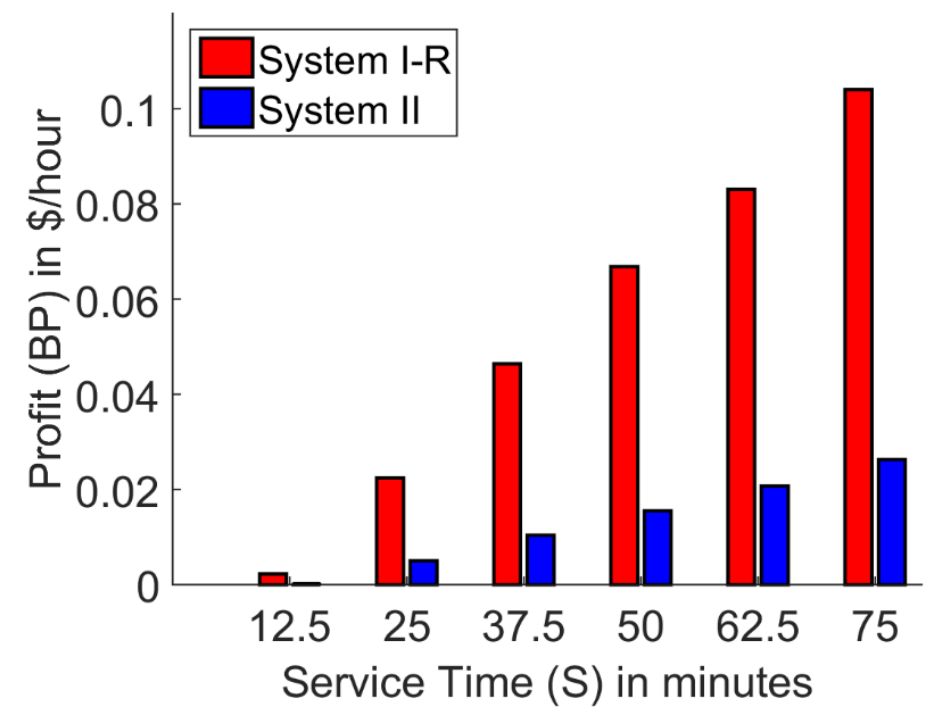

Figure 4.8: Effect of $S$ on Broker Profit for the Reactive Auto-scaler

\subsubsection{Impact of User Cost Rate}

In Figure 4.9, the effect of changing values of the user cost rate, $c \_p v t$, on $B P$ is presented for a fixed broker cost rate, $c \_p u b$, held at 0.2 dollars per hour. The user is charged between 0.00000694 and 0.0000139 cents per second. Profit is observed to increase monotonically with an increase in the user cost.

Since a higher value of the user charge generates a higher charge to the user, the profit accrued for the broker also rises. It may be noted that when the user is charged 0.00000694 , there is very little profit accrued by the broker. This is because the user effectively pays $\$ 0.025$ / hour for a resource while the public cloud provider charges the broker $\$ 0.02$ /hour. Hence, for the broker to register any profit, each instance must be kept busy for 48 minutes every hour (for the same reason why it takes 40 minutes for a charge of $\$ 0.02$ as explained in Section 4.5.6). As the user charge increases, the broker margins also increase. 


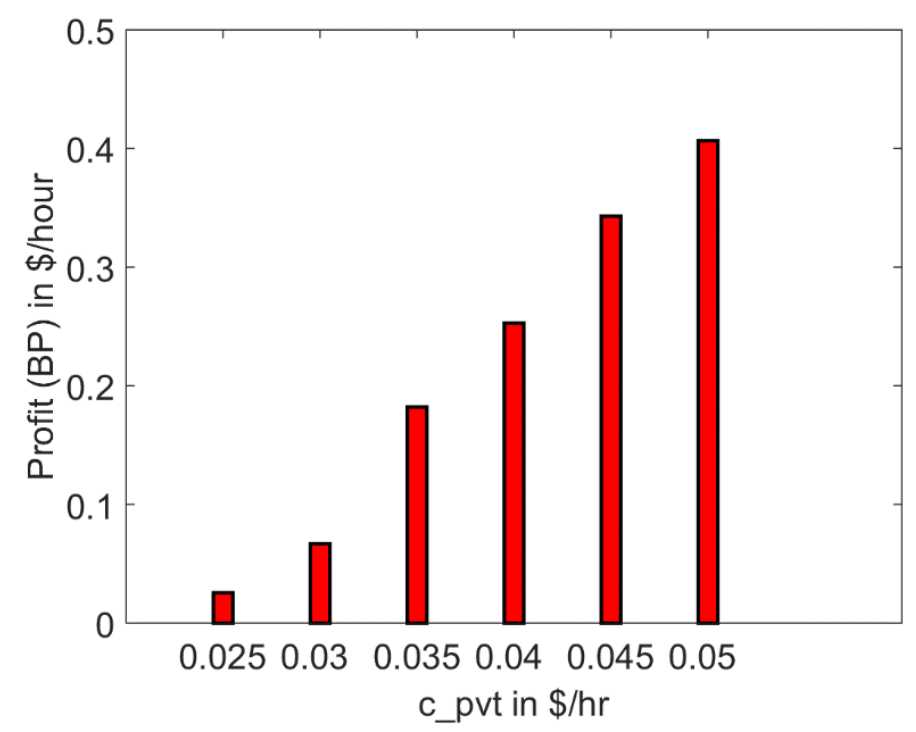

Figure 4.9: Effect of $c \_p v t$ on Broker Profit for the Reactive Auto-scaler

\subsubsection{System Overheads}

An experiment was conducted with all the system and workload parameters held at their default values. The average overhead due to internal communication (defined in Section 4.4.4) is measured to be 63 milli-seconds whereas the average delay due to external communication (also defined in Section 4.4.4) is measured at 182 milli-seconds. The overheads are very small in comparison to the mean service time of a request, which is 50 minutes, or 3000 seconds.

\subsubsection{Impact of Other factors on Total User Cost}

Table 4.4 presents some of the parameters and the highest percentage cost saving among all experiments of a particular parameter for System I-R over Systems II and III. For each experiment, System I-R led to a smaller total user cost in comparison to Systems II and III. Table IV presents the savings in cost achieved with System I-R in comparison with Systems II and III. The first column specifies the experiment by describing the parameter that was varied during the experiment. The highest improvement in total user 
cost achieved by System I-R over System II and by System I-R over System III are presented in columns 2 and 3 respectively.

Table 4.4 Highest Percentage Cost Savings of System I-R Over System II and System III

\begin{tabular}{|c|c|c|}
\hline Experiment & System II & System III \\
\hline Impact of $\lambda$ & $34 \%$ & $70 \%$ \\
\hline Impact of $S$ & $10 \%$ & $16 \%$ \\
\hline Impact of $L f$ & $8 \%$ & \\
\hline
\end{tabular}

\subsection{Observations}

As demonstrated through simulation experiments and measurements, the proposed reactive auto-scaling technique can generate a profit for the intermediary enterprise hosting the broker while reducing the cost incurred by users. The auto-scaling framework is capable of handling both $\mathrm{AR}$ and $\mathrm{OD}$ requests. The chapter also discusses the various overheads associated with the system and demonstrates that these overheads are small. A number of important features of the technique as well as key insights into system behaviour and performance obtained for the performance analysis described in Section 4.5 are presented:

- The number of resources in the pool used by the user requests need not be determined a priori and are controlled dynamically thereby reducing the cost for capacity planning.

- $\quad$ The framework allows users in an enterprise for example, to submit AR as well as OD requests to a private cloud provided by the intermediary cloud provider where resources from a public cloud are used to handle the workload. 
The framework provides a minimum grade of service to ensure that a certain percentage of user requests are guaranteed to be executed.

- The experimental results demonstrate that using the proposed reactive broker can lead to a higher profit as compared to other non-reactive systems. For example, as shown in Figure 4.8, an improvement in BP up to $300 \%$ has been observed. The broker profit is observed to depend on parameters, such as load factor, arrival rate, mean service time, laxity factor and user cost.

- A higher mean arrival rate enables the broker to earn a higher profit. As a result, lower the load factor higher is the difference between the profit generated by the reactive system and that generated by a conventional system.

- The broker profit is directly proportional to the user charge of the client enterprise and to the mean service time of the request.

- Using a per second billing rate for the user led to a lower user cost achieved by the reactive technique (System I-R) in comparison to a conventional system that rents resources directly from the public cloud provider (System III).

- A minimum of 40 minutes needs to be utilized per hour of resource use to ensure that the broker does not incur a loss. This is because the broker is charged $\$ 0.02 /$ hour by the cloud provider. The amount the broker charges the SCE for executing a request is $\$ 0.00000833 /$ second. The broker would need to schedule a request on every resource for at least 40 minutes to recover the amount it paid to the cloud provider to rent the resource for an hour. Here, the minimum time for which one or more requests must be executed on a resource to enable the broker to be profitable is dependent on the values of $c_{\_} p v t$ and $c \_p u b$. 
The total user cost for the reactive technique is observed to be inversely proportional to the load factor.

- The broker profit seems to increase with the increase in the laxity factor. Most laxity factors experimented with lead to a significant broker profit. However, with a laxity factor of 0 , the requests have no additional slack time to meet their deadlines. In such a situation, the profit earning mechanism does not work well. 


\section{Chapter 5 The Proactive Auto-scaling Technique}

The proactive auto-scaling technique deploys an auto-scaler that uses a machine learning engine (MLE) to predict requests arriving at the system in the future. The algorithm decides whether or not to acquire additional resources based on the predictions made by MLE. On every request arrival, a decision maker decides on whether to accept or reject the incoming request based on resource availability. Upon acceptance, the request must be completed by the deadline specified by the SLA. Although the proactive algorithm may reject requests occasionally, our results for the workload and system parameters experimented with show that the proportion of requests rejected by the broker in a given experiment remain below the specified GoS. This chapter describes the components of proactive auto-scaling technique in Section 5.1. The proactive auto-scaling algorithm is described in Section 5.2. The experimental setup for performing the performance analysis of the auto-scaling technique is present in Section 5.3 while the workload and system parameters for the experiments are described in Section 5.4. Next, in Section 5.5, the chapter presents the results of the performance analysis. The observations from the experimental analysis of the proactive auto-scaling techniques are summarized in Section 5.6.

\subsection{Broker Architecture}

The following sections describe the components of the broker system which is shown in Figure 5.1. The system is divided into four distinct components. The first component is the request handler $(\mathrm{RH})$ that handles user requests. The second component known as MatchMakeSched (MMS) makes the decision to accept or reject a request. The request handler and MMS were described in Section 3.1.3 and Section 3.1.4 respectively. 
The third component is the Decision Maker which manages auto-scaling operations. To decide when resources need to be scaled up, DM uses a fourth component known as Machine Learning Engine. MLE uses past workloads to predict future request arrival patterns. The final component is the GoS module, which maintains the grade of service. This section describes the operations performed by DM as well as MLE and the GoS module.

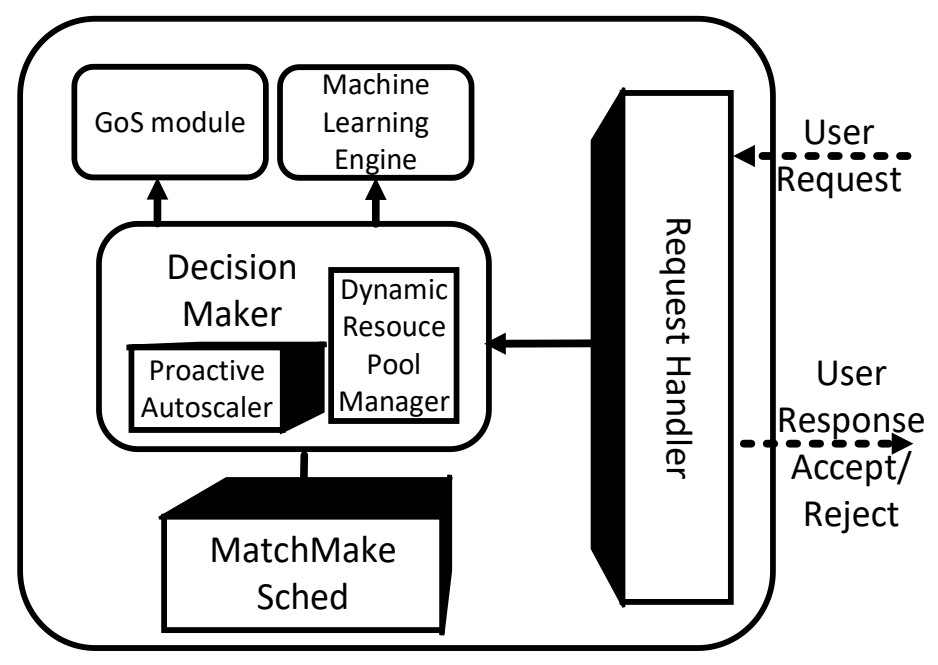

Figure 5.1: Proactive Broker Architecture

\subsubsection{Operations}

The broker that is a part of IE is operated as a service on the public facing side in the public cloud environment as part of IE. Users from a single client enterprise send their requests to the broker which utilizes resources from the private cloud to satisfy the requests. All the incoming requests are first directed to RH. As described in Section 3.1.1, the broker controls $N$ resources at a given point in time: $\operatorname{Res}=\left\{\operatorname{Res}_{1}, \operatorname{Res}_{2}, \ldots \operatorname{Res}_{N}\right\}$, similar to the case of the reactive broker, where $N$ changes dynamically with user demand. Once requests have been scheduled, they cannot be cancelled from the scheduler. Thus, the broker guarantees execution of a request which has been accepted by the system, within the 
specified deadline. The broker is run in two separate threads as shown in Figure 5.2. RH is running in thread 1, listening for incoming requests from the User module. Thread 2 performs the auto-scaling operations.

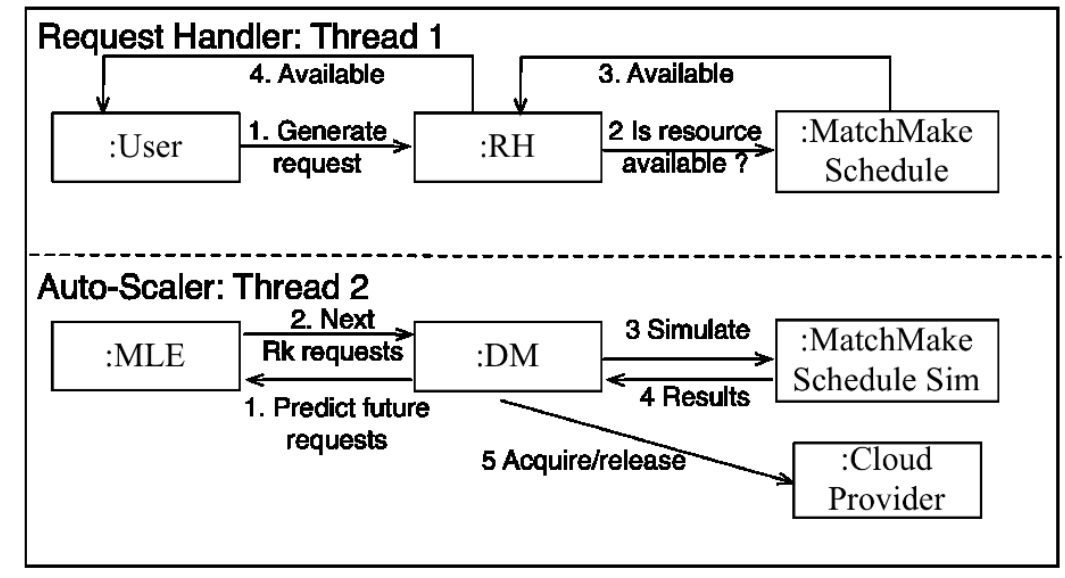

Figure 5.2: Proactive Broker: Collaboration Diagram

\subsubsection{Machine Learning Engine}

Figure 5.2 also shows the collaboration among the different components in the autoscaler (thread 2). At the beginning of each prediction operation, the next $k$ requests, denoted by $\mathrm{R}_{\mathrm{k}}$, are sent from MLE to DM. MLE predicts all the five characteristics, AT, EST, ST, $\mathrm{DL}$ and $\mathrm{T}$ for all $k$ requests, as described earlier in Section 3.1.2. A time-series analysis [58] has been used by MLE. Such an analysis is a natural fit because the available training data occurs in increasing order of time sequence. The training data is a sequence of tuples, each of which is characterized by a request arrival time, an earliest start time, an execution time, a deadline and a request type. We have experimented with two machine learning algorithms which use time series analysis: Linear Regression (LR) and Support Vector Regression (SVR). The machine learning algorithms predict the characteristics of future requests based on historical data.

After receiving the response for the characteristics of the $k$ predicted requests from 
MLE, DM invokes a simulation of the MMS to simulate the resource management operations for these predicted future requests using MMS. This is done by using the module MatchMakeSchedule Sim in Figure 5.2. Based on the output of MMS Sim, DM decides whether to acquire new resources or extend the stop time for existing resources.

Once DM decides to acquire a resource, it must procure the resource from a public cloud provider using DRPM. Additionally, each new resource acquired by the system has the following characteristics:

- $\quad$ Start time for the $i^{\text {th }}$ resource (start $\left.)\right)-$

$$
\operatorname{start}_{i}=E S T_{j}-T \text { minutes }
$$

- Here, $E S T_{j}$ is the EST of the $j^{\text {th }}$ request expected to execute earliest on the $i^{\text {th }}$ resource.

- [Note that EST was described in Section 3.1.2 and $T$ was described in Section 4.1]

- Stop time for the $i^{\text {th }}\left(\right.$ stop $\left._{i}\right)-$

$$
\text { stop }_{i}=\text { start }_{i}+\left\lceil\frac{\text { numSecond }_{i}^{p}}{3600}\right\rceil
$$

○ where numSeconds ${ }^{p}$ is the difference in seconds between the expected completion time of the last predicted request on resource $i$ and start $_{i}$. Since a typical public cloud provider charges by the hour, the resource is held until the end of this "paid hour" period. The superscript ' $p$ ' is to denote that the numSeconds is the predicted value for which the resource will be utilized.

The scale-up operation of the proactive auto-scaler is described in Figure 5.3 through a sequence diagram. Each existing resource is represented by a variable in the simulator, 
which enables the simulator to store the pending requests in each resource, and each of the $k$ predicted requests is processed by a simulated version of MMS. If the predicted request can be scheduled on an existing resource, no changes are made to the resource list and a boolean value $b$ is returned as true by MMS. However, if a new resource $\left(\operatorname{Res}_{j}\right)$ is needed to accommodate the request, DM suggests Res $\mathrm{j}_{\mathrm{j}}$ may be acquired. At the end of the simulation of $k$ requests, each new resource Resi, suggested by DM, serves $q$ requests $\left\{s t^{i}\right.$, $\left.s t^{i}, \ldots . s t_{q}^{i}\right\}$, where $s t_{q}^{i}$ is the amount of time in seconds that the $i^{t h}$ resource spends servicing the $q^{\text {th }}$ request. At the end of this simulation, DM decides to acquire actual resources using the scale up operation based on a profit calculating function given in Equation (10). This is shown in the loop which cycles through all predicted resources and acquires those resources that are profitable.

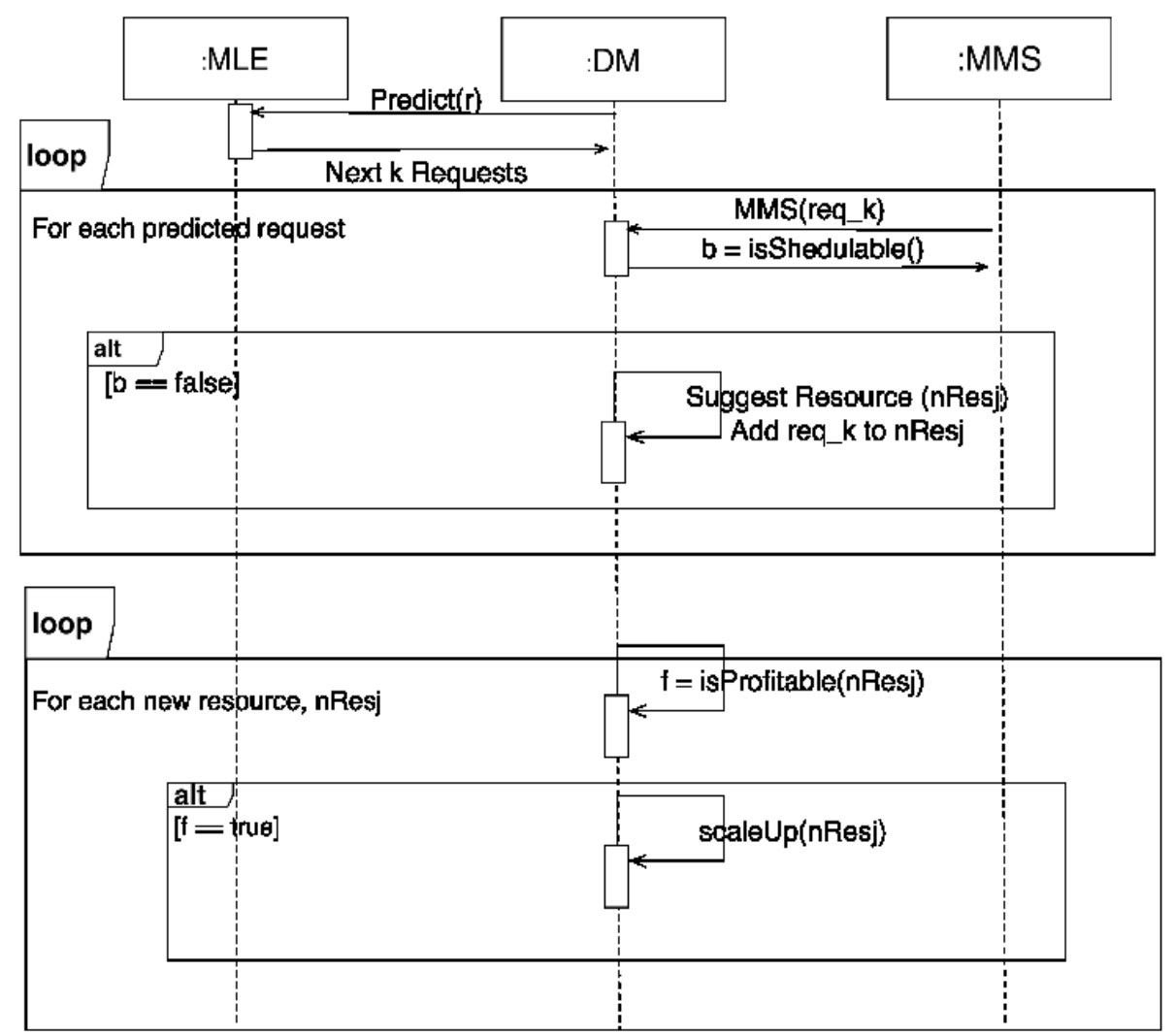

Figure 5.3: Proactive Broker: Sequence Diagram 


\subsubsection{Auto-scaling Rules}

As mentioned earlier in Section 3.2, $c \_p u b$ and $c \_p v t$ are the broker cost and user cost respectively. The following factors determine the predicted profit for the broker:

- Predicted Broker Cost $\left(\boldsymbol{B C}^{p_{i}}\right)$ - The predicted cost for running the $i^{\text {th }}$ resource is given by: $\left[\left(\right.\right.$ stop $_{i}-$ start $\left.\left._{i}\right)\right] \times c \_$pub

- Predicted service time $\left(s_{i}\right)$ - The predicted total service time for $q$ requests on the $i^{\text {th }}$ resource is given by: $\sum_{j=1}^{q} s_{j}^{i}$ in seconds.

The predicted broker cost is computed by considering the predicted start time $\left(\operatorname{start}_{m}{ }_{m}\right)$ and predicted end time $\left(\right.$ stopt $\left.{ }_{m}\right)$. Since the resource is already running, the predicted start time is the same as stop time of the resource $\left(\right.$ stop $\left._{m}\right)$.

- Predicted Total User Cost $\left(\boldsymbol{U C}^{p}{ }_{i}\right)$ - The predicted cost for the users to execute requests on the $i^{\text {th }}$ resource is given by: $\left[s_{i}\right] \times c \_p v t$

- Predicted Broker Profit $\left(\boldsymbol{B P}^{p_{i}}\right)$-The predicted profit earned by servicing user requests on the $i^{\text {th }}$ resource is given by:

$$
B P^{p}{ }_{i}=\left(U C^{p}{ }_{i}-B C^{p}{ }_{i}\right)
$$

Note that the superscript $p$ denotes predicted values. The actual values of these parameters are denoted by superscript $a . B P$ may be calculated for any time period and is referred to as $B P^{a}$ and $B P^{p}$ signifying the actual and predicted values respectively. The reason for having two values for broker profit is due to the proposed system having a prediction stage which auto-scales resources based on predicted profit from future workload. Hence, this predicted profit is associated with a superscript ' $p$ ' whereas the actual profit accrued by the broker during its operation is associated as the profit with the superscript ' $a$ '. The total cost saving for all requests sent by SCE is computed as the sum of 
savings achieved with all the requests that were processed by the system. The cost saving for a single request, with a service time of $c$ seconds is the difference between the cost that would be incurred if the resource were procured directly from the public cloud provider and that incurred when it is acquired from IE.

If $B P^{p}{ }_{i}$ is less than $0, \mathrm{DM}$ must check with the GoS module to ensure that the GoS criterion remains satisfied. The GoS criterion that was introduced in Section 3.1.1 is based on the blocking ratio. An explanation for $B$ may be found in Section 4.1. $B^{p}$ is continuously monitored on the system. Irrespective of whether a profit will accrue, a resource is acquired if:

$$
B^{p}>B_{\text {spec }}
$$

Similar to Section 4.1, the desired value of $B^{p}$ is maintained by the broker and is stored in the GoS component. Note that, $B^{p}$ is the predicted value of $B$, since the value is computed by accounting for requests which are predicted by MLE.

The predicted broker profit is used in the following way by DRPM that handles the task of communicating with the public cloud provider and acquiring or releasing resources. Acquiring and releasing a resource is based on these two rules:

- Rule I: When,

$$
B P^{p}{ }_{i}>0
$$

○ acquire $i^{\text {th }}$ resource

- Rule II: When,

$$
\text { stop }_{i}=\text { current time }
$$

$\circ$ release $i^{\text {th }}$ resource

- Rule III: If $\boldsymbol{B P}^{\boldsymbol{p}}{ }_{i}<\mathbf{0}$ and rejecting the request would violate the GoS 
criterion, acquire $j^{\text {th }}$ resource

Rule I states that the $i^{\text {th }}$ resource is acquired if a profit is predicted to be generated for the broker. Rule II specifies the release of the $i^{\text {th }}$ resource at its stop time. Rule III states that even if the calculated value of $B P_{i} \leq 0$, DM needs to ensure that the GoS criterion remains satisfied.

A limitation of the proactive auto-scaling technique is that it requires a training period to function properly. An alternate system may be proposed that functions in a reactive mode (System I-R), explained in Chapter 4, during the training and switches to the proactive system (System I-P) at the end of the training period.

\subsection{The Proactive Auto-Scaling Algorithm}

The proactive auto-scaling algorithm presented in Table 5.1 is described next. The algorithm determines whether new resources need to be acquired or not, based on predicted workload. The stop times are set in two cases, when a new resource is started or when an existing resource is determined to be profitable in the future and the stop time is extended. Acquisition of resources suggested by MMS follow Rule I. In the algorithm, variables used in computation are initialized in line 1. Next, DM invokes MLE to predict the next set of $k$ requests (line 2a). DM also creates a temporary variable containing information about the current system state the current resources and makes them available for simulation by MMS (line 2b). System state is characterized by the list of resources acquired by the system and the list of requests for each resource. Lines $3-13$ show the first loop, right after the $k$ requests are predicted, in the sequence diagram given in Figure 5.3. DM sends each request to MMS which decides whether the current set of resources can meet the request deadline. If not, a new resource is suggested to be acquired. Lines $14-21$ show the second loop in 
the sequence diagram given in Figure 5.3.

Table 5.1 Proactive Auto-Scaling Algorithm

1. Initialization: $\mathrm{nRes} \leftarrow\{\}$, count $\leftarrow 0$

2a. Predict the next k requests using MLE: $\left\{\operatorname{Req}_{1}, \operatorname{Req}_{2}, \ldots \operatorname{Req}_{\mathrm{k}}\right\}$

2b. Acquire the system state with $N$ resources $\left\{\operatorname{Res}_{1}, . ., \operatorname{Res}_{N}\right\}$

3. for each request $\mathrm{Req}_{\mathrm{i}}$, from $\mathrm{i}=1$ to $\mathrm{k}$ do

4. for each resource $R e s$, from $j=1$ to $N+$ count do

5. if $R_{e q}$ can be scheduled on $\operatorname{Res}_{j}$

6. Schedule Req

7. if $\operatorname{Res}_{\mathrm{j}}$. StopTime $<\mathrm{Req}_{\mathrm{i}}$.endTime

8. $\quad$ Res j.stopTime $_{\text {. }} \leftarrow \mathrm{Req}_{\mathrm{i}}$.endTime

9. break;

10. end for

11. if Req $i$ not scheduled

12a. Increment count by 1 .

12b. Schedule Req $\mathrm{i}_{\mathrm{i}}$ on $\mathrm{Res}_{\mathrm{N}+\text { count; }}$;

12c. $\quad n R e s \leftarrow \operatorname{ReS}_{\mathrm{N}+\text { count }}$.

13. end for

14. for all $n \operatorname{Res}_{i}$, from $i=1$ to count, do

15. $\mathrm{UC}_{\mathrm{i}}^{\mathrm{p}} \leftarrow\left[\mathrm{s}_{\mathrm{i}}\right] \times\left[\mathrm{c} \_\mathrm{pvt}\right]$

16. $\mathrm{start}_{\mathrm{i}} \leftarrow\left(\right.$ EST of earliest Req on $\left.\mathrm{nRes}_{\mathrm{i}}\right)-\mathrm{T}$

17. stop $_{\mathrm{i}} \leftarrow \operatorname{start}_{i}+\left\lceil\frac{\text { numSeconds }_{i}^{p}}{3600}\right\rceil$

18. $\mathrm{BC}_{\mathrm{i}} \leftarrow\left[\left(\right.\right.$ stop $\left.\left._{\mathrm{i}}-\mathrm{start}_{\mathrm{i}}\right)\right] \times\left[\mathrm{c} \_\right.$pub $]$

19. if $\left(\mathrm{UC}^{\mathrm{p}} \mathrm{i}-\mathrm{BC}_{\mathrm{i}}^{\mathrm{p}}\right)>0$

20. $\quad$ acquire $\left(\right.$ start $\left._{\mathrm{i}}, \mathrm{stop}_{\mathrm{i}}\right)$

21. end for

22. for all $\operatorname{Res}_{\mathrm{m}}$, from $\mathrm{m}=1$ to $\mathrm{N}$, do

23. $\mathrm{UC}_{\mathrm{m}}^{\mathrm{p}} \leftarrow\left[\mathrm{s}_{\mathrm{m}}\right] \times\left[\mathrm{c} \_\mathrm{pvt}\right]$

24. $\operatorname{start}_{\mathrm{m}} \leftarrow$ stop $_{\mathrm{m}}$

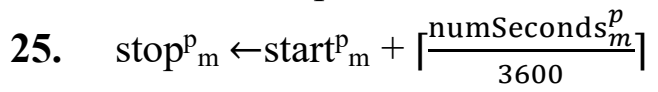

26. $B C^{\mathrm{p}}{ }_{\mathrm{m}} \leftarrow\left[\left(\right.\right.$ stop $\left.\left._{\mathrm{m}}^{\mathrm{p}}-\operatorname{start}_{\mathrm{m}}^{\mathrm{p}}\right)\right] \times\left[\mathrm{c} \_\right.$pub $]$

27. if $\left(\mathrm{UC}^{\mathrm{p}} \mathrm{m}-\mathrm{BC}^{\mathrm{p}}{ }_{\mathrm{m}}\right)>0$

28. $\quad$ stop $_{\mathrm{m}} \leftarrow$ stop $^{\mathrm{p}}{ }_{\mathrm{m}}$

29. end for

Profit calculation is performed by comparing $\mathrm{UC}^{p_{i}}$ for using the $i^{\text {th }}$ resource with $B C^{p}{ }_{i}$ for that resource. Only if there is a profit (using Rule I) a new resource is acquired (line 20) by setting a start and a stop time for the resource. Next, the algorithm loops through 
the existing resources to determine whether an extension in the stop time of a resource is profitable (lines $22-29$ ). If there are further requests pending on the resource, which make the resource profitable for further use (lines $27-28$ ), the stop time is extended. A request is rejected if there are no resources available to service the request.

\subsection{Experimental Setup}

This section describes the experimental setup used for analyzing the proactive autoscaling technique. Section 5.3.1 describes the programming language and framework used in the implementation of the simulator and the prototype. Section 5.3.2 describes the details about the implementation and challenges faced while deploying the broker on a real cloud environment.

\subsubsection{Programming Language/Framework}

The experimental platform is similar to the one described for the reactive system described in Section 4.3.1. One additional external project known as WEKA [59] has been used in this project.

- WEKA is a machine learning software used during the prediction operations performed by MLE.

\subsubsection{Implementation}

The implementation for the prototype is similar to the one described in Section 4.3.2.1 while the implementation for the simulator is similar to the simulator presented in Section 4.3.2.2 for the reactive technique. The difference is in the auto-scaler for the proactive and reactive techniques. While the proactive framework employs MLE to predict requests using the WEKA library and then acquires resources based on predicted broker profit, the reactive framework auto-scales based on profit for the broker on request arrival. 


\subsection{Experimental Parameters}

This section focuses on describing parameter values which are expected to affect the performance of the system as described in Section 5.5. Each experiment is performed by changing one parameter at a time while holding the other parameters at their default values (see Table 5.2). The default values are shown in boldface. Column 2 specifies the set of all values used for the respective parameter.

\subsubsection{Workload Parameters}

The workload parameters for the proactive system are identical to the parameters defined by the reactive system in Section 4.4.1.

\subsubsection{System Parameters}

The default public cloud provider cost is assumed as 2 cents for one hour of using a micro instance, the same used in Section 4.4.2. The same user cost described in Table 4.3 for the reactive auto-scaling technique is used. Table 5.2 describes two additional system parameters used by MLE for prediction.

- Linear regression (LR) is selected as the default machine learning algorithm (MLA) when running the experiments to analyze the performance of the proactive autoscaling system. To compare the system performance when using other machine learning algorithms, one experiment is performed which compares the broker profit accrued while using the Support Vector Regression (SVR) machine learning algorithm with that of the Linear Regression (LR) machine learning algorithm (please refer to Figure 5.12).

- The training data for these experiments were gathered for $40 \%$ of the total requests to assess the workload characteristics. During this time, the auto- 
scaling was not performed. The features predicted by MLE were the request characteristics defined in Section 3.1.2.

- The number of requests that arrive between two successive predictions for requests $(P)$ is varied during the experiments. This is the number of requests which must arrive at the system to trigger another prediction operation. The entire history of the request arrival patterns is used for this prediction. The default value of 10 used for $\mathrm{P}$ is small enough to account for any changes that may occur in the workload demand without running the machine learning algorithm too often.

Table 5.2: Summary of System Parameters for Proactive System

\begin{tabular}{|c|c|}
\hline Machine Learning Algorithm: $M L A$ & Linear Regression, Support Vector \\
& Regression \\
\hline Number of requests between Predictions: $P$ & $\mathbf{1 0}, 20,30,40,50,100$ \\
\hline
\end{tabular}

\subsubsection{Performance Metrics}

The performance metrics are the same as described in Section 4.4.3.

\subsubsection{System Overheads}

As indicated in Section 4.4.4 the average overhead of an operation is characterized as the average time spent by all the requests during an experiment on performing the given operation. The average overheads due to internal and external communication that were introduced in Section 4.4.4 are used in the context of the proactive system. The chapter introduces a new overhead caused by executing the machine learning algorithm (MLA).

Average Overhead due to $M L A$ : corresponds to the delay caused the Prediction Engine. It is defined as the difference between the time at which the MLE operation ceases 
and the time at which MLE operation starts. The overhead is aggregated over all predictions made during one experiment. The average overhead is calculated by aggregating all the delays caused by MLE during an experiment and dividing the sum by the total number of such delays in the experiment.

\subsection{Results}

This section presents the results obtained by the simulation framework. In addition to the simulator, the proof of concept prototypes for System I-P, System II and System III were run on a system using Amazon EC2 as the public cloud. The simulator and prototype are similar to those described in Section 4.3.2.1 and Section 4.3.2.2 respectively for the reactive system. However, the broker used in the proactive system is different from the broker used in the reactive system. This section presents the results obtained by framework using a prototype implementation using Amazon EC2 resources.

\subsubsection{Comparison between the Simulator and the Prototype}

A comparison of the measurement results with those of a simulated system showed close agreement. The system used a discrete event-based simulator that simulated the acquisition and release of resources using the same workload conditions. In most cases of $f$, a difference of $3 \%$ to $5 \%$ between the simulator and the prototype was observed with a largest difference of $6 \%$. A reason for this difference may be attributed to the time required in the prototype to send and receive requests using the network, which is not present in the simulator. However, the difference is small because the delay in communication is only a few hundred milliseconds and does not significantly affect the broker profit. The better performance of the simulator may be attributed to the assumption of zero communication latencies whereas the communication between the broker and the virtual resources as well 
as between SCE and the broker in the prototype are subject to non-zero communication latencies with network latencies due to Amazon's internal data center network and external networks having a small impact on broker performance for the prototype. Figure 5.4 presents a comparison between the broker's profits determined through measurements on the prototype system with that observed on a simulated system. The greatest difference between the two systems is $6 \%$ at $f=0$. At lower values of $f$, the difference between the $B P^{a}$ values determined in both systems is much smaller: for example, a difference of $3 \%$ is observed at $f=0.6$.

Since the difference in the results for the simulator and the prototype is small, the remaining experiments in this chapter have been run using the simulator, thereby saving costs associated with acquiring resources on a public cloud as well as saving time to run the experiments in the cloud environment. Note that the system overheads reported in Section 5.5.7 are based on measurements made on the system prototype. Additionally, a close match between the prototype and the simulator results was found for System II as well, and hence only the simulator results for System II have been presented in this chapter.

\subsubsection{Impact of Arrival Rate}

A performance analysis of a system subjected to a single arrival rate is performed to investigate the impact of $\lambda$ on profit. Figure 5.5, presents the effect of $\lambda$ on the profit generated on System I-P and System II. These systems have been introduced in Section 3.3. $B P^{a}$ in both systems seems to be directly proportional to the arrival rate. This is because at higher arrival rates, the system receives a higher number of requests per unit time, thus increasing the potential for earning a higher profit/hour by accepting a higher number of requests. System I-P earns in between 2.5 to 4 times the profit earned by System II. 


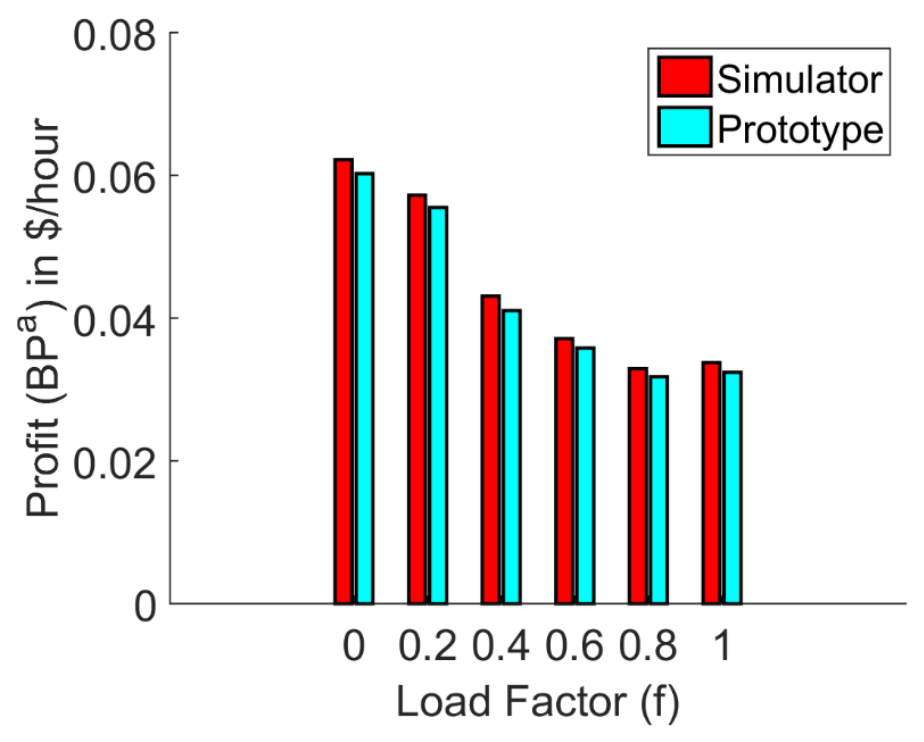

Figure 5.4: Comparison of the Simulator and Prototype for the Proactive Auto-scaler

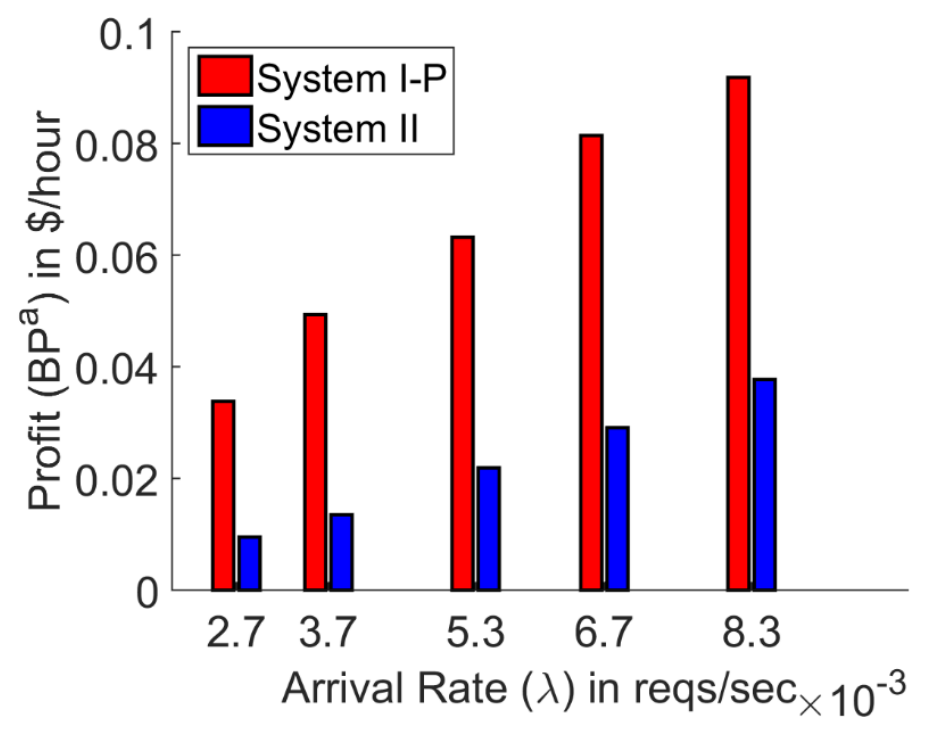

Figure 5.5: Effect of $\lambda$ on Broker Profit for the Proactive System

\subsubsection{Impact of Load Factor}

This sub-section describes the impact of $f$ on the various systems described in Section 3.3. In Figure 5.6, different values of $f$, discussed in Table 4.2, are used to compare the profit earned by the broker using Systems I and II. In all cases, System I-P performs better than System II, earning as high as 3.5 times the profit earned in System II. For any given 
system, the profit is inversely proportional to $f$ : as $f$ increases, profit decreases. For example, when $f$ is 0 , the profit/hour is the highest. Note that at $f=0$, the system is subjected to only the higher arrival rate. As $f$ increases, the proportion of requests with lower arrival rates decreases. At $f=1$ the system is subjected to only the lower arrival rate and the $B P^{a}$ earned are lower.

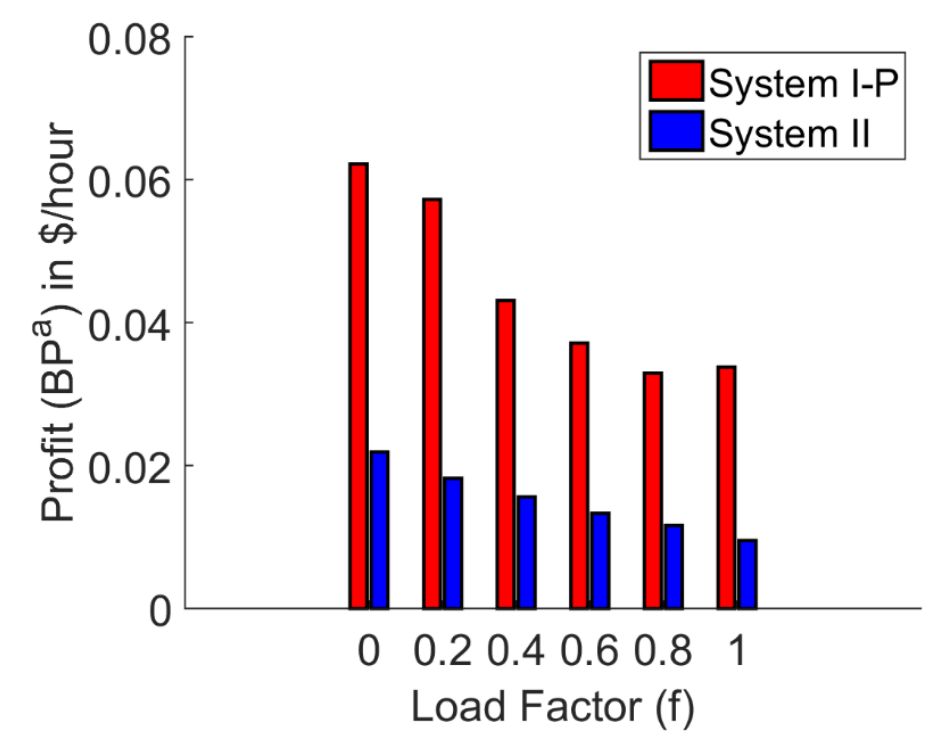

Figure 5.6: Effect of $f$ on Broker Profit for the Proactive System

Figure 5.7 presents a comparison of all the three systems. As shown in the figure, System I-P leads to a lower $U C^{a}$ in comparison to the other two systems. All requests are considered to be arriving from a group of users that belong to the client enterprise. Hence, an increase in arrival rate, the broker received more requests per unit time. This enables the broker to schedule more requests per unit time, thereby increasing broker profit by making the collective users pay a higher amount per unit time, increasing $U C^{a}$. Consequently, as seen in Figure 5.7, a lower $f$ results in a higher $U C^{a}$ thus enabling the broker to earn a higher $B P^{a}$. 


\subsubsection{Impact of Laxity Factor}

In Figure 5.8, Systems I and II are subjected to different values of $L f$. A $L f$ of 0 implies that there is has no additional slack time for the requests. This renders the autoscaling technique ineffective because the system cannot schedule multiple requests without missing some of the deadlines and thus the broker must reject a portion of the requests to guarantee satisfying the deadline requirement for accepted requests. Consequently, the profit is below 0 if most requests are rejected, providing the broker a lower opportunity for accruing a profit. However, as the laxity in the system increases, $B P^{a}$ also increases. A 150 $\%$ improvement in $B P^{a}$ is observed at Lf of 5 in comparison to keeping the $L f$ at 2 . Overall, for a given value of $L f$ between 2 and 5, System I-P performs 3.5 to 4 times better than System II.

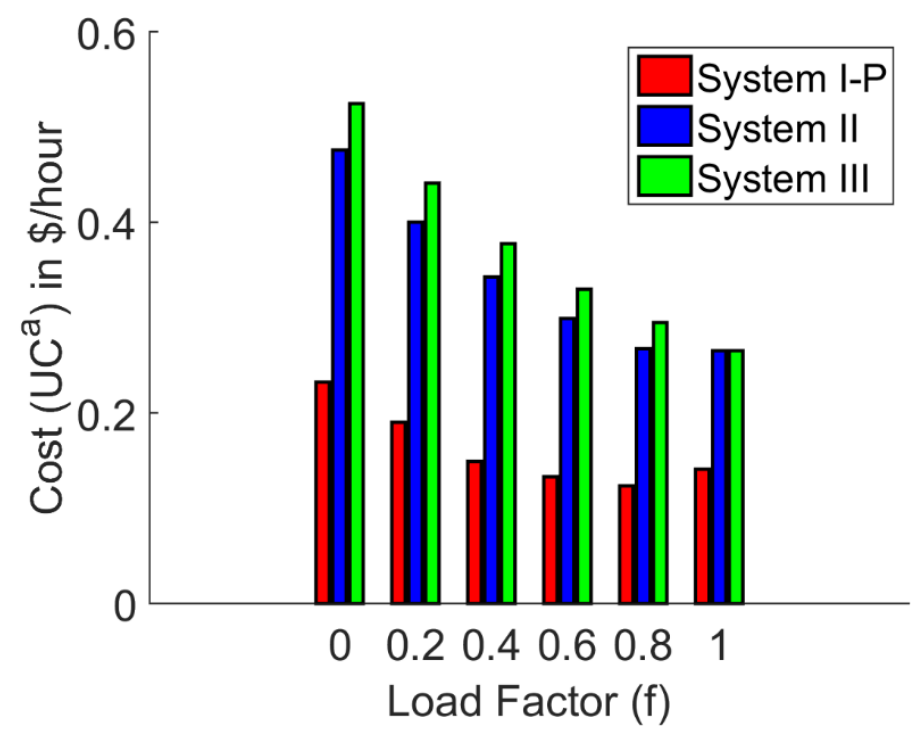

Figure 5.7: Effect of $f$ on User Cost for the Proactive System

\subsubsection{Impact of Service Time}

Figure 5.9 captures the impact of $S$ on the performance of System I-P and System II. The figure shows that the profit/hour increases with an increase in $S$. This is because the 
larger the $S$, the higher the utilization of the resources that leads to a higher revenue for the broker. For any given $S$, System I-P exhibits a significantly superior performance in comparison to System II. Note that for the default parameters used in this experiment, it would take at least 40 minutes of requests running on a resource per hour for the system to break even and avoid a loss. More details about why 40 minutes is the key time period between registering a profit or a loss may be found in Section 4.5.6.

\subsubsection{Impact of User Cost Rate}

In Figure 5.10, the effect of changing values of user cost rate, $c \_p v t$, on $B P^{a}$ is presented for different values of broker cost rate, $c \_p u b$. Varying the broker cost rate (c_pub) from to $\$ 0.02$ to $\$ 0.03$ per hour, the user is charged between $\$ 0.00000694$ and $\$ 0.0000139$ per second. Profit is observed to increase proportionally with an increase in the user cost. Since a higher value of $c \_p v t$ generates a higher charge to the user, the profit accrued by the broker also rises. When the value of $c \_p v t$ is equal to or less than c_pub, the algorithm does not incur any profit.

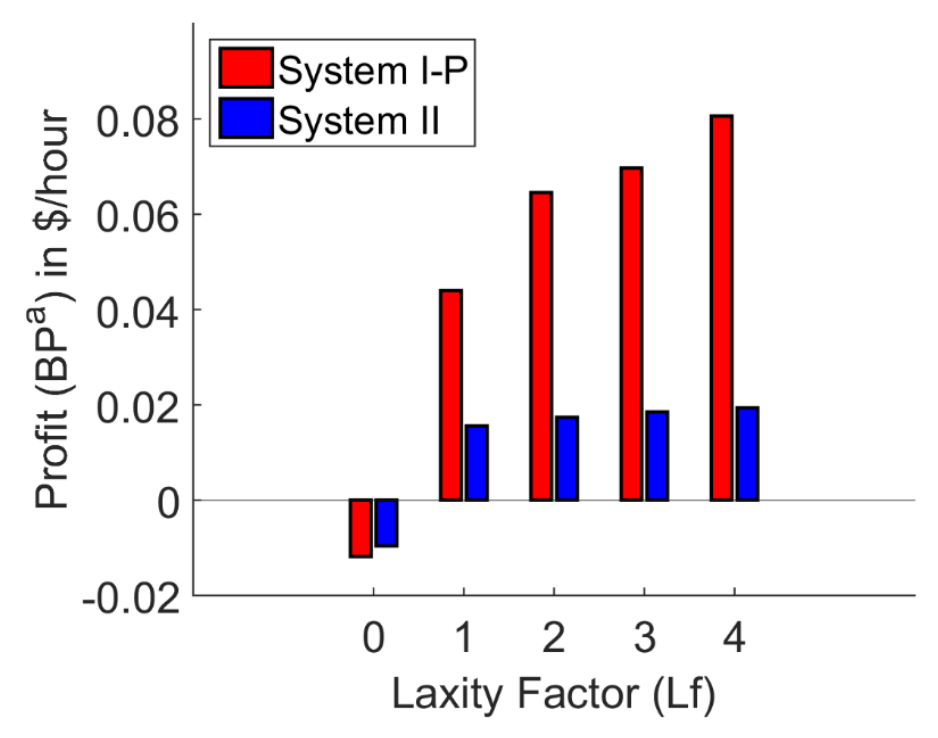

Figure 5.8: Effect of $L f$ on Broker Profit for the Proactive System 


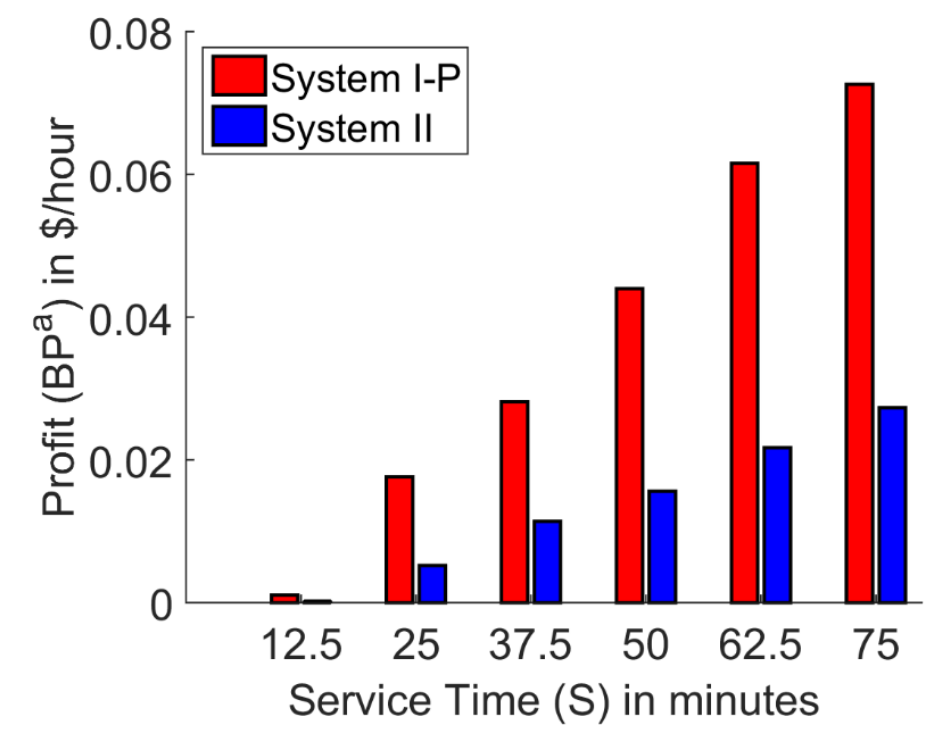

Figure 5.9: Effect of $S$ on Broker Profit for the Proactive System

\subsubsection{System Overheads}

An experiment was conducted with all the system and workload parameters held at their default values. The average overhead due to internal communication (discussed in Section 5.4.4) is measured to be 69 milli-seconds whereas the average delay due to external communication (also discussed in Section 5.4.4) is measured at 195 milli-seconds. The average overhead caused due to running the machine learning algorithm (defined in Section 5.4.4) is measured to be 6.2 seconds. The overheads are very small in comparison to the mean service time of a request, which is 50 minutes, or 3000 seconds.

\subsubsection{Other Results}

- Impact of Number of Requests between Predictions: Profit is observed to increase initially with an increase in the number of requests between predictions used by MLE. This is shown in Figure 5.11.

However, it reaches a peak at 50 and then decreases as the number of predictions is increased further. Small values of $\mathrm{P}$ do not allow MLE to properly 
learn the request behaviour. Also, when $\mathrm{P}$ is too large, stale information may get included in the prediction process, thus reducing the accuracy of predictions.

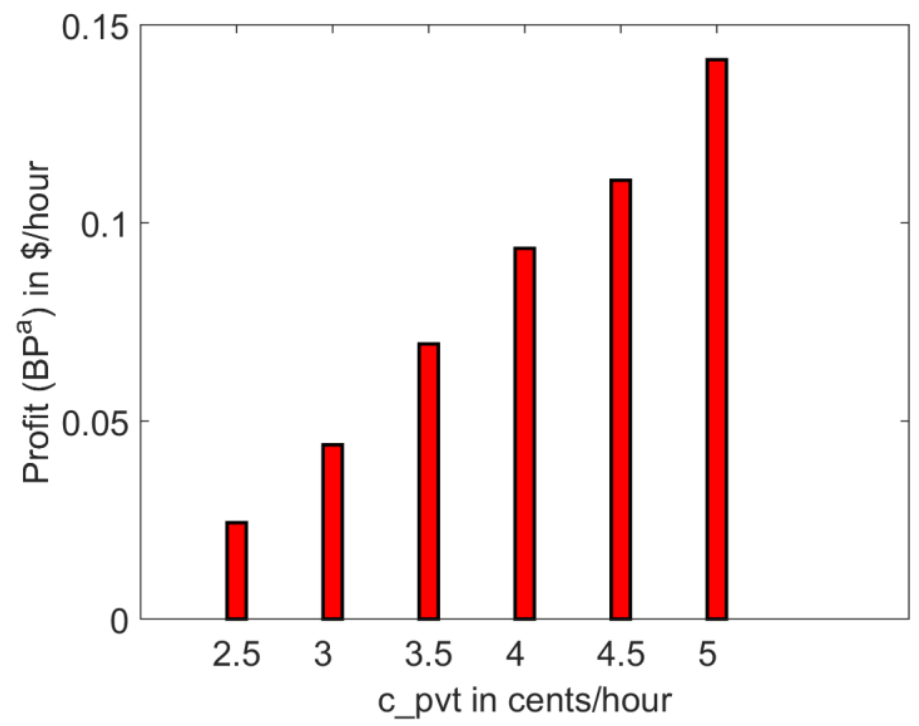

Figure 5.10 Effect of $c$ p pvt on Broker Profit for the Proactive System

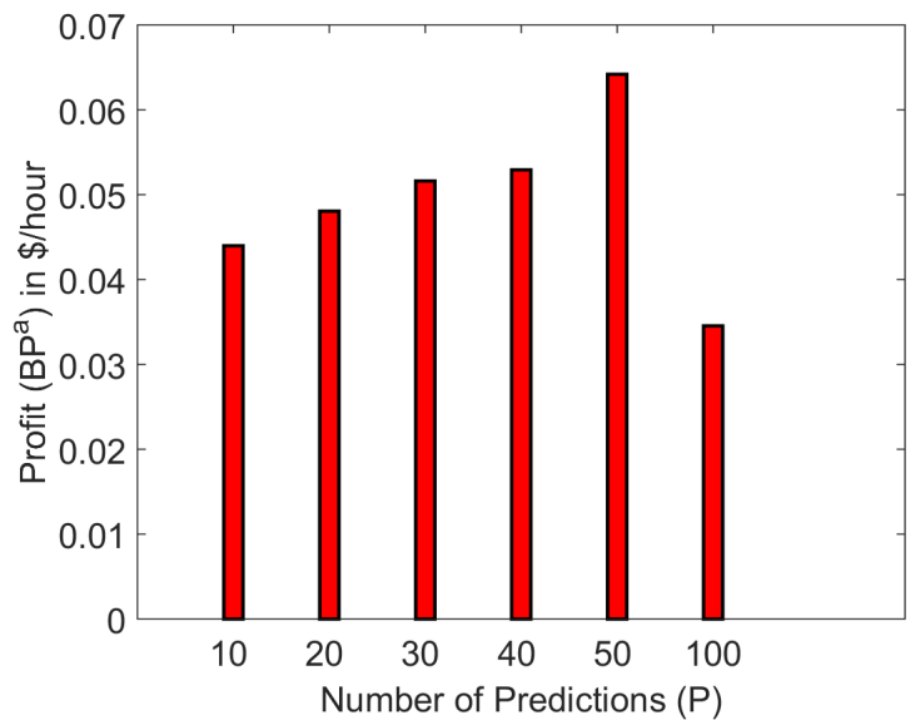

Figure 5.11: Effect of $P$ on Broker Profit for the Proactive System

- Impact of Machine Learning Algorithm: The improvement in $B P^{a}$ achieved by SVR over LR is marginal: $2 \%$ to $4 \%$ and is shown in Figure 5.12. The prediction error for the four AR request characteristics AT, EST, ST and DL observed at the default 
value of $f=0.4$ is presented in Table 5.3. For any given characteristic X, associated with a specific request arrival, the percentage error is computed as:

$$
\text { Error }=\left|\frac{\text { predicted } X-\operatorname{actual} X}{\text { predicted } X}\right| \times 100
$$

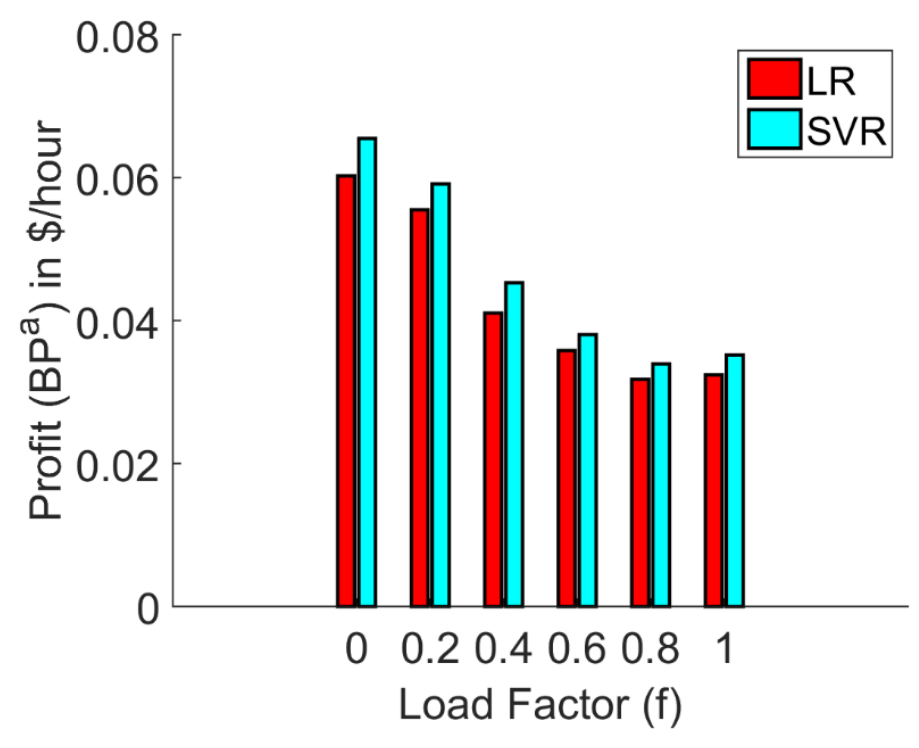

Figure 5.12: Effect of changing the $M L A$ on Broker Profit for the Proactive System

Table 5.3: Average Percentage Error in Prediction using LR and SVR

\begin{tabular}{|c|c|c|c|c|}
\hline Request Characteristic & AT & EST & DL & ST \\
\hline Linear Regression & $1.2 \%$ & $13 \%$ & $9.6 \%$ & $22 \%$ \\
\hline Support Vector Regression & $0.8 \%$ & $6 \%$ & $5.3 \%$ & $15 \%$ \\
\hline
\end{tabular}

Note that predicted $\mathrm{X}$ is noted when MLE generates a prediction and actual $\mathrm{X}$ is obtained after the request arrives on the system. The average error for $\mathrm{X}$ computed across all the requests generated in the experiment is reported in Table 5.3. A lower prediction error achieved with SVR leads to a marginally higher proportion of accepted requests. The proportion of accepted requests $(1-B)$ for LR and SVR for $f=0.4$ is 0.91 and 0.94 respectively. This leads to a slightly higher 
broker profit achieved with SVR in comparison to LR (see Figure 5.12).

\subsection{Observations}

The proactive auto-scaling technique introduced in the chapter combines a machine learning based predictive approach with a deadline driven algorithm for auto-scaling resources. The proposed framework is implemented as a prototype on a real cloud provider's system to demonstrate that a significant profit can be generated for the intermediary enterprise managing a virtual private cloud for a client enterprise while reducing the cost incurred by the users. The chapter also discusses the various overheads associated with the system and demonstrates that these overheads are small. A detailed performance analysis (broker profit and user cost) based on measurements made and a number of key observations achieved with a given set of system and workload parameters experimented with is presented:

- The experimental results demonstrate that using the proposed proactive broker can lead to a higher profit as compared to other non-proactive systems. For example, as shown in Figure 5.6, an improvement in $B P^{a}$ up to $250 \%$ of System I-P over System II has been observed. The broker profit is observed to depend on parameters, such as load factor, arrival rate as well as number of requests used in arriving at the predictions.

- A higher mean arrival rate enables the broker to earn a higher profit. As a result, lower the load factor, higher is the difference between the profit generated by the proactive system and that generated by a conventional system.

- The broker profit is directly proportional to the cost charged to the users of the single client enterprise and is thus observed to increase with increase in $S$. 
- The broker profit increases with the increase in $L f$.

- The user cost is observed to be directly proportional to the load factor while the broker profit is observed to be directly proportional to mean service time.

- Changing the machine-learning algorithm has a small impact on the broker profit with an algorithm such as SVR performing marginally better than linear regression. 


\section{Chapter 6 The Hybrid Auto-scaling Technique}

The hybrid auto-scaling technique described in this chapter combines the previous reactive and proactive auto-scaling techniques presented in Chapter 4 and Chapter 5 respectively. The motivation behind investigating a hybrid approach to auto-scaling is to deal with the limitations of the purely proactive and reactive methods. In a pure reactive auto-scaler, the scaling of resources takes time and the auto-scaler may miss opportunities to meet request deadlines. In a proactive auto-scaler, resources are available prior to request arrival. The required number of resources is predicted using a machine learning algorithm. However, errors in the prediction technique may cause requests to be dropped due to unavailability of resources when the requests actually arrive. The hybrid approach combines both approaches by proposing an auto-scaler that can scale both proactively and reactively, thereby striving to improve the system performance measured by broker profit and user cost in comparison to the pure proactive and pure reactive approaches.

The components of the framework that implements the hybrid auto-scaling algorithm is described in Section 6.1. The hybrid auto-scaling algorithm is described in Section 6.2. The experimental setup for performing the analysis of the auto-scaling technique is present in Section 0 while the workload and system parameters for the experiments are described in Section 6.4. Next, Section 6.5 presents the results obtained by experimenting with this auto-scaling framework. An analysis to find the profitability of the broker is made in 6.6. The key observations and insights resulting from the investigation of the hybrid auto-scaling technique are presented in Section 6.7.

\subsection{Broker Architecture}

The broker architecture is similar to that introduced in Section 3.1.1. The most important 
component in this broker is the hybrid auto-scaler. Similar to the reactive auto-scaler, this auto-scaler is run on every request arrival and it decides whether or not to acquire additional resources and whether or not the incoming request is to be accepted. Upon acceptance of a request, the broker guarantees that the request will be completed by the deadline included in the SLA. Another auto-scaler, similar to the proactive auto-scaler described in Chapter 5 runs periodically. after a predetermined number of request arrivals, and attempts to acquire resources proactively.

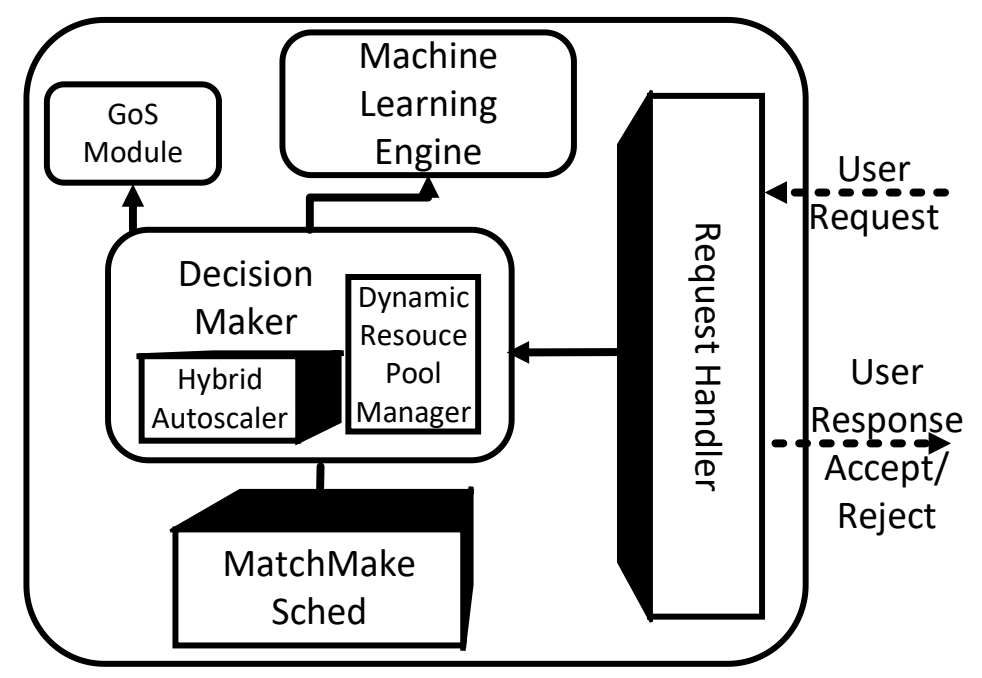

Figure 6.1: Hybrid Broker Architecture

The broker is responsible for the matchmaking and scheduling operations for an incoming request as well as auto-scaling a pool of resources. The following subsections describe the components of the broker system which is shown in Figure 6.1. The system comprises of five distinct components. The first component is the Request Handler (RH) that accepts requests from SCE. The primary responsibility of RH is to receive requests from users and forward the requests to MMS which decides whether to accept or reject the request. Operations of RH and MMS have been described in Section 3.1.3 and Section 3.1.4 respectively. The third component is Decision Maker (DM) which controls when 
resources are scaled. DM uses the fourth component known as Machine Learning Engine (MLE) to predict future requests based on past workloads. The final component is the GoS module, which stores the grade of service set by SCE, responsible for maintaining a QoS.

\subsubsection{Auto-scaling criteria}

Upon request arrival, the hybrid algorithm determines whether the incoming request is to be accepted (determined by MMS) and if accepted, whether additional resources need to be acquired to satisfy the request. Upon acceptance of an AR request, this request must be completed by a deadline included in the SLA. On request arrival, a resource is acquired if:

(i) No available resource satisfies the request SLA

$A N D$

The acquisition of the resource will lead to a profit

OR

(ii) Rejecting the request will lead to a $B$ that is higher than the specified GoS.

Note: A resource is released after the timer set on the resource expires and no further requests are scheduled on that resource. Further details on resource timer may be found in Section 4.1.

After every $k$ request arrivals, the broker also runs the proactive algorithm to calculate the appropriate number of resources needed in the future to satisfy the workload demands. The proactive auto-scaling approach used in the proposed technique is based on a time series analysis [60] using different machine learning algorithms. The machine learning engine (MLE), utilizes the functions of a software library called Weka [59]. The request characteristics described in Section 3.1.2 are predicted by MLE. The algorithm accomplishes the prediction by looking at past workload trends and predicts the workload 
in the future. The prediction is performed by extrapolating the appropriate number of resources required to satisfy the demand while maintaining the GoS as well as accruing a profit for the broker. A set of resources are acquired if:

(iii) No available resources satisfy one or more of the upcoming requests $A N D$

The upcoming predicted set of requests accrue a profit for the broker.

\section{OR}

(iv) Rejecting the upcoming predicted requests leads to a $B$ that is higher than the specified GoS.

The reactive and proactive auto-scaling techniques incorporated in the hybrid autoscaler are presented next.

\subsubsection{Reactive Auto-scaling}

Even though the incoming request is handled by $\mathrm{RH}$, the request handling process involves other modules of the broker. At any time, the broker controls a set of $N$ resources in the VPC: $\operatorname{Res}=\left\{\operatorname{Res}_{1}, \operatorname{Res}_{2}, \ldots \operatorname{Res}_{N}\right\}$, where $N$ changes dynamically with user demand. The broker is run in two separate threads and each thread runs independently of the other thread. The two threads decide on when auto-scaling operations are required without being influenced by the functions of the other thread. RH runs in Thread 1, listening for incoming requests from the User module and is activated on every request arrival. Thread 1 also runs a reactive auto-scaling component. This component decides to scale up and add an additional resource when the current resources controlled by the broker are not adequate to satisfy the request. To acquire a new resource, auto-scaling criteria (i) or (ii), defined in Section 6.1.1 are used.

Each thread can trigger a change in the number of resources and thus helps perform the 
auto-scaling operations (although they run independent of one another). Once requests have been scheduled, they cannot be cancelled from the scheduler. The decision to accept or reject the request is sent back to the user.

Thread 1 also uses MMS, DM as well as the GoS module. One of the components of DM is the Reactive Auto-scaler (RA). MMS, running inside Thread 1 determines whether the request can be scheduled on an existing resource such that its SLA is satisfied (see Section 3.1.4). If so, the request is accepted. If no existing resource can satisfy the SLA requirements of the request, criteria (i) and (ii) described in Section 6.1.1 are used to determine whether an additional resource is to be acquired so that the request can be accepted. If no additional resource can be acquired, the request is rejected. For calculating the broker profit, used by the criteria, equation (13) is used. It is similar to equation (1) used in Section 3.2. except that the equation is modified to accommodate the characteristics of a particular request $i$. For this $i^{\text {th }}$ request having a service time of $c_{i}$ seconds, RA checks it against the following condition:

$$
B P_{i}>0
$$

Where $B P_{i}$ is defined as:

$$
B P_{i}=\boldsymbol{G} \times\left(\boldsymbol{c}_{\boldsymbol{i}} \times \boldsymbol{c}_{-} \boldsymbol{p} \boldsymbol{v} \boldsymbol{t}\right)-\left(\left\lceil\frac{\boldsymbol{c}_{\boldsymbol{i}}}{\mathbf{3 6 0 0}}\right\rceil \times \boldsymbol{c}_{-} \boldsymbol{p} \boldsymbol{u} \boldsymbol{b}\right)
$$

As mentioned earlier in Section 3.2, c_pub and $c \_p v t$ are the broker cost and user cost that are incurred by the broker and SCE respectively. $G$ is a constant greater than 1. A more detailed discussion of $G$ is presented in Section 4.1. This equation for calculating the broker profit determines whether the cost of acquiring the resources from the public cloud by the broker is lower than the fees earned from the SCE. When the criterion is met and $B P_{i}$ is greater than 0 , and DM issues a command to DRPM, a new resource is acquired. However, 
the resource must be acquired only for the time interval required by the request. This is to ensure that the broker does not have idle resources wasting money for IE. Hence, to achieve this, each new resource acquired by the system has the following characteristics:

- Start time for the $j^{\text {th }}$ resource (start $\left.t_{j}\right)$ -

$\circ$ Start $_{j}=\left(E S T\right.$ of the request expected to execute earliest on the $j^{\text {th }}$ resource $)-$ $\boldsymbol{T}$

- Resources are not made available immediately after a request is submitted by a public cloud provider and the additional $T$ seconds is used to account for the time to start a resource by the public cloud provider. More details may be found in Section 4.1.

- Stop time for the $j^{\text {th }}\left(\right.$ stop $\left._{j}\right)$ -

$$
\bigcirc \quad \text { stop }_{j}=\text { start }_{j}+\left\lceil\frac{\text { numSeconds }}{3600}\right\rceil
$$

Here, numSeconds is the difference in seconds between the expected completion time of the last request on resource $j$ and $s t a r t j$. A ceiling function converts an integer to return the next highest integer value by rounding up value, if necessary. Since a typical public cloud provider charges by the hour, the resource is held until the end of this "paid hour" period. Each resource $j$ has an internal timer which is set to expire at the time stop $_{j}$. Hence, even though the resource must be switched off after the request finishes execution, any remaining time might be used by another request.

If $B P_{i}$ is less than $0, \mathrm{RA}$ must check with another component that makes use of Thread 1. This component is known as the GoS component. Even though the broker does not accrue a profit from the request, RA needs to check whether the GoS criterion is satisfied. RA consults with the GoS component that contains configuration details set by SCE. The 
GoS criterion used in this research is based on the blocking ratio. An explanation for $B$ may be found in Section 4.1. B is continuously monitored on the system. Irrespective of whether a profit will accrue, a resource is acquired if:

$$
B>B_{\text {spec }}
$$

Similar to Section 4.1, the desired value of $B$ is maintained by the broker and is stored in the GoS component.

Hence, if none of the available resources can satisfy the SLA for request i the broker uses the following rules to decide whether to acquire a resource from the public cloud.

- Rule I: When $B P_{i}>0$, acquire $j^{\text {th }}$ resource

- Rule II: When $B>B_{\text {spec }}$, acquire $j^{\text {th }}$ resource

To release a resource, this rule is followed:

- Rule III: When $\left(s t o p_{j}\right)=$ current time, release $j^{\text {th }}$ resource

Rule I states that the $j^{\text {th }}$ resource is acquired if a profit is generated for the broker. Rule II states that the request must be accepted if the system is failing to meet the specified grade of service guaranteed by IE. Rule III specifies the release of the $j^{\text {th }}$ resource occurs when the current system time is equal to the stop time of the resource.

\subsubsection{Proactive Auto-scaling}

Thread 2 is responsible for proactive auto-scaling and is periodically invoked. The hybrid auto-scaler running inside DM auto-scales proactively using two helper modules, GoS and MLE. When auto-scaling proactively, DM uses MLE to predict user demand. MLE uses a machine learning algorithm to predict future workload. Initially, MLE requires a training period to operate accurately. In this research, the training period is chosen as 
$10 \%$ of the total experiment run time. During this phase, no requests are accepted by the system. However, the system may be modified to accept requests and schedule them during this period in a reactive manner. Once the training phase is completed, the system can start normal operations. DM invokes the operation of MLE after every $k$ requests. MLE predicts the characteristics of the upcoming k requests, $\operatorname{Req}=\left\{\operatorname{Req}_{1}, \operatorname{Req}_{2}, \ldots, \operatorname{Re} q_{k}\right\}$.

In this chapter, the majority of experiments are conducted using Linear Regression (LR) [61] as the machine learning algorithm. Each request characteristic is predicted separately using a time series analysis. MLE allows other machine learning algorithms to work, in place of LR. In addition to LR, another machine learning algorithm known as Support Vector Regression (SVR) [62] has also been discussed in Section 6.5.7.2.

The operations of Thread 2 are discussed next. At the beginning of each prediction operation, DM requests for the next $\mathrm{k}$ requests, denoted by $R_{k}$, to arrive from MLE. All the five characteristics, AT, EST, ST, DL, that is predicted by MLE, have been described earlier in Section 3.1.2, for each of the k predicted requests. After getting a response, DM simulates the resource management operations for these predicted future requests using MMS. This invokes a mock scheduling and matchmaking operation. However, from the perspective of MMS, it works the same way and does not differentiate between a real request sent by SCE and the predicted request sent by DM.

Based on the output of MMS, DM decides whether to acquire new resources or change the stop time for existing resources in order to schedule the requests on the existing resource, similar to the purely proactive technique described in Section 5.1. After receiving the characteristics of the $\mathrm{k}$ predicted requests from MLE, DM invokes MMS and gets back a true or false response. If MMS can successfully schedule the request on one of the 
existing resources, it returns a true value and DM takes no further action (except adjusting the stop time of the resource, if required). However, if MMS is unable to schedule the predicted request, DM has to decide whether a resource will need to be procured in the future using the auto-scaling criteria (iii) and (iv) described in Section 6.1.1. It should be noted that even though the requests are only predicted, DM needs to ensure that it consults the GoS module to avoid a request rejection that can cause $B$ to violate the agreed threshold. The system avoids the situation of $B$ crossing the threshold set by $B_{\text {spec }}$ by deciding to accept the request when it arrives on the system.

Each new resource, acquired based on the predictions, has the same start and end time characteristics as described for the situation in which a new request arrival triggers a resource acquisition Hence, the following factors must be taken into consideration when determining the predicted profit for the broker, finally leading to a predicted profit function:

- Predicted Broker Cost $\left(\boldsymbol{B} \boldsymbol{C}_{i}^{p_{i}}\right)$ - The predicted cost for using the $i^{\text {th }}$ resource is given by: $\left[\left(\right.\right.$ stop $_{i}-$ start $\left.\left._{i}\right)\right] \times c_{\text {p pub. }}$. This should be kept as low as possible to maximize broker profit.

- Predicted service time $\left(\boldsymbol{s}_{i}\right)$ - The predicted total service time for $k$ requests on the $\mathrm{i}^{\text {th }}$ resource is given by: $\sum_{j=1}^{k} S T_{j}^{i}$ in seconds

- Predicted Total User Cost $\left(\boldsymbol{U C}^{p}{ }_{i}\right)$ - The predicted cost for the users to execute requests on the $i^{\text {th }}$ resource is given by: $\left[s_{i}\right] \times c \_p v t$

- Predicted Broker Profit $\left(\boldsymbol{B P}_{i}{ }_{i}\right)$-The predicted profit earned by servicing user requests on the $i^{\text {th }}$ resource is given by:

○ $B P^{p}{ }_{i}=\left(U C^{p_{i}-B C^{p}}{ }_{i}\right)$ 
If $B P^{p}{ }_{i}$ is greater than 0 , then the request is accepted. Note that the superscript $p$ denotes predicted values. The actual values of these parameters are denoted by superscript $a$ and used in Section 6.5. The predicted broker profit is used in the following way by DRPM that handles the task of communicating with the public cloud provider and acquiring or releasing resources as described in Section 3.1.5. Acquiring and releasing a resource is performed by using rules that are similar to the rules described in Section 6.1.1.

To acquire resources, these two rules are followed:

- Rule IV: When $B P^{p}>0$, acquire $j^{\text {th }}$ resource

- Rule V: When $B^{p}>B_{\text {spec }}$, acquire $j^{\text {th }}$ resource

To release resources, the following rule is followed:

- Rule VI: When $\left(s t o p_{j}\right)=$ current time, release $j^{\text {th }}$ resource

\subsection{Hybrid Algorithm}

The hybrid auto-scaling algorithm is divided into two sections. The first section describes the reactive component of the auto-scaling algorithm whereas the second describes the proactive component.

\subsubsection{Reactive Component}

The reactive auto-scaling algorithm given in Table 6.1 is described next. For each incoming request $R e q_{i}$, the algorithm is executed. Before a resource can be acquired, MMS must determine whether the existing set of resources can accommodate the request. This is shown in Lines $1-7$. DM sends each request to MMS which decides whether one of the resources in the current set of resources can be allocated to meet the request deadline. Acquisition of resources is based on the RA algorithm that follows Rule I and Rule II, 
specified in Section 6.1.2.

Table 6.1 Hybrid Auto-Scaling Algorithm: Part 1

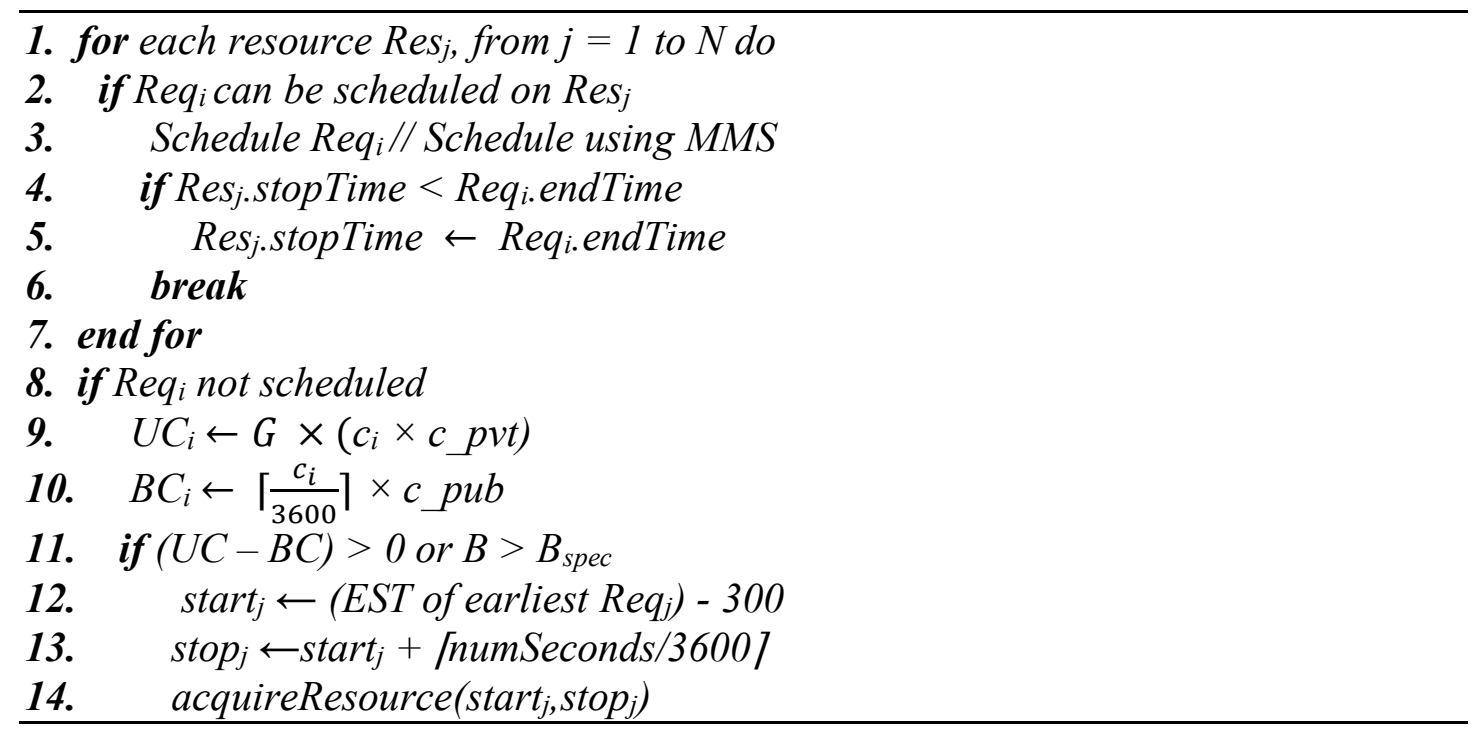

If the request cannot be accommodated by the existing set of resources, the reactive auto-scaling algorithm must be executed to determine whether a profit may accrue from the request after acquiring a new resource from the public cloud provider and scheduling the new request on that resource. An exception to this case is when the value of $B$ goes above $B_{\text {spec. }}$ This follows from Rule II that is associated with a scaling up operation. In this case, the resource is acquired, irrespective of whether a profit is generated by scheduling the new request on a newly acquired resource in order to meet the GoS. The process of resource acquisition is shown in lines $8-14$. The start and stop time of the resource to be acquired are set in line 12 and line 13 respectively. Line 14 performs the action of acquiring the new resource by communicating the request with DRPM. Resources are released when the current time reaches the stop time computed in line 13 and stored in the resource's respective timers.

The algorithm describes only the scale up operation. Resources are scaled down 
automatically when the current time becomes greater than the stop time of the resource as described in Rule III. The resource stop time is set in two cases, when a new resource is started or when the stop time is extended for an existing resource when executing a new request on the resource is deemed profitable.

\subsubsection{Proactive Component}

The two parts of the reactive auto-scaling algorithm (Table 6.2 and Table 6.3) are described next. The algorithm in Table 6.2 determines whether new resources need to be acquired or not, based on predicted workload. The stop time is set in two cases, when a new resource is started or when an existing resource is determined to be profitable in the future by executing a newly arrived request and the stop time is extended. The first part of the algorithm described in Table 6.2 presents the scenario of re-using existing resources without acquiring any new resources. The second part of the algorithm is presented in Table 6.3 and is only required if the current set of resources controlled by the broker cannot serve the upcoming requests.

Acquisition of resources suggested by MMS follow Rule IV and Rule V. DM invokes MLE to predict the next set of $\mathrm{k}$ requests before starting this algorithm. The system state is characterized by the list of resources acquired or currently controlled by the broker and the list of requests for each resource. Lines $1-13$ describes how DM sends each request to MMS which decides whether the current set of resources may meet the request deadline. If not, a new resource may be acquired if Rule IV and Rule V are satisfied. Each predicted request is sent to MMS to predict whether the request may be scheduled on an already existing resource. If the request cannot be scheduled using the existing set of resources, DM uses the algorithm (shown in Table 6.3) to determine whether acquiring a resource is 
profitable. Profit calculation is performed by comparing $U C^{p}$ for using the $i^{\text {th }}$ resource with $B C^{p}{ }_{i}$ for that resource. Only if there is a profit (using Rule IV) a new resource is acquired (line 5) by setting a start and a stop time for the resource. Resources are scaled down automatically when the current time becomes greater than the stop time as described in Rule VI.

Table 6.2 Hybrid Auto-Scaling Algorithm: Part 2a

1. for each pred. request Req, from $i=1$ to $k$ do

2. for each resource Resj, from $j=1$ to $N$ do

3. if Req $q_{i}$ can be scheduled on Res

4. $\quad$ Schedule Reqi

5. $\quad$ if Resj.stopTime $<$ Reqi.endTime

6. Res.stopTime $\leftarrow$ Reqi.endTime

7. break

8. end for

9. if Reqi not scheduled

10. Schedule Reqi on Res $N+$ count;

11. Increment count by 1.

12. $n \operatorname{Res} \leftarrow \operatorname{Res} N+$ count.

13. end for

14. Call selectResource ()

Table 6.3 Hybrid Auto-Scaling Algorithm: Part 2b (SelectResource)

1. for all $n$ Res, from $i=1$ to count, do

2. $U C_{p}{ }^{i} \leftarrow\left[s_{i}\right] \times\left[c \_p v t\right]$

3. $B C_{p}{ }^{i} \leftarrow\left[\left(\right.\right.$ stop $_{i}-$ start $\left.\left._{i}\right)\right] \times\left[c \_\right.$pub $]$

4. updateBO

5. if $B C_{p}{ }^{i}>0$ or $B^{p}>B_{\text {spec }}$

6. acquire(start stop $_{i}$ )

7. end for

\subsection{Experimental Setup}

Experiments are performed using both the simulator and the proof of concept prototype developed for the hybrid system. The simulator and prototype developed for the 
reactive component are similar to those for the pure reactive technique described in Section 4.3.2. The simulator and prototype developed for the proactive component are similar to those for the pure proactive technique described in Section 5.3.2. Additional code is included in the hybrid system to make the proactive and reactive components run simultaneously.

\subsection{Experimental Parameters}

This section focuses on how changing parameter values affects the performance of the system. The workload parameters for the hybrid system are identical to the parameters defined for the pure reactive system in Section 4.4.1. Similarly, the system parameters are identical to the ones described in Table 4.3 and Table 5.2 for the pure reactive and pure proactive respectively.

\subsubsection{System Overheads}

As indicated in Section 4.4.4, the average overhead of an operation is characterized as the average time spent by all the requests during an experiment on performing the given operation. The average overheads due to internal and external communication that were introduced in Section 4.4.4 and the average overhead due to MLE introduced in Section 5.4.4 are used in the context of the hybrid system.

\subsection{Results}

This section focusses on experimental results. In addition to a simulator, the proof of concept prototypes for Systems I-III were run on a system using Amazon EC2 as the public cloud. The results show that the prototype results are similar to the results obtained through the simulation. Both user cost and broker profit are compared for all three systems. In these experiments, the purely reactive system of Chapter 4 is referred as System I-R and 
the purely proactive system of Chapter 5 is referred to as System I-P. The hybrid system is referred to as System I-H.

\subsubsection{Comparison between the Simulator and the Prototype}

Experimental results that capture the variation in Broker Profit $(B P)$ for different values of $f$, or Load Factor, are presented in Figure 6.2. Results obtained from both the simulator and the prototype are presented. For most values of $f$, a difference of $3 \%$ to $5 \%$ was observed between the values obtained from the simulator and the prototype. The better performance of the simulator may be attributed to the assumption of zero communication latencies whereas the communication between the broker and the virtual resources as well as between SCE and the broker in the prototype are subject to non-zero communication latencies with network latencies due to Amazon's internal data center network and external networks having a small impact on broker performance for the prototype. The greatest difference observed is $6 \%$ that was achieved at $f=1$. At lower values of $f$, the difference between the $B P^{a}$ values achieved in both systems is much smaller: for example, a difference of $3 \%$ is observed at $f=0.0$. Using a simulated system is less expensive and faster in terms of execution time in comparison to using a prototype.

Since the difference in the results for the simulator and the prototype is small, the remaining experiments in this chapter have been run using the simulator, thereby saving costs associated with acquiring resources on a public cloud as well as saving time to run the experiments in the cloud environment. Note that the system overheads reported in Section 6.5.6 are based on measurements made on the system prototype. Additionally, a close match between the prototype and the simulator results was found for System II as well, and hence only the simulator results for System II have been presented in this chapter. 


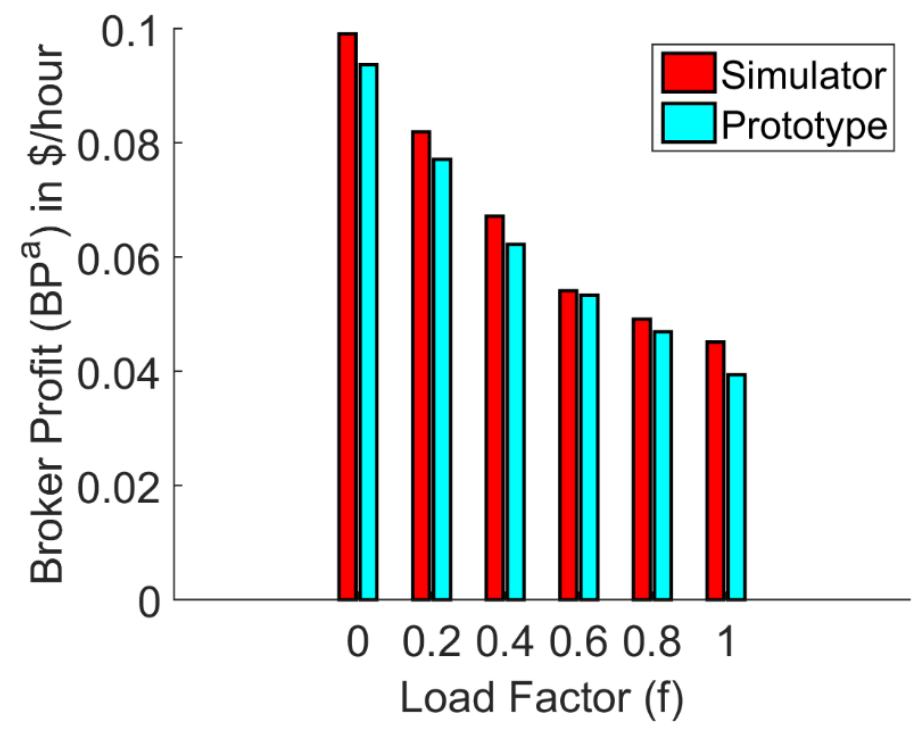

Figure 6.2: Comparison of the Simulator and Prototype for the Hybrid Auto-scaler

\subsubsection{Impact of Load Factor}

Figure 6.3, compares the profit that accrues on System I-H and System II for different values of $f$. In all cases, System I-H is able to generate more profit than System II. The profit is inversely proportional to $f$ : as $f$ increases, profit decreases. For example, when $f$ is $0, B P^{a}$ is the highest. Note that at $f=0$, the system is subjected to only the higher arrival rate. As $f$ increases, the proportion of time for which the system is subjected to the lower arrival rate increases. At $f=1$ the system is subjected to only the lower arrival rate and the profits earned are lower. Overall, for any given $f, B P^{a}$ for System I-H is 3 - 4 times higher than System II (see Figure 6.3). This is due to the fact that an increase in $f$ implies that the broker has to handle a lower number of request arrivals per unit time and thus has a lesser opportunity to accrue a profit by executing a lower number of requests.

Figure 6.4 presents a comparison of the total user cost incurred in all the three systems. As shown in the figure, System I-H leads to a lower total user cost in comparison to the other two systems. The experiments show that an increase in $f$ leads to a lower cost 
per hour for the user. This is due to the fact that an increase in $f$ implies that the broker has to handle a lower number of request arrivals per unit time and is forced to scheduling a lower number of requests, leading to a lower $U C^{a}$.

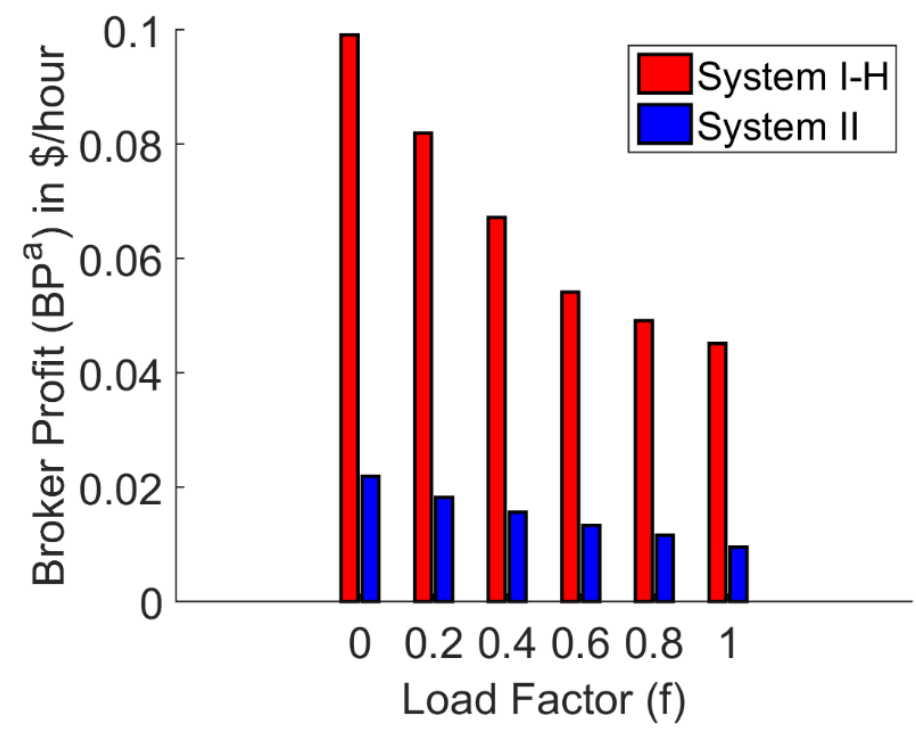

Figure 6.3: Effect of $f$ on Broker Profit for the Hybrid System

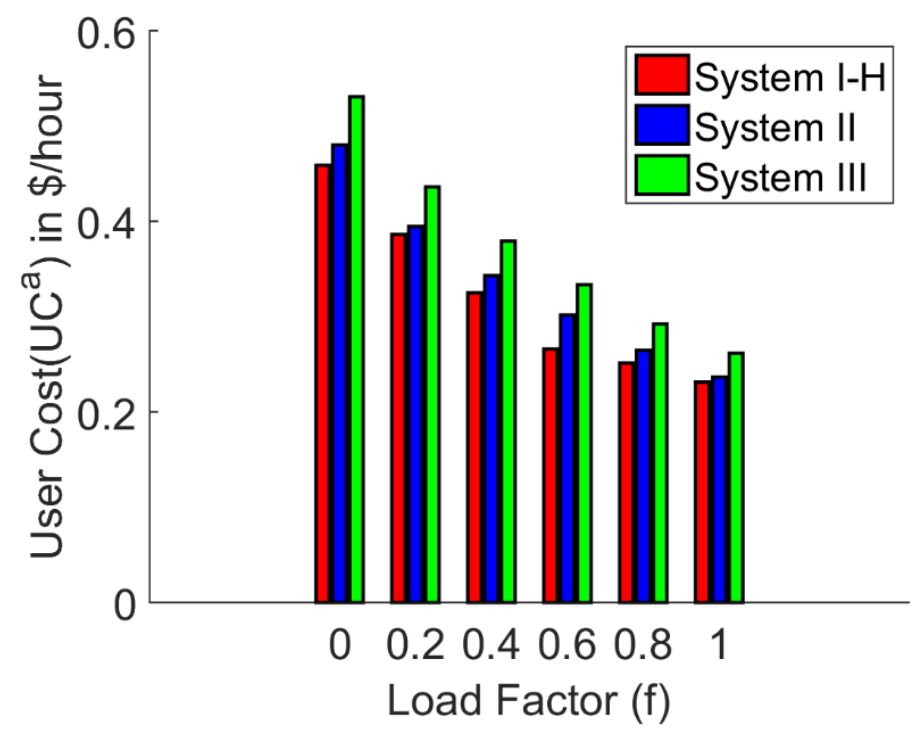

Figure 6.4: Effect of $f$ on User Cost for the Hybrid System

\subsubsection{Impact of Arrival Rate}

A performance analysis of a system subjected to a single arrival rate is performed to 
investigate the impact of $\lambda$ on profit. Figure 6.5, presents the effect of $\lambda$ on the profit generated on System I-H and System II. $B P^{a}$ in both systems is directly proportional to the arrival rate. This is because, as indicated earlier, at higher arrival rates, the system receives a higher number of requests per unit time, thus increasing the potential for earning a higher profit/hour. System I-H earns in between 2 to 6 times the profit earned by System II.

\subsubsection{Impact of Laxity Factor}

In Figure 6.6, Systems I and II are subjected to different values of $L f$. A $L f$ of 0 implies that there is no slack time for the requests. This renders the auto-scaling ineffective because the system cannot schedule multiple requests without missing some of the deadlines. Consequently, the profit accrued is almost 0 . However, as the laxity in the system increases, $B P$ also increases. A $150 \%$ improvement in $B P$ is observed as the $L f$ is increased from 1 to 4. Overall, System I-H performs 3 to 6 times better than System II for a given laxity.

\subsubsection{Impact of Service Time}

Figure 6.7 captures the impact of $S$ on the performance of System I-H and System II. The figure shows that profit increases with an increase in $S$. This is because the larger the service time of a request, the higher the utilization of the resources. This enables the broker to exploit the pricing difference and earn a greater profit at a higher value of $S$. For a mean service time of 12.5 minutes, however, the system accrues almost no profit. Note that for the parameters used in this experiment it would take at least 40 minutes of requests running on a resource per hour for the system to break even and lead to a broker profit. System II follows a similar trend as displayed by System I-H. For any given mean service time between 25 and 75 minutes, broker profit for System I-H is 1.5 to 4 times 
that for System II.

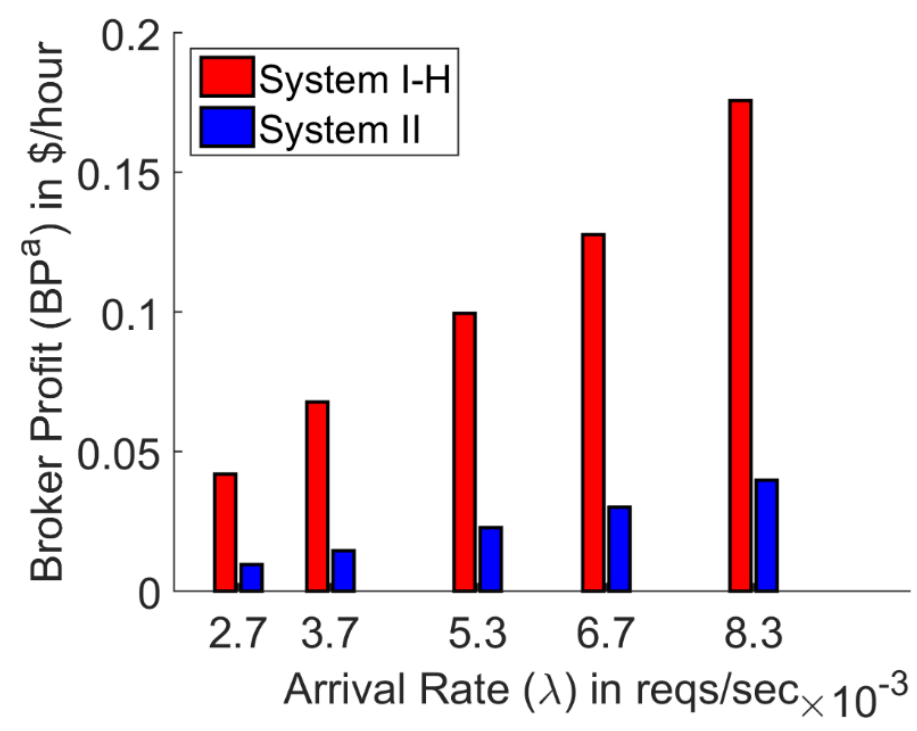

Figure 6.5: Effect of $\lambda$ on Broker Profit for the Hybrid System

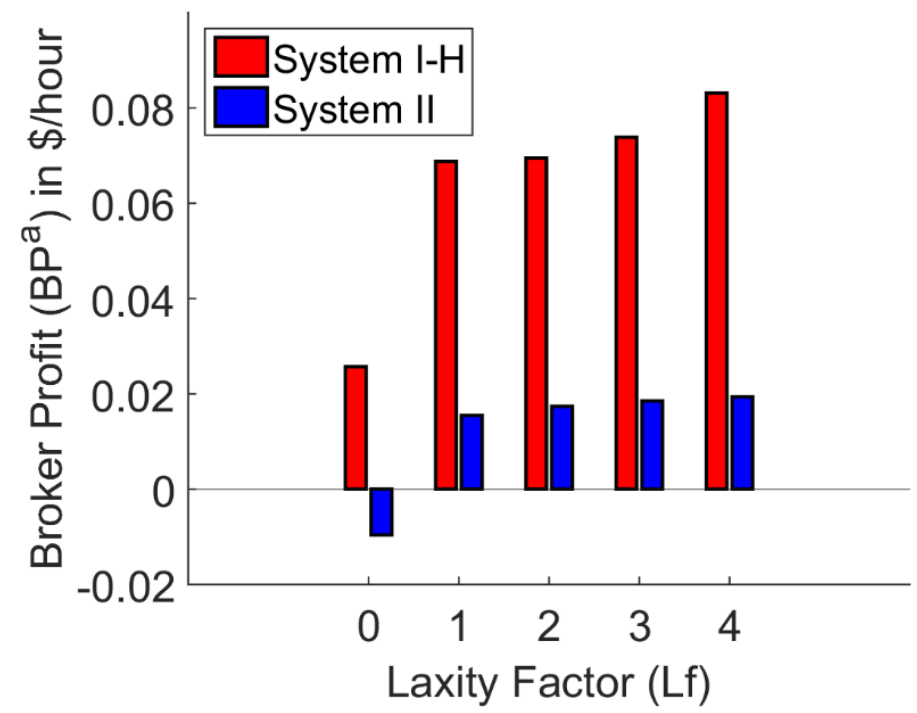

Figure 6.6: Effect of $L f$ on Broker Profit for the Hybrid System

\subsubsection{System Overheads}

An experiment was conducted with all the system and workload parameters held at their default values. The average overhead due to internal communication (defined in Section 4.4.4). is measured to be 59 milli-seconds whereas the average delay due to 
external communication (also defined in Section 4.4.4) is measured at 179 milli-seconds. The average overhead caused due to running the machine learning algorithm (defined in Section 5.4.4) is measured to be 6.3 seconds. The overheads are very small in comparison to the mean service time of a request, which is 50 minutes, or 3000 seconds.

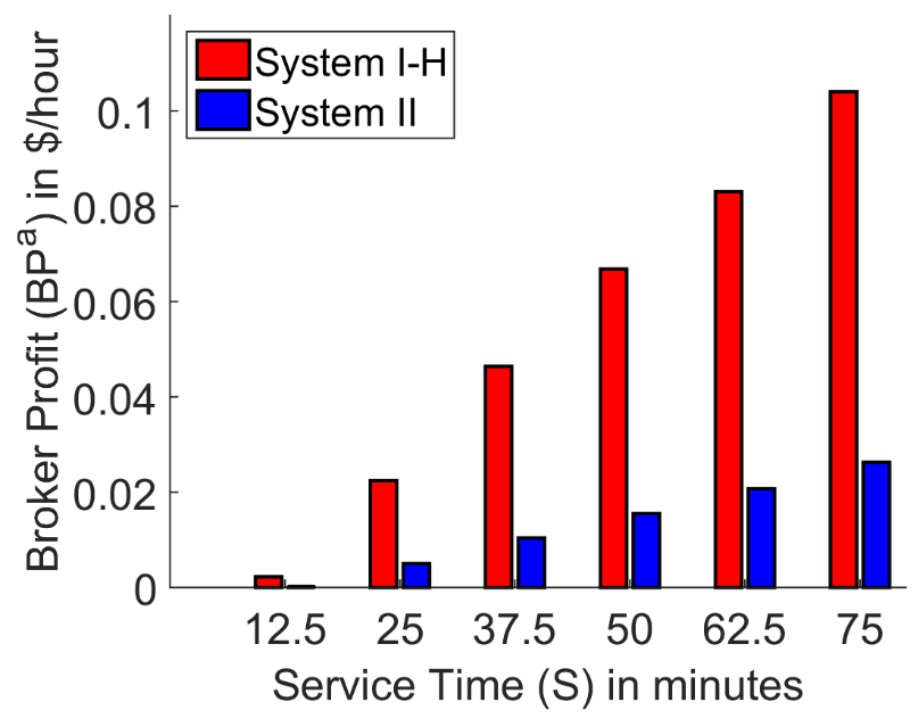

Figure 6.7: Effect of $S$ on Broker Profit for the Hybrid System

\subsubsection{Other Parameters}

This sub-section describes the impact of a number of additional parameters that were varied to analyze the performance of the hybrid system.

\subsubsection{Impact of Number of Requests between Predictions}

Figure 6.8 captures the impact of varying the number of $\mathrm{P}$ upcoming requests predicted on the performance of System I-H. Profit is observed to increase initially with an increase in the value of $P$ used by MLE. However, it reaches a peak at $P=50$ and then decreases to $26 \%$ of the $B P$ as $P$ is increased to 100 . Small values of $P$ do not allow MLE to properly learn the request characteristics. Also, when $P$ is too large, stale information may get included in the prediction process, thus reducing the accuracy of predictions. Thus, 
profit seems to be maximized at an intermediate value of $P$.

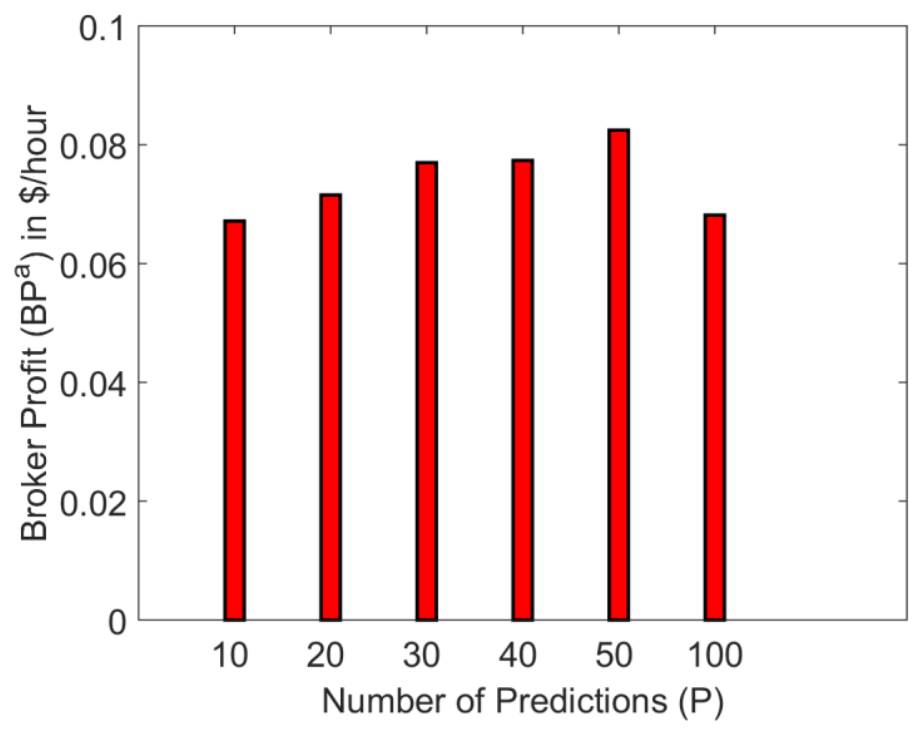

Figure 6.8: Effect of $P$ on Broker Profit for the Hybrid System

\subsubsection{Impact of Machine Learning Algorithm}

The impact of changing the machine learning algorithm on $B P^{a}$ is presented in Figure 6.9. As shown in Figure 6.3, as the load factor increases, the broker profit decreases. Changing the machine learning algorithm while also changing the load factor has the same effect form both machine learning algorithms. For any given value of $f$, the improvement in $B P^{a}$ achieved by SVR over LR is marginal: $2 \%$ to $4 \%$.

\subsubsection{Comparison with Pure Reactive and Pure Proactive Approaches}

This section compares the performance of the hybrid techniques with pure proactive and pure reactive techniques discussed in Chapter 4 and Chapter 5 respectively. The goal of this section is to compare performance metrics: $U C^{a}$ and $B P^{a}$ by for the hybrid autoscaling framework to those achieved with these other frameworks.

In Figure 6.10, $B P^{a}$ for the hybrid system (shown as System I-H), is compared with that of the pure reactive system, System I-R and the pure proactive system, System I-P. 
As in the experimental results presented in Figure 24, as the load factor increases, the broker profit decreases. The graph shows that in almost all cases, the hybrid system performs better than the other two systems. The hybrid system generates up to $11 \%$ and $60 \%$ more profit than System I-R and System I-P respectively.

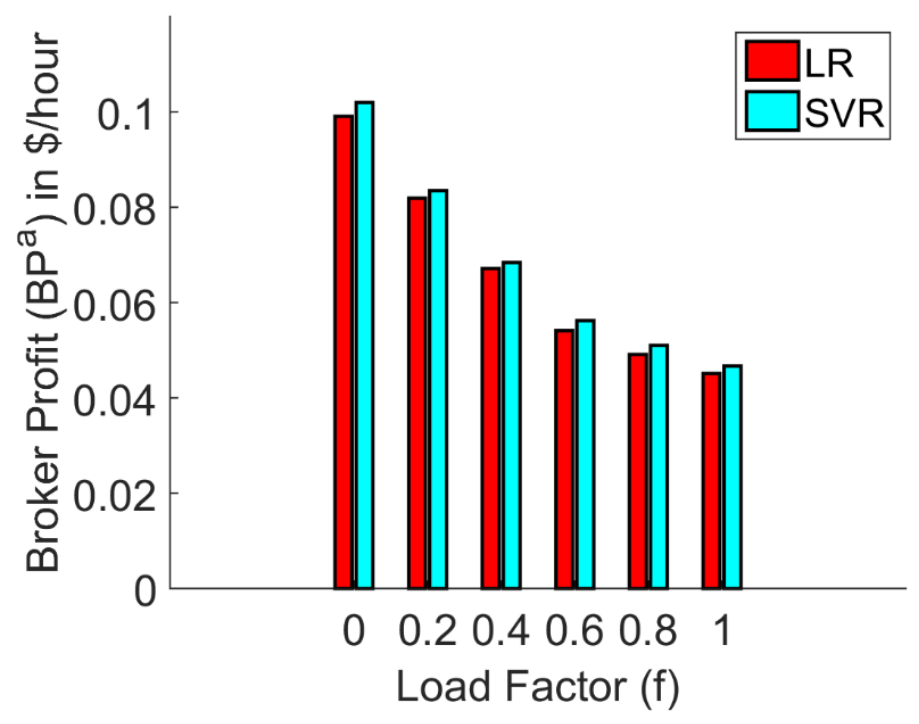

Figure 6.9: Effect of changing the MLA on Broker Profit for the Hybrid System

In Figure 6.11, presents a comparison of $U C^{a}$ for System I-H, System I-R and System I-P. As in case of the experimental results presented Figure 6.4, as the load factor increases, the user cost decreases. In most cases, the hybrid system performs worse than the other two systems. The reason for the hybrid auto-scaler performing worse than System I-R and System I-P is that in the other cases, the brokers accepted fewer number of requests, and led to a lower profit as discussed in Figure 6.10. However, since the hybrid broker for System I-H accepted a higher number of requests per hour, the users had to pay a higher amount to the broker. The hybrid system makes the users pay $7 \%$ and 59\% more than System I-R and System I-P respectively in the worst case. However, the higher cost for the user implies that a higher number of requests are accepted by the 
System I-H in comparison to System I-R and System I-P.

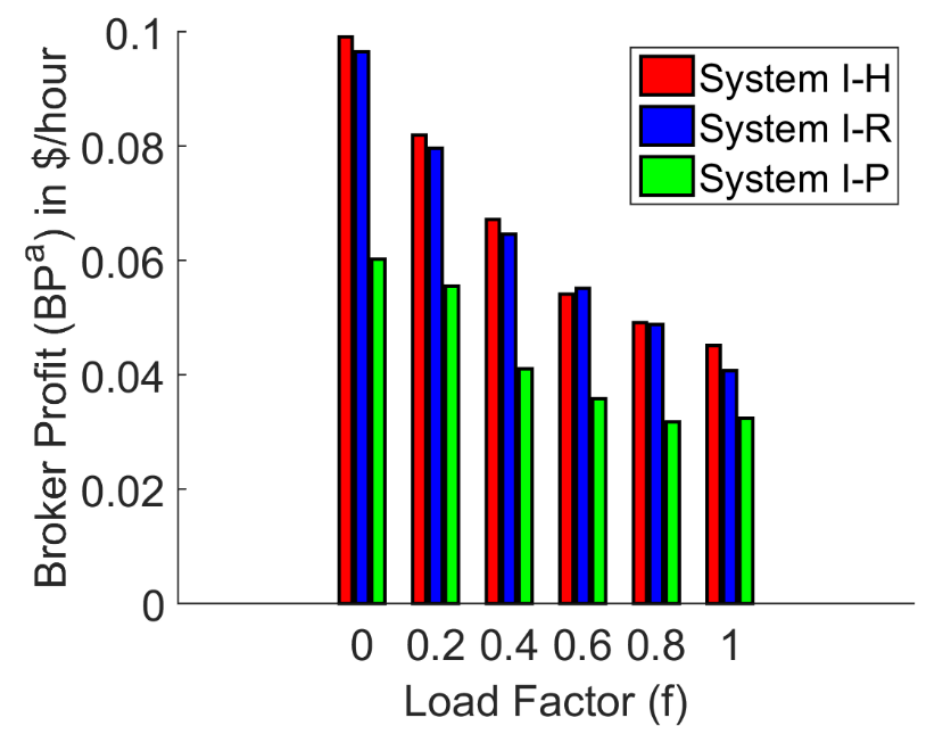

Figure 6.10: Effect of $f$ on Broker Profit

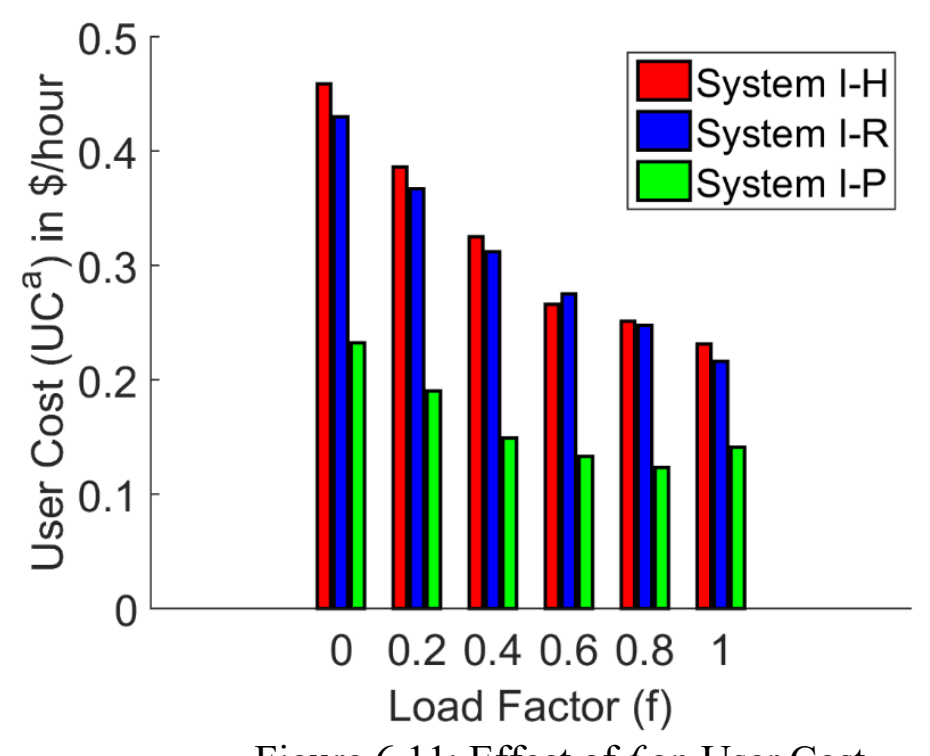

Figure 6.11: Effect of $f$ on User Cost

Figure 6.12, $\mathrm{BP}^{\mathrm{a}}$, presents a comparison of $B P^{a}$ achieved with System I-H, System I-R and System I-P for different values of $\lambda$. As, expected, as the arrival rate increases, the broker profit increases. The graph shows that in all cases, the hybrid system performs better than the other two systems. The hybrid system accrues $5 \%$ and $72 \%$ more profit than System I-R and System I-P respectively. 


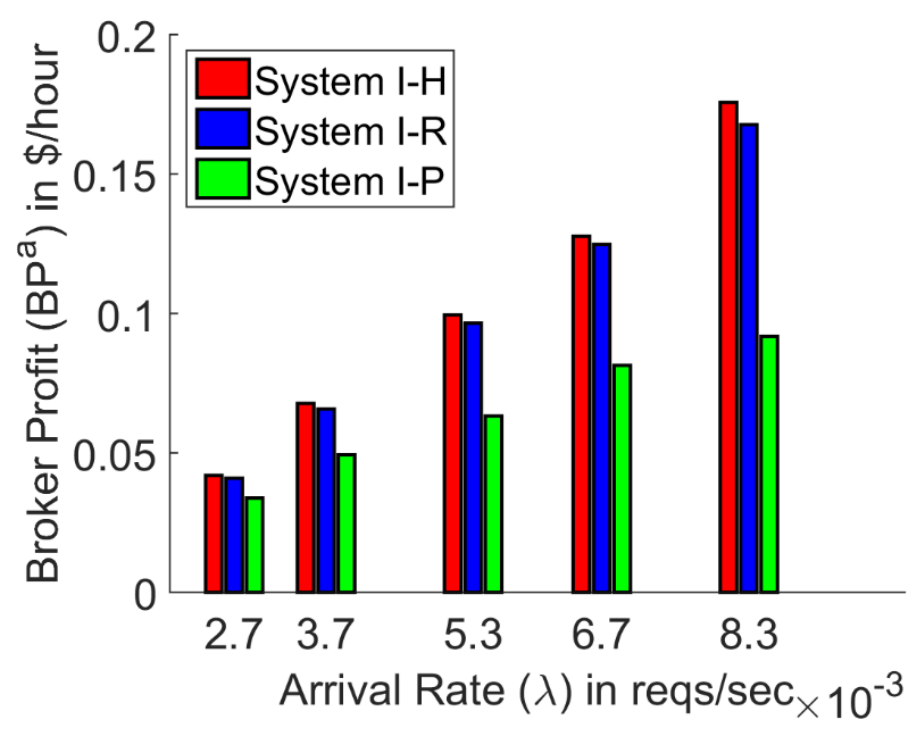

Figure 6.12: Effect of $\lambda$ on Broker Profit

Figure 6.13, $B P^{a}$, presents a comparison of $S$ achieved with System I-H, System I-R and System I-P for different values of $S$. As $S$ increases, the broker profit increases. The graph shows that in almost all cases, the hybrid system performs better than the other two systems. The hybrid system accrues up to 5\% and 321\% more profit than System I-R and System I-P respectively.

Figure 6.14, $B P^{a}$, presents a comparison of $L f$ achieved with System I-H, System I-R and System I-P for different values of $L f$. As $L f$ increases, the broker profit increases. The graph shows that in all cases, the hybrid system performs better than the other two systems. The hybrid system generates up to $13 \%$ and $27 \%$ more profit than System I-R and System I-P respectively. An interesting observation is that in some cases, System I-R performs better than System I-P and worse in other cases. However, the hybrid system, System I-H, always achieves a performance better than the two other systems or close to the best performance achieved for a given value of $L f$. 


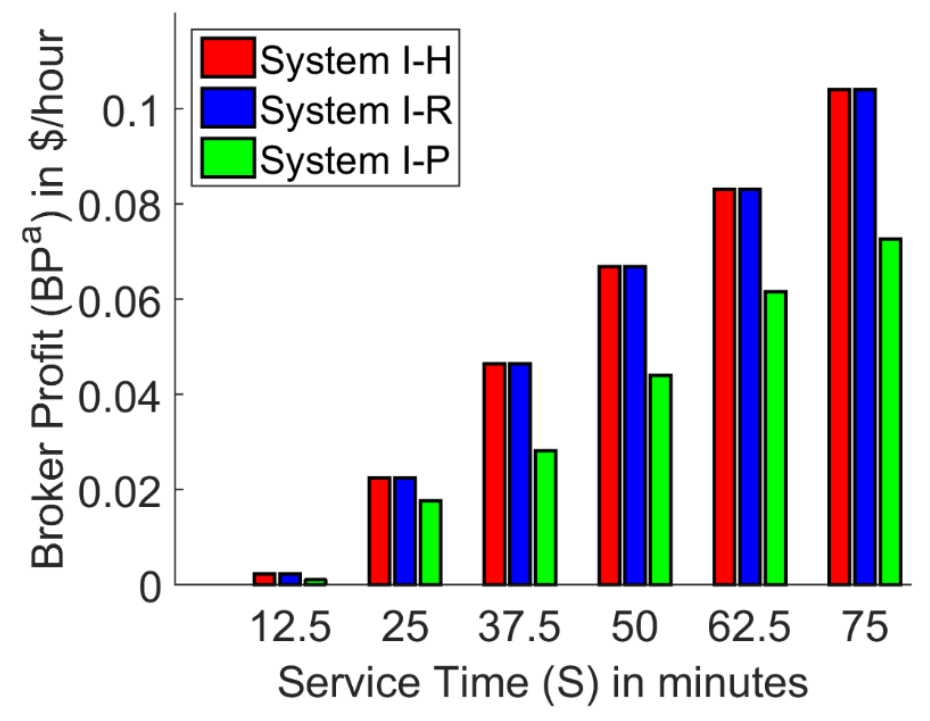

Figure 6.13: Effect of $S$ on Broker Profit

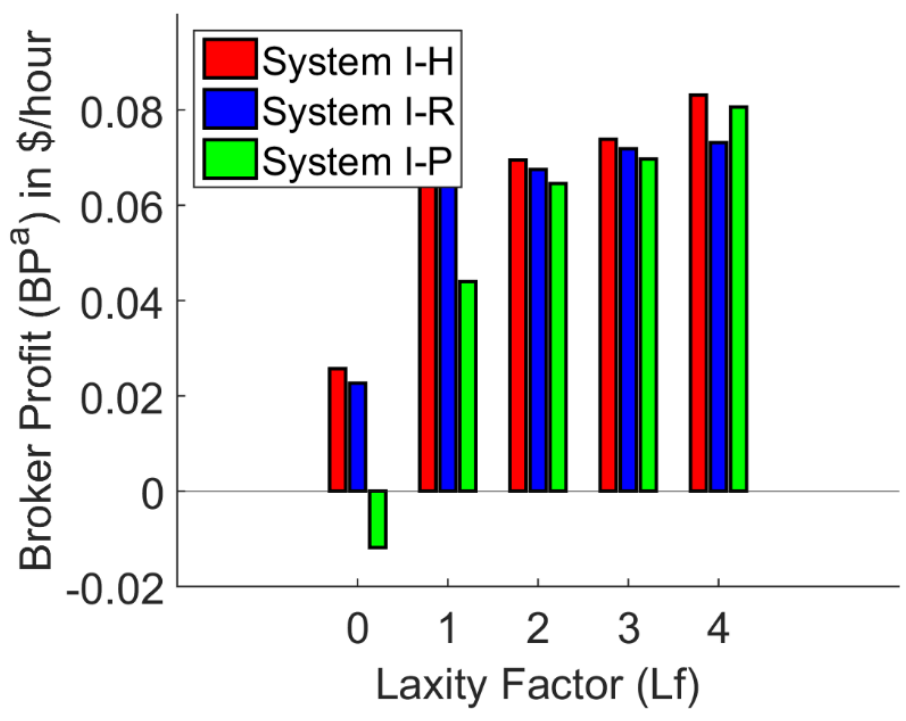

Figure 6.14: Effect of $L f$ on Broker Profit

Additionally, when comparing the three techniques, the proportion of requests rejected, or $B$ is an important metric to consider. Table 6.4 presents the different values of $B$ for the reactive, proactive and hybrid techniques when using the default set of system and workload parameters. The reactive and hybrid auto-scalers reject a much smaller proportion of requests in comparison to the proactive auto-scaler, which may be a reason 
why the proactive auto-scaler accrues lower profits in comparison to the other auto-scalers.

Table 6.4 Comparison of $B$

\begin{tabular}{|l|l|l|l|}
\hline Auto-scaling Technique & Reactive & Proactive & Hybrid \\
\hline$B$ & 0.03 & 0.09 & 0.02 \\
\hline
\end{tabular}

\subsection{Profitability and Cost Analysis}

Two important questions in the context of the proposed Intermediate Enterprise based system concern the relationship between the workload, system and price parameter values that lead to (i) a broker profit and (ii) a lower cost to the user in comparison to the situation in which the user acquires a resource directly from the public cloud provider. Analyses of broker profitability and user cost are presented in this section

\subsubsection{Analysis of Broker Profitability}

This section compares the Profitability (for the broker/IE) Analysis. Let, $t_{r}$ be the time in seconds that a given resource $r$ is used during the period of $T_{r}$ hours, the time for which the resource was "rented" by IE from the cloud service provider).

IE (broker) earning during this period $=t_{r} \times c \_p v t$

The cost incurred by IE for paying the public cloud service provider during this period = $T_{r} \times c \_p u b$

For IE to remain profitable for a given resource $r$, broker earning must exceed the cost incurred by the broker:

$$
\begin{aligned}
& t_{r} \times c_{\perp} p v t>T_{r} \times c_{\perp} p u b \\
& \text { - } \frac{t_{r}}{T_{r}}>\frac{c_{p u b}}{c_{p v t}}
\end{aligned}
$$

Dividing both sides by 3600 implies: 
- $\frac{t_{r}}{T_{r} \times \mathbf{3 6 0 0}}>\frac{c_{p u b}}{c_{p v t} \times \mathbf{3 6 0 0}}$

- $\boldsymbol{U}_{\boldsymbol{r}}>\frac{c_{-} p u b}{c_{-} p v t \times \mathbf{3 6 0 0}}$

Where $U_{r}$ is the utilization of the given resource $r$. Thus, if $U_{r}$ for every resource exceeds $\frac{c \_p u b}{c_{p v t} \times \mathbf{3 6 0 0}}$, IE is guaranteed to remain profitable. But this bound is conservative (i.e. IE may remain profitable even if $U_{r}$ for some resources do not satisfy the inequality). Hence, to determine the conditions under which the overall profit is greater than zero, a new inequality that leads to a tighter bound is formulated:

$$
\begin{array}{ll}
\text { - } & \sum_{r=1}^{R}\left(t_{r} \times c_{\perp} p v t\right)>\sum_{r=1}^{R}\left(T_{r} \times c \_p u b\right) \\
\text { - } & \frac{\sum_{r=\mathbf{1}}^{R}\left(t r \times c_{\_} p v t\right)}{\sum_{r=\mathbf{1}}^{R}\left(T r \times c_{-} p u b\right)}>1
\end{array}
$$

Note that the broker will not remain profitable if this inequality is not satisfied

\subsubsection{Analysis of User Cost}

Let, $s_{r}$ be the time in seconds that a given resource $r$ is used by request $s$ during the period of $T_{r}$ hours.

For the price model to be attractive to the user: User cost (with IE) must be lower that the User Cost (direct) (the cost incurred when the resource is acquired directly from a public cloud provider)

$$
s_{r} \times c \_p v t>\left(\left\lceil\frac{\mathbf{s}_{\mathbf{r}}}{\mathbf{3 6 0 0}}\right\rceil \times c \_p u b\right)
$$

The user can use this inequality to determine which system is better for executing the request: the IE based system or the public cloud provider. For example, with the defaults values of $c \_p v t$ ( $\left.\$ 0.00000833 / \mathrm{second}\right)$ and $c \_p u b$ are $(\$ 0.02 /$ hour) used by the IE based system, it remains more attractive as long as $s_{r}<2400$ seconds. For a higher ratio of $c \_p u b$ 
and $c \_p v t$, with $c \_p v t=\$ 0.00000694444 /$ second and $c \_p u b=\$ 0.02 /$ hour for example, the IE based system will be more attractive to the user if $s_{r}<2880$ seconds.

\subsection{Observations}

As demonstrated through simulation experiments and measurements made on a prototype, for a range of workload and system parameters experimented with the proposed framework can generate a profit for the intermediary enterprise hosting the broker while reducing the cost incurred by users. The hybrid auto-scaling technique introduced in the chapter combines a machine learning based proactive approach with a reactive approach for scaling resources. The chapter also discusses the various overheads associated with the hybrid auto-scaling system and demonstrates that these overheads are low. The key feature of the framework is presented:

- The novel hybrid auto-scaling technique introduced in this research combines a proactive approach by utilizing machine learning algorithms and a reactive approach that scales resources based on a Grade of Service criterion.

A number of insights were gained by performing simulation experiments. Some of the important observations are presented:

- The experimental results demonstrate that using the proposed hybrid broker can lead to a higher profit as compared to other proactive and reactive systems. The broker profit is observed to depend on parameters, such as load factor, arrival rate, mean service time, laxity factor as well as the number of requests between predictions.

- A higher mean arrival rate enables the broker to earn a higher profit. Additionally, lower the load factor, higher is the difference between the profit generated by the 
hybrid system and that generated by System II that leads to better performance for the hybrid system.

- The broker profit is observed to be directly proportional to mean service time.

- The broker profit seems to increase with the increase in the laxity factor. Most laxity factors experimented with lead to a broker profit. However, at a laxity factor of 0 , the requests have no slack time to meet their deadlines. In such a situation, the profit earning mechanism fails.

- As in the case of the pure proactive system disused in Chapter 5, changing the machine-learning algorithm from linear regression to SVR has a small impact on the broker profit. However, the additional computation time for using a more compute intensive algorithm increases the system overhead considerably.

- The hybrid system performs better by accruing more broker profit than a purely proactive or purely reactive system in most cases experimented with, each of which corresponds to a given combination of system and workload parameters. As a consequence of accruing more broker profit, the hybrid system, in most cases, charges the user more than the pure proactive and pure reactive systems.

- The reactive broker performs better than the hybrid broker in a few cases, for example, when the load factor is at 0.6 . The reactive broker also performs better than the proactive broker in all cases except when laxity factor is kept at 4 . The hybrid broker always performs better than the proactive broker. 


\section{Chapter 7 Extending the Auto-Scaling Approach to Network}

\section{Resources}

The auto-scaling techniques introduced in the previous chapters deal with scaling only compute resources. The objective of this chapter is to discuss the extendibility of the auto-scaling techniques to allow scaling both compute and network resources in a cloud environment. In line with the techniques described in the previous chapters, auto-scaling operations will be performed using a reactive approach that was used in Chapter 4. Only a discussion of how to extend the reactive auto-scaling technique described in Chapter 4 to handle network resources is presented in this chapter. A complete investigation for autoscaling network resources using other auto-scaling techniques introduced in this thesis, such as proactive and hybrid techniques is not performed in this thesis. Section 7.1 introduces a traditional Data Center Network (DCN) architecture while Section 7.2 describes the broker architecture to allow the system to control network resource, in addition, to compute resources by introducing new components that are required to achieve network auto-scaling. The auto-scaling algorithm is introduced in Section 7.3 while the system and workload parameters that are used to analyze the performance of the technique are described in Section 7.4. Section 7.5 presents the results of the experiments for analyzing system performance while Section 7.6. states the observations from performing the experiments.

\subsection{Introduction}

Most research presented in the thesis has focused on investigating techniques that auto-scale only compute resources. This is due to the ubiquitous availability of compute resources, and also because cloud providers readily provide mechanisms to provision and 
de-provision compute resources. Network resources, on the other hand, are not offered by any service providers as on-demand resources that may be rented publicly. Other researchers have started looking at network resources as a candidate for auto-scaling, and some of the research on network auto-scaling has been discussed in Section 2.5. This chapter focusses on demonstrating the reactive auto-scaling technique introduced in Chapter 4 to handle both compute and network resources simultaneously.

One of the challenges when handling network resources in the cloud is understanding how the network resources are organized in the data center of a cloud provider. A traditional Data Center Network (DCN) connects virtual servers [63] to deliver services to cloud end-users. In such a cloud, the DCN must be able to meet demands for transferring data to compute resources running applications. The network can become a bottleneck for computing on such a system [63]. Such bottlenecks arise due to traffic patterns inside a Data Center (DC). Traffic in the DC generally flows in three directions [15]. North-South traffic is limited to traffic that enters and exits the DC. This traffic crosses the DC boundary using the edge firewalls and/or routers and communicates over the Internet. Hence, most DC security solutions focus on handling this type of traffic. EastWest traffic, on the other hand, flows between DC resources and applications and never leaves the DC. Finally, Inter-DC traffic flows between multiple DCs, and between DCs and the private/public cloud. The work in [15] introducing Cisco's Global Cloud Index says that, unlike in campus networks, the dominant volume of traffic in the DC traverses in an East-West direction (76\%), followed by North-South traffic (17\%), and finally, interDC traffic, which currently accounts for only $7 \%$ of the total traffic. The high percentage of East-West traffic indicates that more network traffic is flowing inside a data center and 
may be attributed as the primary cause for the DCN bottlenecks. With applications based on a distributed computing model, minimizing the completion time of application requests depends on moving data using the DCN in a timely manner. This chapter aims to introduce techniques to reduce the East-West traffic by reducing the average number of links over which network traffic needs to traverse. East-West traffic is the network traffic flowing within DCN and is introduced in Section 1.1.4.3.

DCN is the communication infrastructure used in a data center and is described by the network topology, routing/switching equipment, and the communication protocols (e.g., Ethernet and IP). Figure 7.1 shows a conventional data center network topology. In this topology, the Top-of-Rack (ToR) switch in the access layer provides connectivity to the servers mounted on every rack. Each aggregation switch (AS) in the aggregation layer (sometimes referred to as the distribution layer) forwards traffic from multiple access layer (ToR) switches to the core layer. Every ToR switch is connected to multiple aggregation switches for redundancy. The core layer provides secure connectivity between aggregation switches and core routers $(\mathrm{CR})$ connected to the Internet.

Real data centers may have 1000 s of servers with close to $100,000 \mathrm{VMs}$ and numerous routers and different levels of the network topology. However, Figure 7.1 tries to present this topology on a smaller scale. Each server is connected to a Top of Rack (ToR) router which connects to the aggregation router (AR). The AR connects to the core router (CR). The CR has an interface to the WAN and the Internet and allows the flow of NorthSouth data. The techniques discussed in this chapter are dependent on the architecture of the network having multiple layers. Using other DCN architectures will require a different cost function to enable the broker to accrue a profit. 


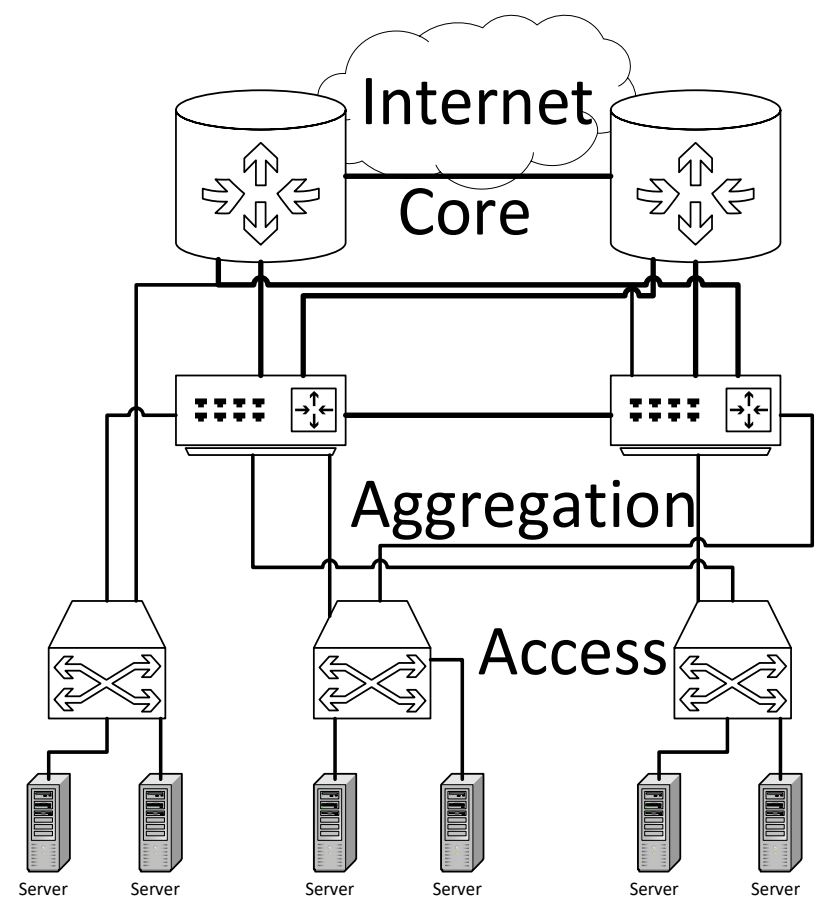

Figure 7.1: Traditional Data Center Topology

\subsubsection{Supporting Technology for enabling auto-scaling network resources}

An important requirement for network auto-scaling performed in the algorithm described in Table 7.1 is the ability to handle bandwidth reservation. A challenge when dealing with traditional DCNs is that there is no performance isolation between different requests using the same network links. Performance isolation is important for achieving bandwidth reservation. While isolation can be achieved by enforcing strict rate limits on certain types of traffic, this leads to inefficient use of DCN because the traffic is not present at a steady rate. Current DCN operators use admission control that works with the Transmission Control Protocol (TCP) to allow network requests to share the network bandwidth using a strategy known as max-min fair sharing [64]. However, max-min fair sharing at the TCP level is not the appropriate model for sharing data center services. This is because certain network requests cannot be given priority over other requests and some selected network requests may get an unfair share of the bandwidth. 
To share the network bandwidth among multiple network requests, DCN operators may partition the network bandwidth over the network links that connect the internal routers. Partitioning the network bandwidth forms another important consideration for achieving bandwidth reservation. Such partitioning aims at creating multiple virtual networks (VNs) on top of a shared physical network allowing each VN to be implemented and managed independently. By separating logical networks from the underlying physical network, it is possible to introduce customized network protocols and management policies. Also, since VNs are logically separated from one another, implementing performance isolation and application QoS is facilitated. Management procedures of VNs will be more flexible because every VN has its own control and management system.

Developing new technologies for addressing these issues has started receiving attention from developers of certain cloud platforms that allow users to manage the network infrastructure. An example is the open-source cloud platforms known as OpenStack, that allow users to control network resources used in the Neutron project [18]. Public cloud providers that decide to build their cloud infrastructure with OpenStack in the future may allow end-users API access to reserve network bandwidth by charging network reservations on a pay-as-you-go basis, similar to renting out compute instances. Note that many VMs are hosted by a Hypervisor, in which providing bandwidth guarantees from one VM to another is still not possible using OpenStack. Neutron only provides bandwidth reservation from one host to another host. Some VM to VM bandwidth reservation techniques has been discussed in literature [65] and implemented in real-world scenarios.

The ability to reserve bandwidth becomes more useful when systems can satisfy the QoS of network service after reserving network bandwidth. Moreover, keeping cost 
low while satisfying the QoS is challenging inside a data-center. One of the challenges is related to the dynamic nature of the workload the systems is subjected to. The network traffic can also be bursty and the resources in a data-center need to handle the peak traffic without breaking the SLAs associated with the user requests. Service providers should make a prompt decision to acquire additional cloud resources to meet higher demands and avoid QoS degradation or further system corruption. The algorithm presented in this chapter in Section 7.3 is directed at addressing this challenge and the algorithm assumes that network bandwidth is reserved.

In addition to providing bandwidth guarantees for each request, the algorithm described in Table 7.1 is directed at reducing the East-West traffic in the DCN and meet the SLA specified by the user request while attempting to minimize the time to complete the request, thereby saving on user costs. These benefits will be shown experimentally in Section 7.5.

\subsection{System Architecture}

The overview of the system is given in Figure 7.2. Readers may compare this to Figure 3.1 presented in Chapter 3. One of the key differences between Figure 3.1 and Figure 7.2 is the introduction of a network topology of the Internal Resource Manager (IRM). The role of the component is to accept control requests from the broker using the DRPM module and reserve bandwidth for user requests. IRM achieves network isolation by creating logical networks inside the DCN. The cloud provider guarantees the performance isolation for any data being transferred by separate requests. VMs inside a single server can communicate without any network cost as communication occurs internally without using physical network links. To communicate with VMs in a different 
server, the VMs must use network links inside the data center. VMs within a single aggregation router can communicate using two hops, one from the source VM to the source ToR to the aggregation router and the other from the aggregation router to the destination ToR and finally to the destination VM. Communication between two VMs using a single ToR is not considered as a communication cost because the ToR is directly connected to the server hosting the VMs, involving a fixed overhead which does not affect traffic inside the DCN. Finally, communication that occurs between VMs belonging to separate aggregation routers needs four hops, as traffic needs to flow to the core router and then back to the destination VM.

The broker component is similar to the other brokers described in previous chapters along with additional internal components to handle network resources. A request is sent by the SCE and scheduled by the broker on one of the resources acquired from the cloud. To keep the broker consistent with previous brokers, the broker can perform no additional functionalities to control the data center running resources in the cloud. In order to maximize the profit for the broker as well as reduce costs for the user, the broker needs to reserve the network bandwidth by allocating network share of a link to the request.

The components of the broker are shown in Figure 7.2. Components such as the request handler, MMS, GoS and DM have been described in Chapter 3. The broker includes a new component called the network controller to compute the cost of transferring data between to the storage resource and the VM where the request is executed. The broker needs to query the current state of the data center with respect to servers and network topology and the core, aggregation and ToR levels. The broker also requires the current capacity of the DCN to compute a network path over multiple links to minimize the East- 
West traffic. The capacity of each network link in the DCN is provided by IRM, which communicates with the cloud provider, and relays the current DCN usage to the Decision Maker (DM).

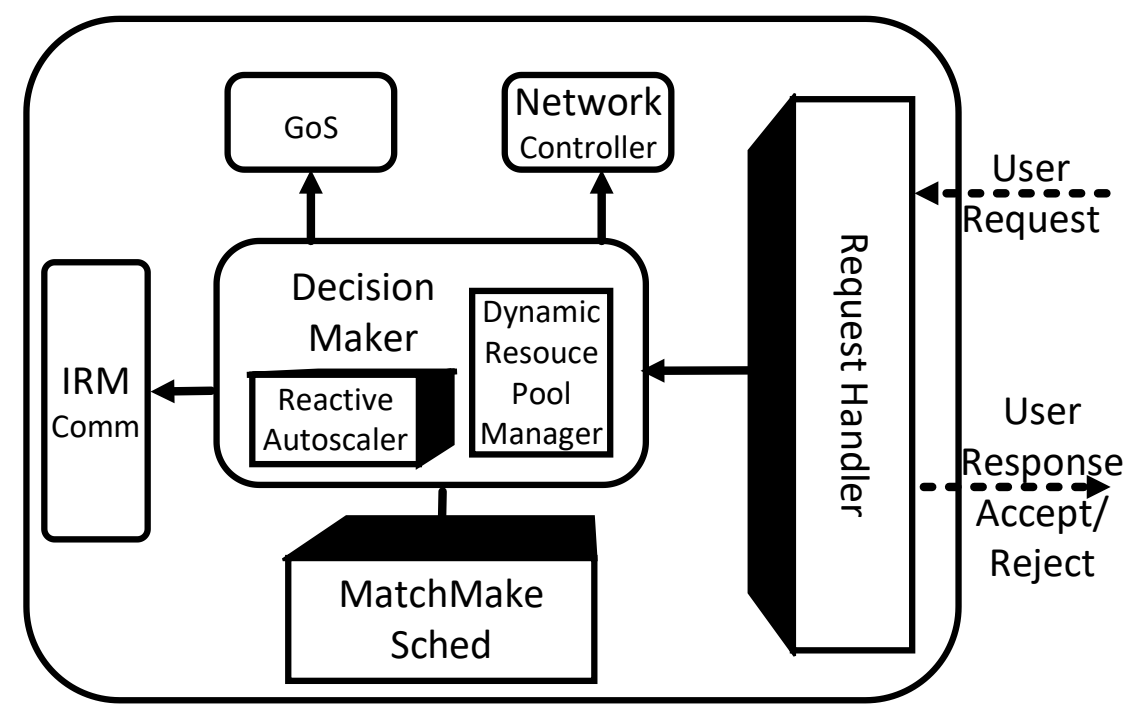

Figure 7.2: Broker Architecture for Handling Network Resources

\subsubsection{Request Handling and Updated Pricing}

Although the broker pricing model has been described in detail in Section 3.2, this chapter revisits the concept in order to address the pricing for network resources. The goal for the broker, as with other chapters, is to maximize the profit for the VPC provider (intermediary enterprise) while attempting to reduce the cost for the client and meeting the desired SLA. The broker continues to rent resources from the public cloud provider by paying $c \_p u b_{\text {compute }}$ per hour and rents out compute resources to SCE at $c \_p v t_{c o m p u t e}$ per second for representing the compute costs for the broker and SCE respectively. However, the broker also deals with auto-scaling network resources, which it leases from the cloud provider in a similar manner to compute resources. The network resources are priced in terms of the dollar amount per $\mathrm{Gb}$ per time interval. The cloud provider charges the broker c pub $b_{\text {network }}$ per $\mathrm{Gb}$ per hour. The broker charges the user $c$ p pvt network per second. These 
amounts need to be appropriately chosen such that user charge is considerably more per hour than $c \_p u b$. Otherwise the broker may accrue a loss. Since the total user cost incurred by the client enterprise is also the earning for the intermediary enterprise provider (broker), the broker profit is calculated with the same equation as given in (1).

Users submit requests to the broker. The requests comprise of compute as well as network demands. User requests are characterized by AT, EST, ST, DL and T, already described in Section 3.1.2. To allow users to specify the network requirements, each request also specifies a list of data stores from which data must be transferred to the compute resource before the execution of the request can start. Data stores (DS) are resources in a data center that offer an API to transfer requested data to a virtual machine. Typical examples of data stores can be database systems or network storage devices.

There are two phases in the data transfer between the compute resource where the request would be executed and the various DS, spread across the data center. An init phase and a final phase. An init phase characterizes the initial data transfer phase. This phase allows the request to specify a set of DSs and the amount of data to be retrieved from each DS, characterized by a network demand. Each of the network demands specified inform the broker to use the DCN links to transfer data from a DS to a compute resource where the request will execute. The broker needs to calculate the most economical network path to transfer the data. Once the execution of the request has completed, the broker transfers data back to one of the data stores. This is characterized by the final phase. This phase allows the request to specify one DS to which the computed data is sent back to, from the compute resource. The request must also contain the following information:

- Network Demand (ND) - The location of data stores in each phase as well as the 
amount of data to be transferred to and from the data store. For representing the bandwidth requirements of each request, a Tenant Application Graph (TAG) model has been used.

The Tenant Application Graph (TAG) is a model proposed by [66] which is essentially a directed graph that cloud users can use to describe bandwidth requirements for applications. This work uses the TAG abstraction to model the actual communication patterns of requests. Users request specify a bandwidth $B$ between a storage and a compute node. This is modelled by placing a directed edge between the corresponding vertices in the TAG model. Each directed edge $e=(u, v)$ from say, a compute resource u to a storage resource $\mathrm{v}$ is labeled with an ordered pair $\langle u, v>$ that represents network demand. The init phase specifies multiple DS and the amount of data to be transferred to a compute resource. Consequently, the demand for this phase is expressed as a collection of multiple TAGs, where the destination VM is always consistent whereas the source DS may change based on the network request requirements. The final phase has only one TAG associated with it since this thesis assumes the result needs to be sent back to only one data store.

Public cloud providers like AWS have not adopted alowing end-users to request a portion of the bandwidth. However, this paper [67] formulates a mathematical model for leasing network resources. The network resource leasing by the broker takes place in one-hour increments. This is similar to model used by Telecom providers whereby they lease out the use of their network to smaller companies for a time period. For a given set of $k$ requests with service times $\left\{\mathrm{c}_{1}, \mathrm{c}_{2}, \ldots, \mathrm{c}_{\mathrm{k}}\right\}$ seconds, and total data transferred $\left\{d_{1}, d_{2}, \ldots, d_{k}\right\}$, where the $k^{t h}$ request generate $j$ link demands $\left\{l d_{1 k}, l d_{2 k}, \ldots, l d_{j k}\right\}$. Each link demand specifies the data transferred from one network link to another. For data to be transferred from one DS 
to a compute resource, one or more network links must be used.

The cost savings per request is calculated by subtracting the amount the user would pay the broker to service the request from the amount the user would need to pay if they were to service the request by renting resources directly from the cloud provider. Summing up the saved amounts for all requests gives the total cost savings and is expressed as given by: Total Cost Saving $(C S)=$

$$
\begin{gathered}
\sum_{i=1}^{k}\left[\left(\left\lceil\frac{c_{i}}{3600}\right\rceil \times c_{-} p u b_{\text {compute }}-c_{i} \times c_{-} p v t_{\text {compute }}\right)+\sum_{l=1}^{j}\left(\left\lceil\frac{l d_{l}}{3600}\right\rceil \times\right.\right. \\
\left.\left.c_{-} p u b_{\text {network }}-l d_{l} \times c_{-} p v t_{\text {network }}\right)\right]
\end{gathered}
$$

The broker controls $N$ resources at a given point in time: $\operatorname{Res}=\left\{\operatorname{Res}_{1}, \operatorname{Res}_{2}, \ldots \operatorname{Res}_{N}\right\}$, where $N$ changes dynamically with user demand. The broker also controls a portion of the network links and a slice of their bandwidth at a given point of time: $\mathrm{Net}=\left\{\mathrm{Net}_{1}{ }^{\mathrm{B1}}, \mathrm{Net}_{2}{ }^{\mathrm{B2}}, \ldots\right.$ $\left.\operatorname{Net}_{Q}{ }^{B Q}\right\}$, where $Q$ also changes dynamically. $B=\left\{B_{1}, B_{2}, \ldots B_{Q}\right\}$ are the bandwidths reserved by the broker on links 1,2,.. Q respectively. Once requests have been scheduled, they cannot be cancelled from the scheduler. During the scheduling of the request, the broker decides on the network path chosen by the request TAG requirements and determines a final price of the request for the broker. Thus, the broker guarantees the execution of a request which has been accepted by the system, within the specified deadline.

\subsubsection{Matchmaking and Scheduling}

The MMS component used by RH and by DM is similar to that described in previous chapters (see Section 3.1.4 for more details). The matchmaking component, however, uses a best fit $(\mathrm{BF})$ strategy instead of the First Fit (FF) for matchmaking and an Earliest Deadline First (EDF) strategy for scheduling. This is different from the previous chapters 
in terms of matchmaking since previous work did not have an incentive to select one compute resource over another. This is because the chapter also focusses on reducing the amount of East-West traffic flowing through the DCN. Selecting a compute resource that reduces the number of links over which the data traverses helps achieve this goal. The best fit algorithm scans resources acquired by the system sequentially and allocates the request to the resource capable of handling the request with the minimum number of network links utilized to transfer data to the compute node from the DS. Each compute resource currently acquired by the broker is considered by the matchmaker to determine whether the current request can be scheduled on one of those resources. Once a resource is selected, scheduling the request on the resource is performed using EDF, which is described in Section 3.1.4.

\subsubsection{Decision Maker}

If MMS accepts the request, it is scheduled to be executed on the selected resource at the time computed by MMS. However, if the request cannot be scheduled in the existing resources by MMS, DM invokes the Reactive Auto-scaler (RA) to decide whether the request, after a new resource is acquired, would be profitable for the broker. For a request $i$, the broker needs to calculate whether the request accrues a profit for the broker or not. The request has a service time of $c_{i}$ seconds, and $d_{i}$ is the total time for which all network requests are transferred generating $j$ link demands $\left\{n d_{1}, n d_{2}, \ldots, n d_{j}\right\}$. To determine whether the request is profitable, RA checks the broker profit for the $i^{\text {th }}$ request against the following condition:

$$
B P_{i}>0
$$

The condition determines whether the amount being paid to the public cloud to reserve the compute resource as well as all the network links is lower than the amount being 
charged to the End User by the broker.

Here,

$$
\begin{gathered}
B P_{i}=G \times\left[\left(c_{i} \times c_{p v t_{\text {compute }}}\right)-\left(\left\lceil\frac{c_{i}}{3600}\right] \times c_{\text {pub } b_{\text {compute }}}\right)\right] \\
+\left[\sum_{l=1}^{j}\left(\left\lceil\frac{n d_{l}}{3600}\right\rceil \times c_{-} p u b_{\text {network }}-n d_{l} \times c_{-} p v t_{\text {network }}\right)\right]
\end{gathered}
$$

$G$ is a constant greater than 1 that allows the system to account for the broker's ability to produce an earning that is more than that accrued from only servicing the current request by time-sharing the compute resources amongst multiple requests. Since the broker operates by sharing the acquired resources with other requests, $G$ is introduced to account for the possibility of the resource being utilized by other requests in the time remaining to earn a higher amount of money. The broker profit is computed by first analyzing the amount paid by the broker to the cloud provider for reserving the compute resource and its associated network resources in order to satisfy the $i^{\text {th }}$ request. This amount is then subtracted from the amount paid by the user for handling the request to determine the broker profit for the $i^{\text {th }}$ request.

The decision maker computes the $\mathrm{j}$ link demands by iterating over each TAG in the init and final phases for the request's ND. Each TAG requirement may be met by selecting several possible network link combinations from the compute resource to the storage resource. Moreover, the decision maker can also decide to select a compute resource from a list of compute resources under the broker's control. The decision maker decides to minimize user costs by selecting the compute resource that uses the least number of links to transfer data between the compute and storage resources. Hence, if the decision maker can schedule the request on two different compute resources under the broker's control, it will select the compute resource with the least number of network link traversals. This 
ensures the minimization of East-West traffic in the $\mathrm{DCN}$, one of the goals of this paper, by placing the compute request on a compute resource near the storage resources.

\subsubsection{Internal Resource Manager}

The Internal Resource Manager (IRM) is a module running inside the broker that communicates with the network controller of a DCN to provide a more detailed understanding about the data-center resources and DCN topology in order for the broker to make a decision regarding the leasing of network resources. IRM possesses information about all the links in the DCN with their allocations and bandwidth guarantees which helps in deciding to accept or reject a request. To allow such leasing operations of network resources, there exists APIs from the various open standards and vendors (OpenStack, OpenFlow, VSphere, NSX etc.). However, it is still challenging for enterprises to build applications that can leverage a heterogeneous data-center infrastructure. Current public cloud providers like Amazon's AWS and Microsoft's Azure do not provide an API to lease network resources, although they have implemented and exposed APIs to rent compute and storage resources. For the technique presented in this paper to be viable, the broker would need to lease network resources from the public cloud provider. The broker should also be able to retrieve the DCN topology which will allow it to determine the appropriate links from the public cloud provider. The IRM Comm. module inside the broker implements a communication module for communicating with IRM to make network link acquisition decisions.

\subsection{The Auto-scaling Algorithm with Network Resources}

This section introduces the auto-scaling algorithm that is similar to the reactive auto-scaling algorithm used for compute resources with some differences. As network 
resources are introduced, the algorithm must account for these resources as well as the time at which they have been acquired and must be released. The other major part of the algorithm involves deciding which links to reserve. In the previous algorithm where only compute resources were involved, the cloud provider assigned a compute resource when requested. However, in this case, there may exist many link paths, only a few combinations of which minimize East-West traffic and thereby reduce user cost. Finally, the decision to select a compute resource to execute user requests depends on reducing East-West traffic by reducing the number of links. These differences need to be accounted for in the modified algorithm when auto-scaling compute and network resources simultaneously.

As explained in this section, Section 7.2, DM inside the broker controls the $N$ compute and $Q$ network resources in the VPC, where $N$ and $Q$ change dynamically with user demand. Once requests have been scheduled, each new compute resource that is acquired by the system has the following characteristics:

Start time for the $j^{\text {th }}$ compute resource is denoted by start $j$ and is computed as -

\section{start $_{j}=\left(E S T\right.$ of the request expected to execute earliest on the $j^{\text {th }}$ compute resource $)-T$ seconds}

Note that compute resources are not made available immediately by the public cloud provider and the additional $T$ seconds is for handling the delay involved in starting a resource. Our observation with Amazon's EC2 was that that the service provider took 120 to 240 seconds to start a resource ( $\mathrm{t} 2$ type instances). Generally, researchers agree that provisioning compute resources may take up to 15 minutes [8].

Stop time for the $j^{\text {th }}$ compute resource $s t o p_{j}$ and is computed as -

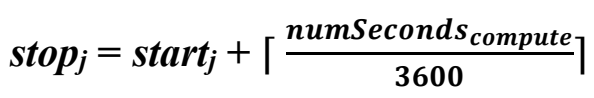


where numSecondscompute is the difference in seconds between the expected completion time of the last request on resource $j$ and start $_{j}$. Since a typical public cloud provider charges by the hour, the compute resource is held until the end of this "paid hour" period. Each resource $j$ has an internal timer which is set to expire at the time stop $_{j}$.

Additionally, after the requests have been scheduled, new network resources that are acquired by the system have the following characteristics:

Time at which the $k^{\text {th }}$ network resources is acquired is denoted by acquire $_{\boldsymbol{k}}$.

Before DM accepts the request, it reserves the different links to transfer the input data from the DS to the compute resource. The DM also reserves links to transfer the output data from the compute resource to one DS. Each link is reserved exactly at the time when data is required to be transferred.

Release time for the $k^{\text {th }}$ network resource is denoted by release $k$ and is computed as -

release $_{k}=$ acquire $_{k}+\left\lceil\frac{\text { numSeconds }}{\text { network }}\right\rceil$

where numSecondsnetwork is the difference in seconds between the expected completion time of the last data transfer on the $k^{\text {th }}$ network resource and acquire . $_{\text {. }}$

Acquiring and releasing a resource is based on the three following rules:

Rule I: When $B P_{i}>0$, acquire $j^{\text {th }}$ compute resource and $\mathrm{k}$ network resources

Rule II: When $\left(s t o p_{j}\right)=$ current time, release $j^{\text {th }}$ compute resource and $\mathrm{k}$ network resources

Rule III: If rejecting the request would violate a Grade of Service (GoS) criterion specified by the user, acquire $j^{\text {th }}$ compute resource and $\mathrm{k}$ network resources

Rule I states that the $j^{\text {th }}$ compute resource and $k$ network resources are acquired if a profit is generated for the broker. Rule II specifies the release of the $j^{\text {th }}$ compute resource and $k$ network resources at their stop time. Rule III states that even if the calculated value of $B P_{i} \leq 0$, 
DM needs to check whether the GoS criterion is satisfied. Although the system described in this research uses the blocking ratio for describing the GoS, the framework can be adapted to other metrics as well. The auto-scaling algorithm (presented in Table 7.1) is described next. The goal of the auto-scaling algorithm is to allocate the necessary resources for the request to complete before its specified deadline. To execute a request, the following steps need to be achieved:

1. Transfer input data from the DS to the resource where the request will be executed.

2. Execute the request.

3. Transfer the output data back from the resource to DS.

When a user sends a request, the broker needs to predetermine whether it would be profitable to accept the request, schedule the request by reserving the compute and network resources and inform the user about the cost. The broker controls a set of $N$ compute resources and a set of $Q$ network links with a corresponding set of $Q$ bandwidth reservations.

$\operatorname{Res}=\left\{\operatorname{Res}_{1}, \operatorname{Res}_{2}, \ldots \operatorname{Res}_{N}\right\}$ is a set of $N$ compute resources acquired by the broker Link $=\left\{\operatorname{Link}_{1}, \operatorname{Link}_{2}, \ldots, \operatorname{Link}_{Q}\right\}$ is a set of $Q$ links available to the user with bandwidths $B$ $=\left\{B_{1}, B_{2}, \ldots, B_{Q}\right\}$. The bandwidth reservation for the $Q$ links controlled by the broker.

Here are the input and output parameters for the algorithm.

Input Data: $R e q_{i}$ is the incoming request sent by $\mathrm{SCE}$ $D S I_{i}=\left\{D S I_{i l}, D S I_{i 2}, \ldots, D S I_{i p}\right\}$

$D S I_{i}$ is a set of input data stores from where data is to be transferred to the $j^{\text {th }}$ compute resource which will execute the $i^{\text {th }}$ request.

$D S O_{i}$ is the output data store for storing the result of the computation for the $i^{t h}$ request.

Output data: $\left\{\operatorname{Res}_{j},\left\{\operatorname{Link}_{k}, B_{k}\right\}\right\}$

$\operatorname{Res}_{j}$ is the resource selected for executing the $i^{\text {th }}$ request. 
$L_{i n k}$ is the set of links connecting Res $j$ with the input datastore DSIi and the output datastore $D S O i . B_{k}$ is the set of bandwidth requirements that are met when reserving the set of network links given by Link .

The auto-scaling algorithm is described in Table 7.1 After a request arrives at the broker, the broker loops through all compute resources that it controls to find a resource that can accommodate the request with the cheapest network cost. The costForRequest stores the cost for request $i$ to be executed on resource $j$. It then determines the lowest network cost from each of the $p$ DS resources using the findCheapestRoute sub-algorithm, which is based on Dijkstra's shortest path algorithm, which computes the lowest link cost based on the current network resources acquired by the broker. Since the cost of network links is the same, reducing the cost also reduces the number of network links used, thus reducing the East-West traffic. The computation of minimum cost for the request obtained by selecting the cheapest route between the data stores and the compute resource is shown from lines $3-5$. If the request $i$ cannot be scheduled on the resource $j$, the total cost is set to infinity. Finally, the cost to transfer the data back to DSF is calculated. This is shown in lines $6-10$. Lines $11-17$ determine whether scheduling the request on the selected resource leads to a profit for the broker. Line 12 uses a sub-algorithm computeUserCost for determining the cost the End User pays the broker for using the same resources being reserved by the broker, computed by replacing the broker costs with the user costs.

This algorithm loops through all the compute resources in order to determine the best fit for the request on the resource and selects the resource with the minimum cost for the user. If the request does not lead to a profit for the broker but the request needs to be accepted for meeting the GoS criterion, the broker acquires a new resource from the cloud as shown in line 19. Selection 
of compute resources work in a similar fashion to previous chapters, where the broker has no control over the physical location in the cloud data center where the compute resource is assigned from. Network resources are chosen by the broker directly.

Table 7.1 Auto-Scaling Algorithm with Network Resources

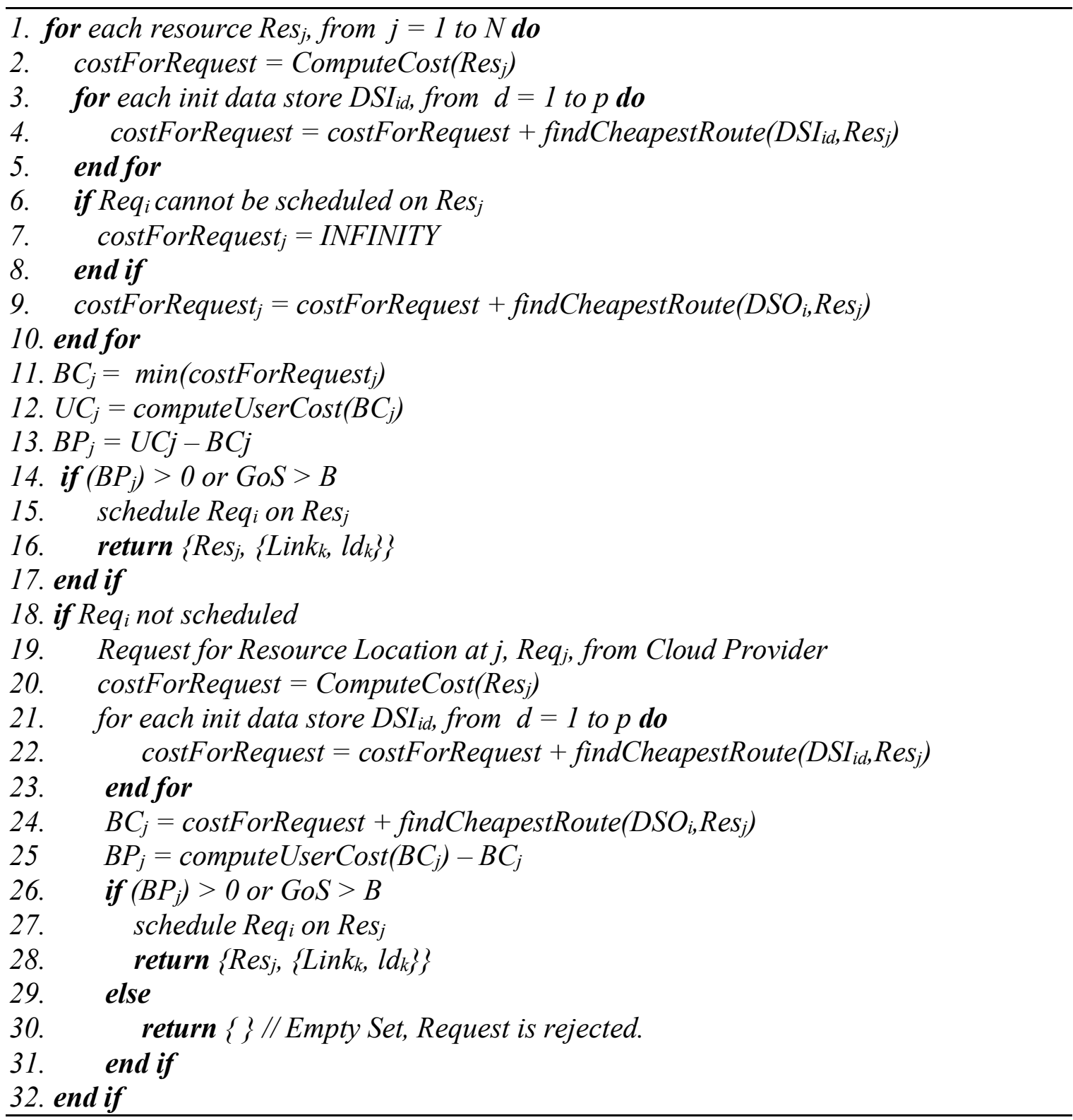

Once the broker has been assigned a compute resource and corresponding network resources, the broker tries to validate if the request accrues a profit for the broker or not and 
returns the resources with the selected network links or an empty set, signifying no compute nor network resources were reserved, and the request was rejected, as shown from lines $18-31$. As with previous approaches presented in chapters Chapter 4, Chapter 5 and Chapter 6, each resource is only acquired for the paid hour, and resources are released after the end of the paid hour.

\subsection{Performance Analysis}

A simulation-based analysis of the performance of the algorithm presented in Table 7.1 was performed. Each simulation experiment was sufficiently long to ensure that the system had reached steady state. An experiment was repeated a sufficient number of times to achieve an interval that is less than $\pm 5 \%$ for the performance metrics broker profit, user cost and DCN utilization described in Section 7.4.1.4 for a confidence level of 95\%. A description of the performance metrics, workload parameters, the data-center configuration and system parameters used in the simulation experiments are presented next. The results of the experiments are discussed in Section 7.5.

\subsubsection{Experimental Parameters}

This Section focuses on discussing the experimental parameters that are used in analyzing the performance of a simulation-based system that implements the proposed auto-scaling algorithm and compares its performance with that of another system from the literature [68]. The performance analysis presented in Section 7.5 includes a discussion on how changing parameter values affect the performance of the system. Each experiment is performed by changing one parameter at a time while holding the other parameters at their default values (see Table 7.2 and Table 7.3). Column 2 in each table specifies the set of all values used for the respective parameter. The default values described in the table are shown in boldface. The data center setup used for running the simulation is presented in 
Section 7.4.1.3. Section 7.4.1.1 presents the workload parameters whereas the system parameters are presented in Section 7.4.1.2.

\subsubsection{Workload Parameters}

The values for each parameter used are given in Table 7.2. Most parameters have been described in Section 4.4.1. The newer workload parameters involving the network and data resources are described next.

Mean number of Input Data Stores $\left(D S_{I}\right)$ - is $p$ number of data stores that the request needs data from before it can begin its execution. The number of $D S$ for a request is uniformly distributed between 1 and 5. Although the number depends on the characteristics of the workload, typical data-center application requests transfer data from up to 5 nodes [69]. The mean volume of total data transfer to all $D S$ per request is $500 \mathrm{~Gb}$, generated using an exponential distribution.

Table 7.2 Summary of Workload Parameters

\begin{tabular}{|l|l|}
\hline Load Factor: $\boldsymbol{f}$ & $0,0.2, \mathbf{0 . 4}, 0.6,0.8,1$ \\
\hline Mean Service Time (in mins): $\boldsymbol{S}$ & $\mathbf{5 0}$ \\
\hline Laxity Factor: $\boldsymbol{L f}$ & $\mathbf{2}$ \\
\hline Mean Arrival Rate (in reqs/sec): $\boldsymbol{\lambda}$ & $0.0083,0.0067, \mathbf{0 . 0 0 5 3}, 0.0037, \mathbf{0 . 0 0 2 7}$ \\
\hline Mean Number of Input n DS: $\boldsymbol{D} \boldsymbol{S}_{\boldsymbol{p}}$ & $1-5$, Uniform Distribution \\
\hline Number of Output DS: $\boldsymbol{D} \boldsymbol{S}_{\boldsymbol{o}}$ & 1 \\
\hline Mean Data Transferred init & $500 \mathrm{~Gb}$, Exponential Distribution \\
\hline Mean Data Transferred final & $50 \mathrm{~Gb}$, Exponential Distribution \\
\hline
\end{tabular}

Number of Output Data Stores $\left(D S_{O}\right)$ - is the number of data stores that the request transfers 
data to after completion of execution. Each request has $1 D S F$. The mean size for the data transferred to the $D S_{O}$ is $50 \mathrm{~Gb}$, also modelled using an exponential distribution.

\subsubsection{System Parameters}

As used in Chapter 4, Chapter 5 and Chapter 6, the default public cloud provider cost is 2 cents for one hour of using a compute instance. Other compute costs for system parameters are the same as described in Section 4.4.2. New system parameters are described next. The network cost for the broker is given is 0.5 cents per hour per Gbps, being charged by the public cloud provider to the broker. The speed of the routers is given for the routers, along with the total number of routers of a type used in a system. The system parameters are given in Table 7.3.

Table 7.3 Summary of System Parameters

\begin{tabular}{|l|l|}
\hline User Compute cost rate (in \$ per sec): $\boldsymbol{c} \_\boldsymbol{p} \boldsymbol{v} \boldsymbol{t}_{\text {compute }}$ & $\mathbf{0 . 0 0 0 0 0 8 3 3}$ \\
\hline Broker Compute cost rate (in \$ per hour) for a micro instance: & $\mathbf{0 . 0 2}$ \\
$\boldsymbol{c} \_$pub $\boldsymbol{b}_{\text {compute }}$ & \\
\hline Broker Network cost rate (in \$ per hour per 1 Gbps): $\boldsymbol{c} \_$pvt $\boldsymbol{t}_{\text {network }}$ & $\mathbf{0 . 0 0 5}$ \\
\hline User Network cost rate (in \$ per second per 1 gbps): $\boldsymbol{c} \_$pub $\boldsymbol{b}_{\text {network }}$ & $\mathbf{0 . 0 0 0 0 0 5 5}$ \\
\hline
\end{tabular}

\subsubsection{Data center Configuration}

The data center configuration used in the simulation for the experiments is given in Table 7.4. There is one core router, 5 aggregate routers and 20 Top of the rack routers. Each $\mathrm{AR}$ is connected to the $\mathrm{CR}$ with $5 \mathrm{Gbps}$ links. Each TOR is connected to some of the ARs, with link speeds of $10 \mathrm{Gbps}$. Each TOR has 5 hosts connected and each one data store. 
Table 7.4 Data Center Configuration

\begin{tabular}{|l|l|}
\hline Core Router (CR) & $\mathbf{1}$ \\
\hline Aggregate Routers (AR) & $\mathbf{5}$ \\
\hline TOR/Servers & $\mathbf{2 0}$ \\
\hline Compute Hosts & $\mathbf{1 0 0}$ \\
\hline Data Stores & $\mathbf{2 0}$ \\
\hline
\end{tabular}

\subsubsection{Performance Metrics}

The performance metrics $B P$ and $U C$ have been described previously in Section 4.4.3. However, this work also auto-scales network resources. One of the goals of this chapter is to reduce the East-West traffic, and DCN Utilization is used to measure the reduction in such traffic.

DCN Utilization $(D U)$ - measures the total utilization for all the links in DCN and is computed by taking the average number of DCN links used by the broker divided by the total number of links in the DCN multiplied by 100 .

A higher $B P$ and a lower $U C$ indicates good system performance. A lower $D U$ indicates better DCN performance.

\subsubsection{Alternate Systems}

The system described in Section 3.3 also referred to as System I-RN is compared with other systems. A simulation of this system is created to aid in the comparison, instead of a prototype, since most cloud providers do not support auto-scaling network resources yet. This system is compared with an alternative system which is discussed in the previous chapters [68]. This system is referred to as System II-RN. System II-RN has much of the 
same properties as System II discussed in Section 3.3 but was discussed in context with compute resources. We extend the work to acquire and release network resources in a similar manner so as to compare with System I-RN. In System III-RN, users get the resources, both network and compute, directly from the public cloud provider without going through any broker (in the intermediary enterprise). As in the previous chapters, System III-RN, only total user cost is compared to that of System I-RN and System II-RN because there is no broker involved with System III-RN and hence no profit to be accrued for that system.

\subsection{Experimental Results}

Simulation experiments were performed to analyze the effect of various system and workload parameters on the efficacy of the auto-scaling technique described in the previous sections. The results of experiments provided in the following subsections demonstrate the extendibility of the auto-scaling approach described for handling compute resources in a cloud (discussed in the earlier chapters) to the autoscaling of both compute and network resources.

\subsubsection{Impact of Arrival Rate}

A performance analysis of a system subjected to a single arrival rate is performed to investigate the impact of $\lambda$ on profit. Figure 7.3 , presents the effect of $\lambda$ on the profit generated on System I-RN and System II-RN. BP in both systems is directly proportional to the arrival rate. This is because at higher arrival rates, the system receives a higher number of requests per unit time, thus increasing the potential for earning a higher profit/hour. System I-RN accrues up to $69 \%$ to $98 \%$ more profit earned by System II-RN. So far, the broker profit has considered the total profit earned by leasing both the compute 
and network resources. Figure 7.4 shows a breakdown of the profit earned by each system by network and compute resources. The broker in System I-RN earns $81 \%$ to $111 \%$ higher than the profit earned by the broker in System II-RN in terms of compute cost. The broker in System I-RN also earns $64 \%$ to $93 \% \%$ higher than the profit earned by the broker in System II-RN in terms of network cost. However, for any given arrival rate, the total amount of network profit seems to be higher than the compute profit, since the pricing for the two resources are different and network resources cost more to lease per hour.

\subsubsection{Impact of Load Factor}

Figure 7.5, compares the profit that accrues on System I-RN and System II-RN for different values of $f$. For all the values of $\mathrm{f}$ presented in the figure, System I-RN can generate more profit than System II-RN. The profit is inversely proportional to $f$ : as $f$ increases, profit decreases. For example, when $f$ is $0, B P$ is the highest. Note that at $f=0$, the system is subjected to only the higher arrival rate. As $f$ increases, the proportion of time for which the system is subjected to the lower arrival rate increases. At $f=1$ the system is subjected to only the lower arrival rate and the profits earned are lower, since a lower number of requests per hour reduces the potential of the broker to accrue a higher profit.

Overall, for a given $f, B P$ for System I-RN is up to $99 \%$ higher than System II-RN (see Figure 7.5). Figure 7.6 presents a comparison of the total user cost incurred in all the three systems. As shown in the figure, System I-RN leads to a lower total user cost in comparison to the other two systems. System I-RN provides cost savings up to $74 \%$ and $80 \%$ in comparison with System II-RN and System III-RN respectively. All requests are considered to be arriving from a group of users that belong to the client enterprise, abstracted as the user module in the simulation experiments. As a result, an increase in 
arrival rate implies a higher number of user requests per unit time resulting in a higher cost per hour for the user.

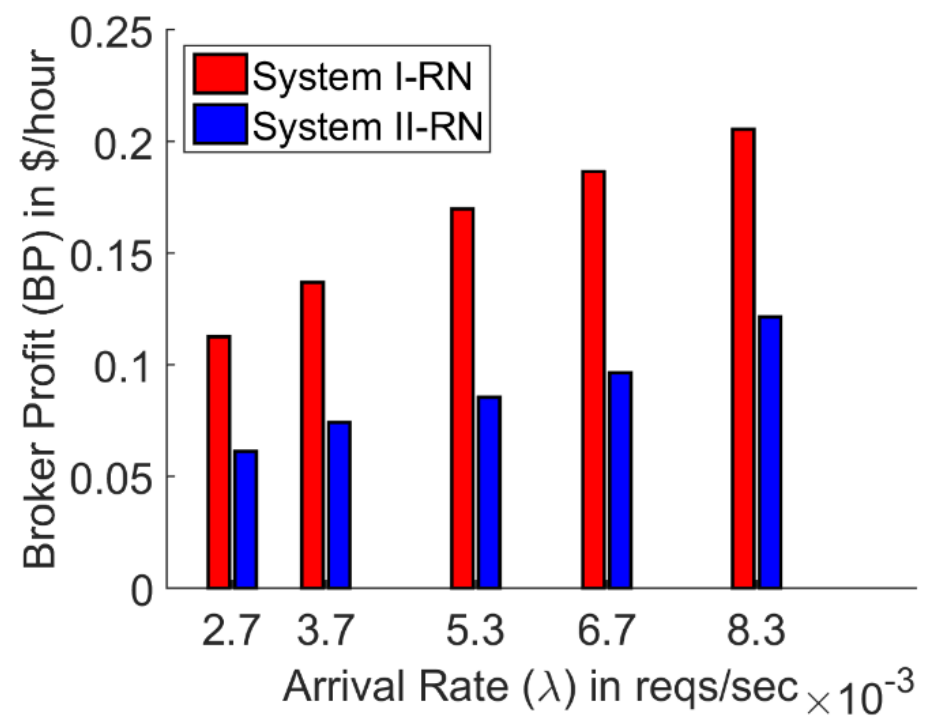

Figure 7.3. Impact of $\lambda$ on BP

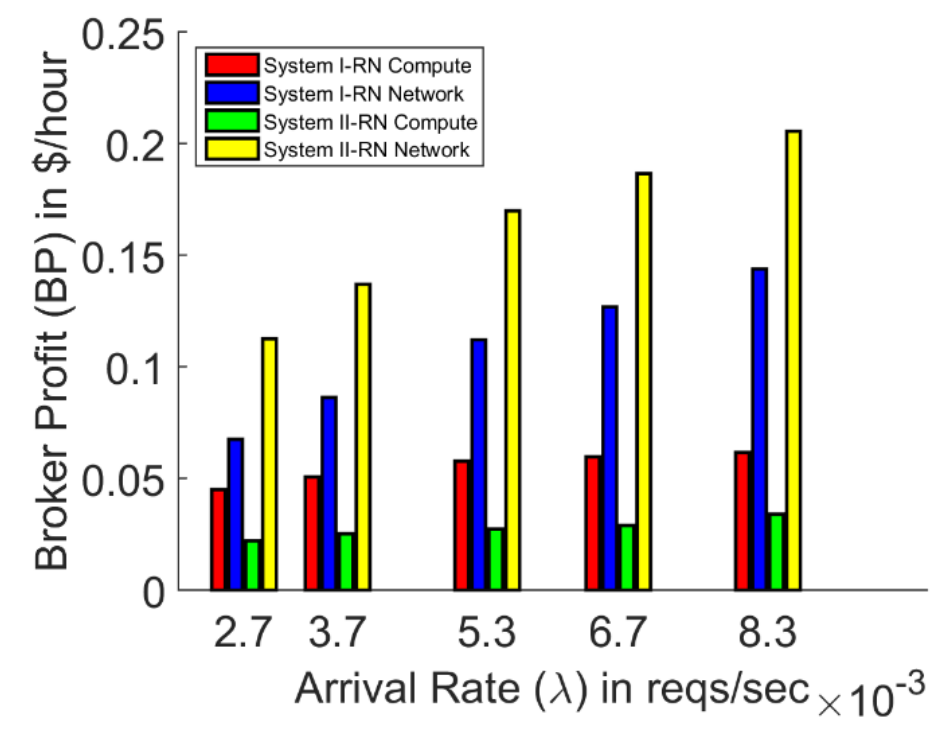

Figure 7.4. Impact of $\lambda$ on $B P$ by Resource

\subsubsection{Measure of East-West Traffic}

In Figure 7.7, the effect of changing the matchmaking algorithm to select compute and 
network resources is studied to observe the impact of East-West traffic generated. The goal of this work is to reduce the East-West traffic and in turn, reduce the utilization of the DCN. All the parameter values are kept at their default values. The default matchmaking algorithm used in this paper is the best fit matchmaking algorithm.

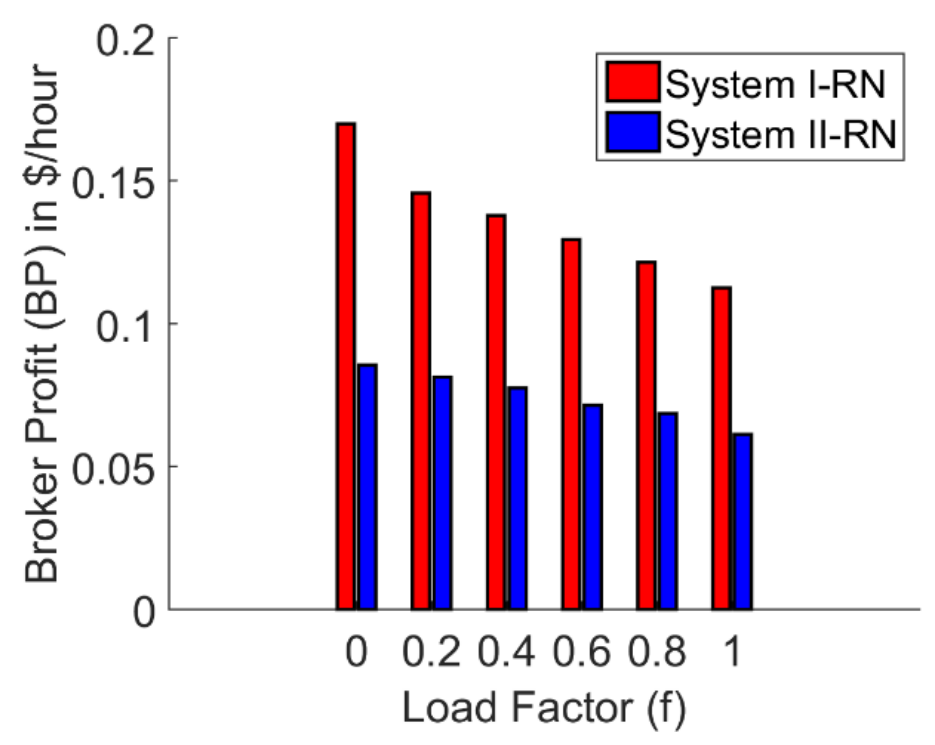

Figure 7.5. Impact of $f$ on Broker Profit

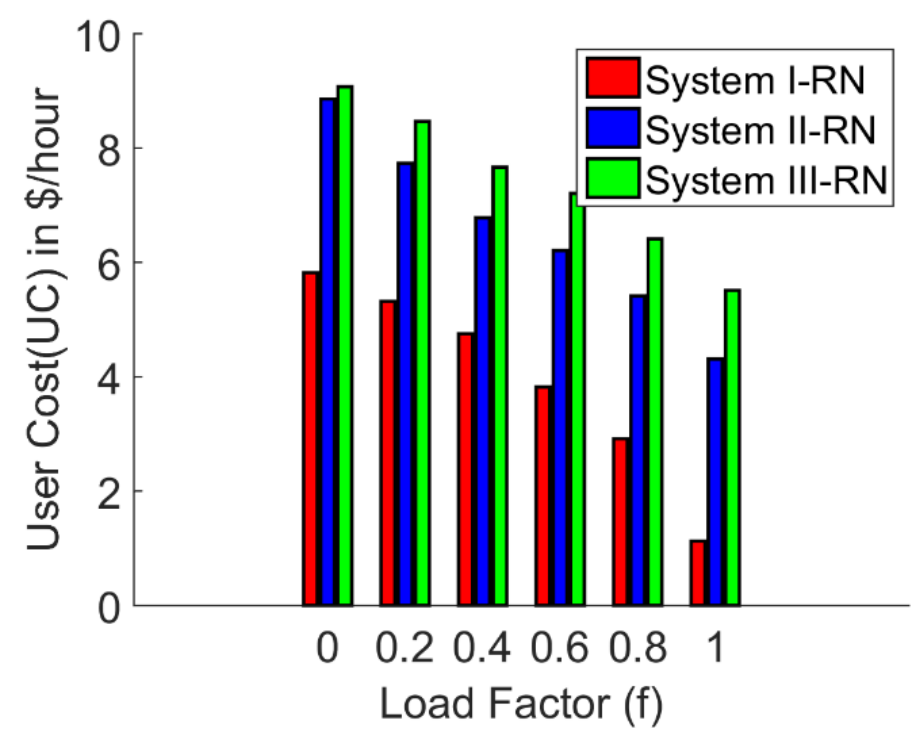

Figure 7.6. Impact of $f$ on User Cost

The performance of the best fit strategy is compared to that of first fit and best fit 
strategies. The best fit strategy selects the compute resource that reduces the number of links used to transfer all data during a request. The first fit strategy chooses the first viable compute resource that is able to meet the SLAs of the request. As shown in Figure 7.7, the best fit matchmaking algorithm uses the fewest links in the DCN. However, it incurs more overhead in comparison to the first fit, because it computes the shortest path and hence is more time consuming to compute than the first fit matchmaking strategy. The worst fit strategy uses the longest path and the greatest number of links. As expected, the worst fit approach performs the worst as shown in Figure 7.7.

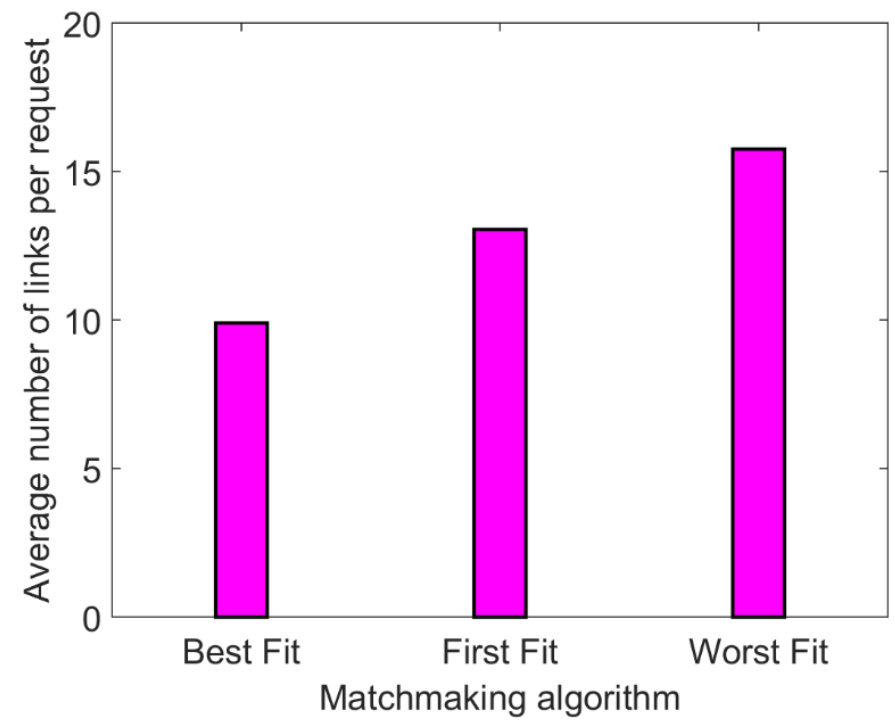

Figure 7.7. Comparison of Match-making Algorithms

The effect of increasing the number of data sources for each request on DU is presented in Figure 7.8. It is observed that as the number of data sources increases, the average DCN becomes saturated and the utilization for DCN reaches close to $100 \%$. However, for any given value of the number of data sources lower than 50, using the best fit algorithm results in a lower $D U$. The average $D U$ achieved by best fit is observed to be up to $60 \%$ lower than that achieved by first fit and up to $150 \%$ lower than that achieved by worst fit. This is 
because the best fit algorithm uses the fewest number of links to transfer data specified in the TAG requirements. Although this work does not support placement of data stores, the best-fit algorithm chooses the compute resource with the fewest number of links to the majority of the data sources for a given request. This demonstrates the reduction in EastWest network traffic achieved by the proposed technique that uses the best fit algorithm in comparison to a system that is based on the first fit technique for matchmaking.

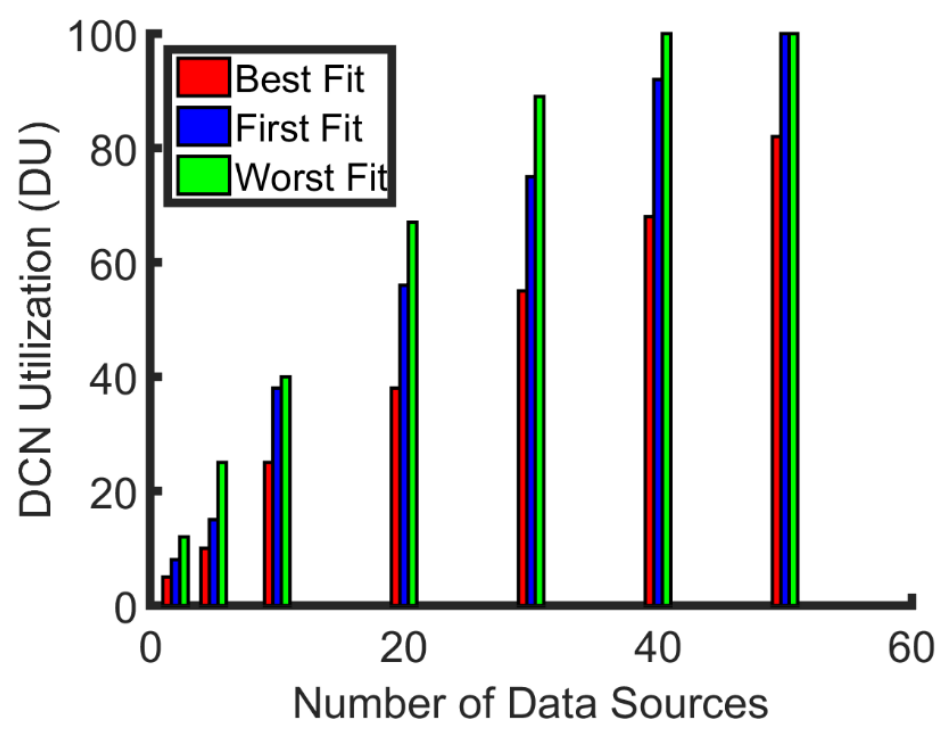

Figure 7.8. Impact of Changing the Number of Data Sources

\subsection{Experimental Observations}

This chapter focuses on auto-scaling of compute and network resources on clouds. It describes a novel algorithm and framework for auto-scaling performed by the broker that provisions compute and network resources from a public cloud on-demand. The proposed technique automatically determines the number of compute and network resources to acquire based on the profit earned by an intermediary enterprise while also monitoring the system state to ensure the system maintains a minimum grade of service. The system also reduces the amount of East-West traffic in the DCN by placing compute requests close to 
the data-stores in order to minimize network requests. As demonstrated through simulation experiments, the proposed technique can generate a profit for the intermediary enterprise hosting the broker while reducing the cost incurred by users, for the cost parameters chosen in this work. The framework is capable of handling both AR and OD requests. A number of important features of the technique are summarized:

- The work presented in this chapter demonstrates the ability of the reactive auto-scaling approach to handle both network and computing resources: the number of compute and network resources in the pool used by the user requests need not be determined a priori and can be controlled dynamically thereby reducing the cost for capacity planning. The additional mechanisms introduced for handling network resources in the algorithm described in Table 7.1 can be easily adopted for the proactive and hybrid auto-scaling algorithms. It is thus expected that the reactive, proactive and hybrid approaches discussed in Chapter 4, Chapter 5 and Chapter 6 respectively can be extended to handle network resources in addition to compute resources. A detailed investigation forms an important direction for future work.

- East-west traffic is reduced by placing compute jobs near the data storage locations.

- The auto-scaling technique leverages network virtualization to create multiple virtual networks (VNs) on top of a shared physical network enabling bandwidth sharing of individual links belonging to a DCN.

- The framework allows users in an enterprise for example, to submit AR as well as OD requests to a private cloud provided by the intermediary cloud provider where resources from a public cloud are used to handle the workload.

- The framework provides a minimum grade of service to ensure that a certain percentage 
of user requests are guaranteed to be executed.

A number of insights into system behaviour and performance obtained for the performance analysis are presented next.

- The experimental results demonstrate that using the proposed broker can lead to a higher profit as compared to other auto-scaling systems. For example, as shown in Figure 7.3, an improvement in $B P$ up to $98 \%$ has been observed. The broker profit is observed to depend on parameters, such as load factor, arrival rate, mean service time, laxity factor and user cost.

- A higher mean arrival rate enables the broker to earn a higher profit. As a result, lower the load factor higher is the difference between the profit generated by the proposed system and that generated by a conventional system.

- The broker profit is directly proportional to the user charge incurred by the client enterprise and to the mean service time of the request.

- For all the experiments performed, and for all the given parameters, the total network profit is observed to be higher than the compute profit.

- The techniques described in this chapter reduces the East-West traffic and in turn, reduces the utilization of the DCN by employing a best fit matchmaking algorithm. However, it incurs additional computational overhead in comparison to systems using the first fit matchmaking algorithm. The additional overhead did not lead to a significant increase in the proportion of jobs rejected in the experiments described in the chapter. 


\section{Chapter 8 Summary and Conclusions}

This chapter concludes the thesis. Section 8.1 summarizes the proposed techniques and algorithms. An alternative pricing model is discussed in Section 8.2 that explains how the work presented in this thesis may be useful in the context of changes to the pricing structure that may be made by cloud providers. Finally, Section 8.3 provides a closing discussion with insights into system behavior and performance based on the experimental results and presents a list of topics that can form directions for future research.

\subsection{Summary}

This research focuses on auto-scaling resources in a data center. Auto-scaling is a technique where the amount of resources utilized in supporting request execution in a cloud environment, changes automatically based on system load. Most of the chapters focus on auto-scaling only compute resources. Chapter 7 discussed the capability of the proposed auto-scaling techniques to handle network resources in addition to compute resources. The thesis introduces a broker that is operated by an organization referred to as the Intermediary Enterprise. The broker auto-scales the number of resources acquired from a cloud provider based on the workload demand from requests sent by an organization known as the Single Client Enterprise.

This research handles Service Level Agreements for the workload by allowing requests to specify an earliest start time and deadline for the requests. These types of requests are also referred to as advance reservation requests. The research also handles requests without an SLA requirement. These requests are known as on-demand requests and have no deadline associated with them.

SCE sends requests to IE on behalf of these users. IE is charged by the public cloud 
provider to rent resources, and IE in turn charges SCE. The broker auto-scales the resources in order to increase the profit earned from its operations while attempting to reduce the cost incurred by SCE. A request may be rejected by the broker if the request does not accrue a profit for the broker. The research also introduces a grade of service that ensures that a minimum proportion of requests, specified by the SCE, is always accepted by the broker in order to maintain a desired Quality of Service. Thus, the broker maintains a specified GoS set by SCE, thereby providing SCE with a service level GoS guarantee. A high-level overview of the research contributions is presented next.

- Three novel auto-scaling algorithms have been devised that make use a price model that leads to an increase in the profit for the broker (intermediary enterprise) and a reduction in the user cost at the same time. These are briefly described.

$\circ$ A reactive auto-scaling algorithm that changes the resources for the SCE in response to a change in the system workload is proposed in Chapter 4. This algorithm is executed on each request arrival and auto-scaling is based on the demands specified by the request and the current system load.

- A proactive auto-scaling algorithm that uses past workload for the system to predict the characteristics of the requests that are expected in the future. The proactive algorithm, which uses this forecast to decide on the number of resources to be deployed when these predicted requests arrive, is described in Chapter 5. This algorithm is executed periodically. When new requests arrive, they are scheduled on the available resources.

- A hybrid auto-scaling algorithm that combines the advantages of both reactive and proactive techniques and is described in Chapter 6. This technique acquires 
resources before request arrivals to ensure most arriving requests have resources to be executed on. This technique also allows for accommodating errors in the prediction by scaling up reactively for incoming requests, if resources are unavailable.

- A detailed performance analysis of the algorithms using a prototype implementation as well as a simulation is undertaken. The analysis provides insights into broker profit and user cost for various combinations of system and workload parameters.

- A reactive auto-scaling framework discussed in Chapter 7 allows network resources to be handled in addition to compute resources. This increases the capability of the autoscaling frameworks to handle different resources.

- The auto-scaling technique introduced in Chapter 7 also helps reduce the East-West traffic in the Data Center. This, in turn, reduces the utilization of the Data Center Network.

\subsection{Using Alternate Resource Pricing}

The thesis research that started in 2013 is based on a pricing scheme in which the public cloud provider charges the consumer on a per hour basis. Incurring an hourly charge was the only way an on-demand instance could be rented by the consumer at that time. This is still the practice today for renting instances running enterprise-level operating systems such as Microsoft Windows, SUSE Linux Enterprise Server and Red Hat Enterprise Linux instances, where users pay an hourly charge for renting instances running those OS types. More recently (after September 2017), some cloud infrastructure providers such as Amazon and Microsoft have started offering compute instances with non-enterprise OS licenses, typically with a Linux OS that is free for non-commercial use, that may be 
rented on a per second basis by a service consumer [70]. Although the same techniques described for broker scheduling for handling the SLAs associated with client requests, as well as maintaining a GoS for the application can be used in order to generate a profit for IE, a different type of pricing scheme needs to be used for the broker to rent instances from the public cloud provider.

Another instance type with an alternate pricing scheme is popular among most cloud providers. These instance types are known as Reserved Instances and have been mentioned in Section 1.1.4.2. A Reserved Instance leads to a reservation of resources and capacity, for either one or three years [71]. Unlike on-demand instances that require users to only pay for the hours (or seconds) used, users renting reserved instances must commit to paying for the entire 1 or 3 year terms. In exchange, the hourly rate is lowered significantly by the cloud provider.

Furthermore, the cloud provider typically offers a discounted price when a user rents a reserved instance in comparison to on-demand prices. For example, Amazon offers a significant discount (up to 75\%) compared to On-Demand instance pricing [72]. Although most cloud providers only offer a 1 year or a 3 year leasing term, Amazon has a marketplace where users rent reserved instances for a much shorter period. Hence, it is possible to lease reserved instances for a month, by still paying lower than acquiring the same instance with on-demand pricing.

This research can use reserved instances in a similar manner as on-demand instances by renting reserved instances when required. However, the drawback with adopting this approach is forcing the broker to keep paying for the infrastructure even though the resources may remain unutilized. A conservative estimate of the number of resources 
required to handle the workload needs to be made. The broker must acquire only a portion of the total number of reserved instances that are deemed to be required for handling the workload. For example, if the broker determines that the number of instances required for handling the peak workload is 10 for the next month, it can suggest renting $70 \%$ of the required capacity, or 7 resources. If an increase in the workload at a given point in time requires additional resources for maintaining the GoS, on demand instances can be acquired by the broker for satisfying these requests. Although these on-demand resources (rented on a per second basis), will not lead to any broker profit and overall broker profit will still be accrued from the use of cheaper reserved instances.

\subsection{Conclusions}

A proactive, a reactive and a hybrid approach for auto-scaling were proposed. The three auto-scaling approaches introduced in this thesis were analyzed through simulation and prototyping and measurement. The results of this analysis demonstrate the effectiveness of these techniques.

A detailed discussion of the performance of the three techniques including the impact of various workload and system parameters on performance are presented in Chapter 4, Chapter 5 and Chapter 6. A number of key insights resulting from the performance analysis are presented.

- For a range of system and workload parameters experimented with the three techniques gave rise to an improvement in both user cost and broker profit in comparison to a conventional system.

- A threshold value for the execution times for requests seems to exist. In order for the broker to remain profitable the execution time for request must 
be higher than this threshold value.

- The hybrid auto-scaling strategy effectively combines the advantages of both reactive and proactive auto-scaling. In most cases investigated (see Chapter 6) the hybrid auto-scaling technique seems to demonstrate superior performance in comparison to the pure reactive and proactive auto-scaling techniques.

- Three components of system overhead have been measured and discussed in Chapter 4, Chapter 5 and Chapter 6:

○ Internal Communications overheads.

- External Communications overheads.

○ Machine Learning Algorithm overheads.

The total overhead seems to be a very small proportion of the experimental run time. The maximum overhead is measured to be small.

- The thesis has focused primarily on the auto-scaling of compute resources. As discussed in Chapter 7, the auto-scaling techniques can be extended to include the auto-scaling of network resources as well.

- By reserving compute resources in close proximity to storage resources, this thesis managed to show a reduction of the East-West traffic in the DCN.

\subsubsection{Future Work}

Directions for future research include the following:

- This research is based on a synthetic workload. Evaluation of the proposed auto-scaling techniques for real-workload traces forms an interesting direction for future research. 
- As previously indicated in Section 7.6, a detailed investigation of the extension of the proactive and hybrid auto-scaling techniques to handle network resources is important.

- The computing resources used in this thesis are assumed to be identical to one another. Handling heterogeneous systems comprising of multiple types of computing resources warrants investigation.

- The auto-scaling techniques also need to be adapted with other system resources that include main memory and secondary disk storage.

- The proactive auto-scaling technique introduced in this research needs a training period during which no profit is incurred by the broker. Running a reactive auto-scaler concurrently during the training phase may enable the broker to earn a profit during this training phase. Such a system warrants further investigation.

- The auto-scaling approaches discussed in this thesis use only horizontal auto-scaling techniques in which the number of resources is increased or decreased for handling changes in the workload. A combination of horizontal scaling with vertical scaling of compute resources needs further investigation.

- Although the broker was not observed to be a performance bottleneck for the combination of system and workload parameters experimented with, such bottlenecks may occur on systems subjected to a higher workload intensity. Techniques for improving the scalability of the broker on such systems through vertical scaling and migrating the broker to a large CPU instance in response to an increase in workload intensity need investigation. 
The broker may also be re-engineered to use multiple compute instances to speed up its computations thus alleviating any performance bottleneck.

- Estimates of job run times provided by a user submitting an AR can be inaccurate. Previous work in [50] has described techniques for handling such inaccuracies in the context of grids. Adaptation of these techniques to auto-scaling on clouds forms a direction for future research. 


\section{References}

[1] M. Armbrust, A. Fox, R. Griffith, A. D. Joseph, R. Katz, A. Konwinski, G. Lee, D. Patterson, A. Rabkin, I. Stoica and M. Zaharia, "A View of Cloud Computing," Communications of the ACM, vol. 53, no. 4, pp. 50-58, 04, 2010.

[2] Amazon, "Amazon EC2 Pricing," [Online]. Available: http://aws.amazon.com/ec2/pricing/. [Accessed 07 2019].

[3] D. Gmach, J. Rolia, L. Cherkasova and A. Kemper, "Workload Analysis and Demand Prediction of Enterprise Data Center Applications," in Proc. of the 10th International Symposium on Workload Characterization, Boston, U.S.A, 09, 2007.

[4] C. Pemberton, "Hidden Cloud Growth Opportunities for Technology Service Providers," [Online]. Available: https://www.gartner.com/smarterwithgartner/7hidden-cloud-growth-opportunities-for-technology-service-providers/. [Accessed 07 2019].

[5] M. J. Turner and G. Tim, "Critical Application And Business KPIs For Successful Cloud Migration (White Paper)," AppDynamics, 08, 2017.

[6] P. Mell and T. Grance, "The NIST definition of cloud computing," NIST, Gaithersburg, U.S.A, 2011.

[7] S. Lehrig, H. Eikerling and S. Becker, "Scalability, Elasticity, and Efficiency in Cloud Computing: a Systematic Literature Review of Definitions and Metrics," in Proc. of the 11th International ACM Conference on Quality of Software 
Architectures (SIGSOFT), New York, U.S.A, pp. 83-92, 05. 2015.

[8] T. Lorido-Botran, J. Miguel-Alonso and J. A. Lozano, "Auto-scaling Techniques for Elastic Applications in Cloud Environments," EHU-KAT-IK-09-12, Madrid, Spain, 2012.

[9] I. Foster, C. Kesselman, C. Lee, B. Lindell, C. Nahrstedt and A. Roy, "A distributed resource management architecture that supports advance reservations and co-allocation," in Proc. of Seventh International Workshop on Quality of Service (IWQoS), London, U.K., pp. 27 - 36, 05, 1999.

[10] R. Buyya, S. K. Garg and R. N. Calheiros, "SLA-Oriented Resource Provisioning for Cloud Computing: Challenges, Architecture, and Solutions," in Proc. of the International Conference on Cloud and Service Computing (CSC), Hong Kong, China, pp. 1 - 10, 12, 2011.

[11] B. Kepes, "Understanding the Cloud Computing Stack: SaaS, PaaS, IaaS," [Online]. Available: https://support.rackspace.com/how-to/understanding-thecloud-computing-stack-saas-paas-iaas/. [Accessed 07 2019].

[12] "Microsoft Azure," Microsoft, [Online]. Available: https://azure.microsoft.com/en-us/. [Accessed 07 2019].

[13] S. Shakkottai, R. Srikant, A. Ozdagla and D. Acemoglu, "The Price of Simplicity," IEEE Journal on Selected Areas in Communications, vol. 26, no. 7, pp. 1269$1276,09,2008$.

[14] H. Xu and B. Li, "Dynamic Cloud Pricing for Revenue Maximization," IEEE Transactions on Cloud Computing, vol. 1, no. 2, pp. 2168-7161, 11, 2013. 
[15] Cisco, "Cisco Global Cloud Index: Forecast and Methodology, 2014-2019," Cisco, 2015.

[16] OpenStack Consortium, "Openstack," [Online]. Available: https://www.openstack.org. [Accessed 07 2019].

[17] Amazon, "Amazon Web Services," [Online]. Available: https://aws.amazon.com/. [Accessed 07 2019].

[18] Openstack Org., "Neutron's documentation," [Online]. Available: https://docs.openstack.org/neutron/latest/. [Accessed 2206 2019].

[19] H. Xu and B. Li, "Anchor: A Versatile and Efficient Framework for Resource Management in the Cloud," IEEE Transactions on Parallel and Distributed Systems, vol. 24, no. 6, pp. 1066 - 1076, 10, 2012.

[20] D. Milojicic, I. M. Llorente and R. S. Montero, "OpenNebula: A Cloud Management Tool," IEEE Internet Computing Archive, vol. 15, no. 2, pp. 11-14, 03, 2011.

[21] B. Sotomayor, R. S. Montero, I. M. Llorente and I. Foster, "Virtual Infrastructure Management in Private and Hybrid Clouds," IEEE Computer Society, vol. 13, no. 5, pp. 14 - 22, 09, 2009.

[22] W. Wang, D. Niuł, B. Li and B. Liang, "Dynamic Cloud Resource Reservation via Cloud Brokerage," in Proc. of 33rd International Conference on Distributed Computing Systems (ICDCS), Philadelphia, U.S.A, pp. 400 - 409, 07, 2013.

[23] Amazon, "Amazon CloudWatch," [Online]. Available: http://aws.amazon.com/cloudwatch/. [Accessed 07 2019]. 
[24] X. Dutreilh, N. Rivierre, A. Moreau, J. Malenfan and I. Truck, "From Data Center Resource Allocation to Control Theory and Back," in Proc. of 3rd IEEE Conference on Cloud Computing (CLOUD), Miami,, U.S.A, pp. 410-417, 07, 2010.

[25] RightScale, "RightScale Cloud Management.," [Online]. Available: https://www.rightscale.com/about. [Accessed 07 2019].

[26] T. C. Chieu, A. Mohindra, A. A. Karve and A. Segal, "Dynamic Scaling of Web Applications in a Virtualized Cloud Computing Environment," in Proc. of IEEE International Conference on e-Business Engineering (ICEBE), Macau, China, pp. $281-286,10,2009$.

[27] H. C. Lim, S. Babu, J. S. Chase and S. S. Parekh, "Automated control in cloud computing: challenges and opportunities," in Proc. of 1st workshop on Automated control for datacenters and clouds, New York, U.S.A, pp. 13-18 , 06, 2009.

[28] Y. Kouki and T. Ledoux, "SCAling: SLA-driven cloud auto-scaling," in Proc. of the 28th Annual ACM Symposium on Applied Computing, New York, U.S.A, pp. 411-414, 03, 2013.

[29] J. O. Melendez, A. Biswas, S. Majumdar, B. Nandy, M. Zaman, P. Srivastava and N. Goel, "A Framework for Automatic Resource Provisioning for Private Clouds," in Proc. of the 13th IEEE/ACM International Symposium on Cluster, Cloud and Grid Computing (CCGrid), Delft, Netherlands, pp. 610 - 617, 05, 2013.

[30] J. H. Novak, S. K. Kasera and R. Stutsman, "Cloud Functions for Fast and Robust Resource Auto-Scaling," in Proc. of 11th International Conference on 
Communication Systems \& Networks (COMSNETS), Bengaluru, India, pp. 133140, 01, 2019.

[31] L. R. Moore, K. Bean and T. Ellahi, "Transforming reactive auto-scaling into proactive auto-scaling," in Proc. of the 3rd International Workshop on Cloud Data and Platforms (CloudDP), New York, U.S.A, pp 7-12, 04, 2013.

[32] T. Lorido-Botran, J. Miguel-Alonso and J. A. Lozano, "A Review of Auto-scaling Techniques for Elastic Applications in Cloud Environments," Journal of Grid Computing, vol. 4, no. 12, pp. 559-592, 12, 2014.

[33] S. Bhagavathiperumal and M. Goyal, "Dynamic Provisioning of Cloud Resources Based on Workload Prediction," Lecture Notes in Networks and Systems (Springer), vol. 75, no. 1, pp. 41-49, 2019.

[34] A. Y. Nikravesh, S. A. Ajila and C.-H. Lung, "Towards an autonomic auto-scaling prediction system for cloud resource provisioning," in Proc. of the 10th International Symposium on Software Engineering for Adaptive and SelfManaging Systems, Florence, Italy, pp. 35-45, 05, 2015.

[35] Z. Gong, X. Gu and J. Wilkes, "PRESS: PRedictive Elastic ReSource Scaling for cloud systems," in Proc. of International Conference on Network and Service Management, Niagara Falls, Canada, pp. 9 - 16, 10. 2010.

[36] W. Fang, Z. Lu, J. Wu and Z. Cao, "PPS: A Novel Resource Prediction and Provisioning Scheme in Cloud Data Center," in Proc. of Ninth International Conference on Services Computing (SCC), Honolulu, U.S.A, pp. 609 - 616, 06, 2012. 
[37] L. R. Moore, K. Bean and T. Ellahi, "A Coordinated Reactive and Predictive Approach to Cloud Elasticity," in Proc. of Fourth International Conference on Cloud Computing, GRIDs, and Virtualization, Valencia, Spain, pp. 87 - 92, 05, 2013.

[38] B. Urgaonkar, P. Shenoy, A. Chandra, P. Goyal and T. Wood, "Agile dynamic provisioning of multi-tier Internet applications," ACM Transactions on Autonomous and Adaptive Systems (TAAS), vol. 3, no. 1, p. 39, 03, 2008.

[39] W. Iqbal, M. N. Dailey, D. Carrera and P. Janecek, "Adaptive resource provisioning for read intensive multi-tier applications in the cloud," Future Generation Computer Systems, vol. 27, no. 6, pp. 871-879, 06, 2011.

[40] S. Jain, "B4: Experience with a globally-deployed software defined wan," in in Proc. of the ACM SIGCOMM 2013 Conference on SIGCOMM'13, ACM, New York, U.S.A, pp. 3-14, 05, 2013.

[41] S. Rahman, T. Ahmed, M. Huynh, M. Tornatore and B. Mukherjee, "Auto-Scaling VNFs Using Machine Learning to Improve QoS and Reduce Cost," in Proc. of International Conference on Communications (ICC), Kansas City, MO, USA, pp. $1-6,05,2018$.

[42] C. Guo, G. Lu, H. J. Wang, S. Yang, C. Kong, P. Sun, W. Wu and Y. Zhang, "SecondNet: a data center network virtualization architecture with bandwidth guarantees," in Proc. of the 6th International Conference Co-NEXT, Philadelphia,, U.S.A, pp. 1 - 12, 12, 2010.

[43] M. Z. Hasan, E. Magana, A. Clemm and S. L. D. Gudreddi, "Integrated and 
autonomic cloud resource scaling," in Proc. of Network Operations and Management Symposium (NOMS), Maui,, U.S.A, pp. 1327 - 1334, 04, 2012.

[44] M. Dalton, "Andromeda: Performance, Isolation, and Velocity at Scale," in Proc. of 15th \{USENIX\} Symposium on Networked Systems Design and Implementation, Renton, U.S.A, pp. 373-387, 04, 2018.

[45] Amazon, "Amazon VPC," [Online]. Available: http://aws.amazon.com/vpc/. [Accessed 07 2019].

[46] M. Mazzucco and M. Dumas, "Reserved or On-Demand Instances? A Revenue Maximization Model for Cloud Providers," in Proc. of IEEE International Conference on Cloud Computing (CLOUD), Washington, U.S.A, pp. 428 - 435, 07, 2011.

[47] J. O. Melendez and S. Majumdar, "Matchmaking on Clouds and Grids," Journal of Internet Technology, vol. 13, no. 6, pp. 853-866, 11, 2012.

[48] H. Wang, J. Jin, Z. Wang and L. Shu, "On a novel property of the earliest deadline first algorithm," in Proc. of Eighth International Conference on Fuzzy Systems and Knowledge Discovery, Shanghai, China, pp. 197 - 201, 07, 2011.

[49] N. Lim, S. Majumdar and P. Ashwood-Smith, "MRCP-RM: A Technique for Resource Allocation and Scheduling of MapReduce Jobs with Deadlines," IEEE Transactions on Parallel and Distributed Systems, vol. 28, no. 5, p. 14, 2017.

[50] U. Farooq, S. Majumdar and E. W. Parsons, "Achieving efficiency, quality of service and robustness in multi-organizational Grids," Journal of Systems and Software, vol. 82, no. 1, p. 15, 2009. 
[51] J. Yu, R. Buyya and C. K. Tham, "Cost-based scheduling of scientific workflow applications on utility grids," in Proc. of the First International Conference on eScience and Grid Computing, Melbourne, Australia, pp. 140 - 147, 07, 2005.

[52] R. T. Fielding and R. N. Taylor, "Principled design of the modern Web architecture," in Proc. of the 22nd international conference on Software engineering, New York, U.S.A, pp. 407- 416, 06, 2000.

[53] Pivotal Software, "Spring," [Online]. Available: https://spring.io/. [Accessed 07 2019].

[54] Gradle Inc., "Gradle," [Online]. Available: http://gradle.org/. [Accessed 07 2019].

[55] The Apache Software Foundation, "Maven," [Online]. Available: https://maven.apache.org/. [Accessed 07 2019].

[56] A. Sulistio and R. Buyya, "A Grid Simulation Infrastructure Supporting Advance Reservation," in Proc. of the 16th International Conference on Parallel and Distributed Computing and Systems, Boston, U.S.A, pp. 1 - 7, 11, 2004.

[57] J. Sim and N. Reid, "Statistical Inference by Confidence Intervals: Issues of Interpretation and Utilization," Physical Therapy, vol. 79, no. 2, p. 186-195, 02, 99.

[58] G. Box, G. Jenkins, G. Reinsel and G. Ljung, Time Series Analysis: Forecasting and Control, Hoboken, NJ, U.S.A: John Wiley and Sons, 2015.

[59] U. o. Waikato, "Weka 3: Data Mining Software in Java," [Online]. Available: http://www.cs.waikato.ac.nz/ml/weka/. [Accessed 07 2019].

[60] Pentaho, "Time Series Analysis and Forecasting with Weka," [Online]. Available: 
http://wiki.pentaho.com/display/DATAMINING/Time+Series+Analysis+and+For ecasting+with+Weka. [Accessed 07 2019].

[61] K. Zou, K. Tuncali and S. G. Silverman, "Correlation and simple linear regression," Radiology, vol. 27, no. 3, pp. 617-628, 06, 2003.

[62] T. Fletcher, "Support vector machines explained," University College London, London, U.K., 2009.

[63] T. Prickett Morgan, "A Rare Peek Into The Massive Scale of AWS," Enterprise Tech, 1411 2014. [Online]. Available: http://www.enterprisetech.com/2014/11/14/rare-peek-massive-scale-aws/. [Accessed 07 2019].

[64] A. Ghodsi, M. Zaharia, S. Shenker and I. Stoica, "Choosy: max-min fair sharing for datacenter jobs with constraints," in Proc. of the 8th ACM European Conference on Computer Systems (EuroSys '13), New York, U.S.A, pp. 365-378, 04, 2013.

[65] A. Shieh, S. Kandula, A. Greenber and C. Kim, "Seawall: performance isolation for cloud datacenter networks," in Proc. of the 2nd USENIX conference on Hot topics in cloud computing, Berkeley, CA, U.S.A, pp. 1-1, 2010.

[66] J. Lee, Y. Turner, M. Lee, L. Popa, S. Banerjee, J.-M. Kang and P. Sharma, "Application-driven bandwidth guarantees in datacenters," in Proc. of the 2014 ACM conference on SIGCOMM, Chicago, U.S.A, pp. 467-478, 2014.

[67] C. Wang, C. Wan and Y. Yuan, "Lease Data Center in the Light of Network Resources: An Economic Model," in Proc. of Fourth International Conference on 
Instrumentation and Measurement, Computer, Communication and Control (IMCCC), Harbin, China, 09, 2014.

[68] J. Yu, R. Buyya and C. K. Tham, " Cost-based scheduling of scientific workflow applications on utility grids," in Proc. of the First International Conference on eScience and Grid Computing, Melbourne, Australia, 07, 2005.

[69] L. Galanis, Y. Wang, S. R. Jeffery and D. J. DeWitt, "Locating data sources in large distributed systems," in Proc. of VLDB '03 Proceedings of the 29th international conference on Very large data bases - Volume 29, Berlin, Germany, pp. 874-885, 09, 2003.

[70] J. Barr, "Per-Second Billing for EC2 Instances and EBS Volumes," Amazon AWS, 1809 2017. [Online]. Available: https://aws.amazon.com/blogs/aws/newper-second-billing-for-ec2-instances-and-ebs-volumes/. [Accessed 2206 2019].

[71] Amazon AWS, "Amazon EC2 Reserved Instances," Amazon, [Online]. Available: https://aws.amazon.com/ec2/pricing/reserved-instances/. [Accessed 06 2019].

[72] Amazon AWS, "Amazon EC2 Reserved Instances Pricing," [Online]. Available: https://aws.amazon.com/ec2/pricing/reserved-instances/pricing/. [Accessed 07 2019].

[73] R. Grossman, "The Case for Cloud Computing," IT Professional, vol. 11, no. 2, pp. 23-27, 032009 .

[74] J. White, B. Dougherty and D. C. Schmidt, "Model-driven auto-scaling of green cloud computing infrastructure," Future Generation Computer Systems, vol. 28, no. 2, pp. 371-378, 02, 2012. 
[75] S. Islam, K. Jacky, L. Kevin and L. Anna, "Empirical prediction models for adaptive resource provisioning in the cloud," Future Generation Computer Systems, vol. 28, no. 1, pp. 155-162, 01, 2012.

[76] D. Kusic , J. O. Kephart, J. E. Hanson, N. Kandasamy and G. Jiang, "Power and performance management of virtualized computing environments via lookahead control," Cluster Computing, vol. 12, no. 1, pp. 1 - 15, 03, 2009.

[77] "JSON," [Online]. Available: http://www.json.org/. [Accessed 1412 2015].

[78] L. M. Vaquero, L. Rodero-Merino and R. Buyya, "Dynamically scaling applications in the cloud," ACM SIGCOMM Computer Communication Review, vol. 41, no. 1, pp. 45-52, 01, 2011.

[79] A. Greenberg, J. R. Hamilton, N. Jain, S. Kandula, C. Kim, P. Lahiri, D. A. Maltz, P. Patel and S. Sengupta, "VL2: a scalable and flexible data center network," $A C M$ SIGCOMM Computer Communication Review, vol. 39, no. 4, pp. pp. 51-62, 10, 2009.

[80] Timothy Prickett Morgan, "A Rare Peek Into The Massive Scale of AWS," 1411 2014. [Online]. Available: http://www.enterprisetech.com/2014/11/14/rare-peekmassive-scale-aws/. [Accessed 12 2015].

[81] Orzell, Greg; Becker, Justin;, "Auto Scaling in the Amazon Cloud," Netflix, 1801 2012. [Online]. Available: http://techblog.netflix.com/2012/01/auto-scaling-inamazon-cloud.html. [Accessed 12 2015].

[82] Irwin, Conrad;, "Autoscaling with Capistrano, NFS and Runit," Bugsnag, 2705 2014. [Online]. Available: https://bugsnag.com/blog/autoscaling-capistrano-nfs- 
runit. [Accessed 12 2015].

[83] A. Biswas, S. Majumdar, B. Nandy and A. El-Haraki, "An Auto-Scaling Framework for Controlling Enterprise Resources on Clouds," in Proc. of 15th IEEE/ACM International Symposium on Cluster, Cloud and Grid Computing (CCGrid), C4BIE Workshop, Shenzhen, China, pp. 971 - 980, 05, 2015.

[84] D. Breitgand, E. Henis and O. Shehory, "Automated and Adaptive Threshold Setting: Enabling Technology for Autonomy and Self-Management," in Proc. of the 2nd International Conference on Automatic Computing (ICAC'05), Seattle, U.S.A, pp. 204-215, 062005.

[85] R. Buyya, C. S. Yeo and S. Venugopal, "Market-Oriented Cloud Computing: Vision, Hype, and Reality for Delivering IT Services as Computing Utilities," in Proc. of the 10th IEEE International Conference on High Performance Computing and Communications (HPCC), Dalian, China, pp 5 - 13, 09, 2008.

[86] M. D. de Assuncao, A. di Costanzo and R. Buyya, "Evaluating the cost-benefit of using cloud computing to extend the capacity of clusters," in Proc. of the 18th ACM international symposium on High performance distributed computing, New York, U.S.A, pp. 141-150, 06, 2009.

[87] M. Mao and M. Humphrey, "A Performance Study on the VM Startup Time in the Cloud," in Proc. of IEEE 5th International Conference on Cloud Computing (CLOUD), Honolulu, HI, U.S.A, pp. 423 - 430, 06, 2012.

[88] M. Mao and M. Humphrey, "Auto-scaling to minimize cost and meet application deadlines in cloud workflows," in Proc. of High Performance Computing, 
Networking, Storage and Analysis, Seatle, WA, U.S.A, pp. 1 - 12, 11, 2011.

[89] N. Roy, A. Dubey and A. Gokhale, "Efficient Autoscaling in the Cloud Using Predictive Models for Workload Forecasting," in Proc. of IEEE International Conference on Cloud Computing (CLOUD), Washington, DC, U.S.A, pp. 500 507, 07, 2011.

[90] W. Wang, B. Li and B. Liang, "Revenue maximization with dynamic auctions in IaaS cloud markets," in Proc. of 21st International Symposium on Quality of Service (IWQoS), Montreal, QC, Canada, pp. 1 - 6, 06, 2013.

[91] J. Yang, C. Liu, Y. Shang and Z. Mao, "Workload Predicting-Based Automatic Scaling in," in Proc. of IEEE Sixth International Conference on Cloud Computing (CLOUD), Santa Clara, CA, U.S.A, pp. 810 - 815, 06, 2013.

[92] I. Foster, C. Kesselman, C. Lee, B. Lindell, C. Nahrstedt and A. Roy, "A distributed resource management architecture that supports advance reservations and co-allocation," in Proc. of Seventh International Workshop on Quality of Service (IWQoS), London, U.K., pp. 27 - 36, 05, 1999.

[93] P. Tang, "Efficient Auto-Scaling Approach in the Telco Cloud Using SelfLearning Algorithm," in IEEE Global Communications Conference (GLOBECOM)., San Diego, CA, USA, 2015.

[94] "Amazon CloudWatch," [Online]. Available: http://aws.amazon.com/cloudwatch/. [Accessed 11 2014].

[95] J. O. Melendez and S. Majumdar, "Matchmaking on Clouds and Grids," J. Internet Techonology, vol. 13, no. 6, pp. 853-866, 112012. 
[96] A. Sulistio and R. Buyya, "A Grid Simulation Infrastructure Supporting Advance Reservation," in Proc. of the 16th International Conference on Parallel and Distributed Computing and Systems, Boston, pp. 1 - 7, 112004.

[97] Apache, "Apache Spark ${ }^{\mathrm{TM}}$ - Unified Analytics Engine for Big Data," [Online]. Available: https://spark.apache.org/. [Accessed 1111 2018].

[98] S.-x. Wang and X.-q. Zhao, "The improved Dijkstra's shortest path algorithm," in Proc. of the Seventh International IEEE Conference on Natural Computation, Shanghai, China, pp. 2313-2316, 07, 2011. 